\title{
Resolution of Nonsingularities of Families of Curves $^{\dagger}$
}

\author{
By \\ Akio TAMAGAWA* \\ On the occasion of the fortieth anniversary of the RIMS
}

\begin{abstract}
In the present paper, we consider the following problem: For a given closed point $x$ of a special fiber of a generically smooth family $X \rightarrow S$ of stable curves (with $\operatorname{dim}(S)=1$ ), is there a covering $Y \rightarrow X$ that is generically étale (i.e., étale over the generic fiber(s) of $X \rightarrow S$, not only over the generic point(s) of $X$ ), where $Y$ is also a family of stable curves, such that the image in $X$ of the non-smooth locus of $Y$ contains $x$ ? Among other things, we prove that this is affirmative (possibly after replacing $S$ by a finite extension) in the case where $S$ is the spectrum of a discrete valuation ring of mixed characteristic whose residue field is algebraic over $\mathbb{F}_{p}$.
\end{abstract}

\section{$\S 0 . \quad$ Introduction}

In algebraic geometry, nonsingular varieties are usually easier to treat than singular varieties. Thus, resolution of singularities — or, equivalently, desingularization - in the sense of modifying singular varieties into nonsingular varieties is a fundamental process in the study of algebraic varieties.

Sometimes, however, singular varieties are easier to treat than nonsingular varieties. One reason for this is that singular varieties are sometimes built up

Communicated by T. Kawai. Received August 16, 2004. Revised August 31, 2004, September 6, 2004.

2000 Mathematics Subject Classification(s): Primary 14H30; Secondary 11G20, 14B05, 14D06, $14 \mathrm{H} 10$

${ }^{\dagger}$ This article is an invited contribution to a special issue of Publications of RIMS commemorating the fortieth anniversary of the founding of the Research Institute for Mathematical Sciences.

* Research Institute for Mathematical Sciences, Kyoto University, Kyoto 606-8502, Japan. e-mail: tamagawa@kurims.kyoto-u.ac.jp 
from more elementary varieties. Moreover, it is sometimes useful to consider nonsingular varieties as deformations of (more elementary) singular varieties. For example, in the arithmetic-geometric study of elliptic curves, to consider the Tate curve is indispensable.

In this sense, we sometimes need to consider "resolution of nonsingularities" — or "singularization" — in the sense of modifying nonsingular varieties (resp. smooth families of varieties) into singular varieties (resp. non-smooth families of varieties). The theme of the present paper is, as is shown in the title, resolution of nonsingularities in this sense, for (mainly 1-dimensional) families of curves.

In the case of resolution of singularities, we allow modifications like normalizations, blowing-ups, alterations ([D1]), and so on. In the present paper, we allow a certain kind of alteration. More precisely, we allow modifying a given (smooth) family of curves by taking a proper, generically finite covering, which is finite étale over the generic fiber of the original family and itself is again a stable family of curves.

Such a situation naturally arises in the arithmetic-geometric study of coverings of curves. Indeed, the technique of resolution of nonsingularities was first introduced to anabelian geometry in [M2], where Mochizuki reduces, by considering certain resolution of nonsingularities, the Grothendieck conjecture for proper, hyperbolic curves over number fields to the Grothendieck conjecture for proper, singular, stable curves over finite fields, which is then reduced to the Grothendieck conjecture for affine - namely, more elementary in the above sense - curves over finite fields [Tam1]. The present paper is partly motivated by this work of Mochizuki.

Now, to state our main problem and main results more precisely, let $S$ be a 1-dimensional, noetherian, normal, integral, separated scheme and denote by $K$ the function field of $S$. We refer to a pair $\left(S^{\prime}, K^{\prime}\right)$ as a finite extension of $(S, K)$, if $K^{\prime}$ is a finite extension of $K$ and $S^{\prime}$ is the integral closure of $S$ in $K$. (For simplicity, we sometimes refer to $S^{\prime}$ as a finite extension of $S$.) Thus, $K^{\prime}$ coincides with the function field of $S^{\prime}$. Let $\left(X^{*}, D\right)$ be a stable marked curve (cf. $§ 1$ ), which is generically smooth in the sense that the generic fiber $X_{K}^{*}$ is smooth over $K$. (By definition, $D_{K}$ is automatically étale over $K$.)

We consider the following conditions (I), (II), (III) and (III').

(I) There exist a finite extension $\left(S^{\prime}, K^{\prime}\right)$ of $(S, K)$ and a generically tame, stable covering (cf. $\S 1)\left(Y_{S^{\prime}}^{*}, E_{S^{\prime}}\right)$ of $\left(X_{S^{\prime}}^{*}, D_{S^{\prime}}\right)$ over $S^{\prime}$, such that $\left(Y_{S^{\prime}}^{*}\right)^{\text {non-sm }} \neq \emptyset$.

(II) For each closed point $s$ of $S$, there exist a finite extension $\left(S^{\prime}, K^{\prime}\right)$ of $(S, K)$ 
and a generically tame, stable covering $\left(Y_{S^{\prime}}^{*}, E_{S^{\prime}}\right)$ of $\left(X_{S^{\prime}}^{*}, D_{S^{\prime}}\right)$ over $S^{\prime}$, such that the image of $\left(Y_{S^{\prime}}^{*}\right)^{\text {non-sm }}$ in $S$ contains $s$.

(III) For each closed point $x$ of $X^{*}$, there exist a finite extension $\left(S^{\prime}, K^{\prime}\right)$ of $(S, K)$ and a generically tame, stable covering $\left(Y_{S^{\prime}}^{*}, E_{S^{\prime}}\right)$ of $\left(X_{S^{\prime}}^{*}, D_{S^{\prime}}\right)$ over $S^{\prime}$, such that the image of $\left(Y_{S^{\prime}}^{*}\right)^{\text {non-sm }}$ in $X^{*}$ contains $x$.

(III') For each closed point $x$ of $X^{*}$, there exist a finite extension $\left(S^{\prime}, K^{\prime}\right)$ of $(S, K)$ and a generically tame, stable covering $\left(Y_{S^{\prime}}^{*}, E_{S^{\prime}}\right)$ of $\left(X_{S^{\prime}}^{*}, D_{S^{\prime}}\right)$ over $S^{\prime}$, such that the image in $X^{*}$ of the vertical components (cf. $\S 2$ ) in the fibers of $Y_{S^{\prime}}^{*} \rightarrow X_{S^{\prime}}^{*}$ contains $x$.

Here, we have used the following notation:

Definition. Let $f: X \rightarrow S$ be a morphism of schemes. We define the smooth locus $X^{\text {sm }}$ of $f$ to be the set of points of $X$ at which $f$ is smooth. Thus, $X^{\mathrm{sm}}$ is an (a possibly empty) open subset of $X$, and we define the closed subset $X^{\text {non-sm }}$ of $X$ to be the complement of $X^{\text {sm }}$ in $X$.

Remark 0.1. (i) Clearly, we have (III') $\Longrightarrow$ (III) $\Longrightarrow$ (II) $\Longrightarrow$ (I). (ii) At first glance, (II) (resp. (III), (III')) appears to be a statement that only concerns a single closed point $s$ of $S$ (resp. $x$ of $X^{*}$ ). However, if we consider the tower of (generically tame, stable) coverings of $\left(X^{*}, D\right)$, then it may be regarded as a statement that concerns all of the closed points of $S$ (resp. $X^{*}$ ). If, moreover, (II) (resp. (III), (III')) holds for every member of the tower of coverings of $\left(X^{*}, D\right)$, then it may be regarded as a statement that concerns all the closed points of all the finite extensions of $S$ (resp. all the coverings of $X^{*}$ ).

The following is a summary of the main results of the present paper. Here, we say that a (non-empty) scheme $S$ is of characteristic 0 (resp. of characteristic $>0$, resp. of mixed characteristic), if the image of $S$ in $\operatorname{Spec}(\mathbb{Z})$ consists of the generic point (resp. a single closed point, resp. more than one point) of $\operatorname{Spec}(\mathbb{Z})$. Moreover, we say that $S$ is strictly of mixed characteristic, if, for each point $t$ of $S$, either $\{t\}^{\mathrm{cl}}$ or $\operatorname{Spec}\left(\mathcal{O}_{S, t}\right)$ is of mixed characteristic. (Throughout this paper, "cl" stands for the topological closure.) When $S$ is 1-dimensional and irreducible, $S$ is of characteristic 0 (resp. of characteristic $>0$, resp. of mixed characteristic, resp. strictly of mixed characteristic), if the inverse image of the generic point of $\operatorname{Spec}(\mathbb{Z})$ in $S$ coincides with the whole of $S$ (resp. is empty, resp. is neither the whole of $S$ nor empty, resp. consists of the generic point of $S)$. 
Theorem 0.2. $\quad$ Let $S$ be a 1-dimensional, noetherian, normal, integral, separated scheme and $\left(X^{*}, D\right)$ a generically smooth, stable marked curve over $S$.

(i) Assume that $S$ is of characteristic 0 and that $\left(X^{*}, D\right)$ is smooth. Then (I) never holds.

(ii) Assume that $S$ is of characteristic $p>0$, that $S$ is proper over a field $k$ (necessarily of characteristic $p$ ), and that $\left(X^{*}, D\right)$ is not $k$-isotrivial (cf. $\left.\S 1\right)$. Then (I) holds. In this case, (II) does not always hold.

(iii) Assume that $S$ is of characteristic $p>0$, that the residue field of each closed point of $S$ is algebraic over $\mathbb{F}_{p}$, and that $\left(X^{*}, D\right)$ is not $\mathbb{F}_{p}$-isotrivial. Then (II) holds. (In this case, the author does not know - and is interested in - whether or not (III) always holds.)

(iv) Assume that $S$ is strictly of mixed characteristic. Then (II) holds. In this case, (III) does not always hold.

(v) Assume that $S$ is strictly of mixed characteristic, and that the residue field of each closed point of $S$ is algebraic over $\mathbb{F}_{p}$ for some $p>0$. Then (III') holds. (In particular, (III) holds.)

Here, (i) and (iv) are widely known, or, at least, can be easily derived from widely known facts. ((iv) essentially appeared in above-mentioned [M2].) (ii) and (iii) are straightforward applications of [Sa] and [Tam3], respectively. ((iii) was used in [St].) (v) is also an application of [Tam3], but requires some extra arguments. Thus, (v) may be regarded as the main new contribution of the present paper.

Remark 0.3. An elliptic modular curve (with a suitable level) gives an example of stable marked curve over (an open subscheme of) the spectrum of the ring of integers of an algebraic number field. In this case, if we only consider coverings corresponding to congruence subgroups of $P S L_{2}(\mathbb{Z})$, then it is classically known (see, e.g., $[\mathrm{KM}]$ ) that supersingular points are the only points whose nonsingularity can be resolved. In particular, there are only finitely many such points in each fiber. On the other hand, if we consider all coverings (possibly corresponding to non-congruence subgroups), Theorem 0.2(v) above says that the nonsingularity of every closed point can be resolved. In this sense, we might regard every closed point as a "non-congruence supersingular point".

We shall explain the content of each $\S$ briefly.

In $\S 1$ and $\S 2$, we give a review of generalities on stable curves and their coverings. The main aim of these $\S$ 's is to fix the definitions and the notations, and there is nothing mathematically new. We use the notion of log structures 
of Fontaine-Illusie-Kato ([Ka]), but in a rather restricted situation that comes from a generically smooth, stable marked curve over the spectrum of a discrete valuation ring.

In $\S 3$, we give a review of three main ingredients of the proofs of the main results. The first one is from [Tam2]. More precisely, we present an average theorem (Theorem 3.1) concerning $p$-ranks of prime-to- $p$-cyclic coverings of hyperbolic curves in characteristic $p>0$. We also prove a generalization (Theorem 3.10) to stable curves under a certain "abelian-injective" assumption. The second one is from [Tam3] and [Sa]. More precisely, we present specialization theorems (Theorem 3.13, Theorem 3.14) to the effect that the specialization map of tame fundamental groups of a family of hyperbolic curves in positive characteristic is not an isomorphism (under suitable assumptions). The third one is from [Tat] and [D2]. More precisely, we present "Tate's theorem" (Theorem 3.16) on $p$-divisible groups. We also apply this to obtain some results (Theorem 3.18, Corollary 3.19) on Galois representations on (étale parts of) Tate modules of abelian varieties.

In $\S 4$, we present various conditions related to the above "resolution of nonsingularities" conditions (I), (II), (III) and (III'). More precisely, in Proposition 4.1, we give various necessary and sufficient conditions for (II), while, in Proposition 4.3, we give various sufficient conditions for (III) and (III'). Most implications are standard and/or straightforward but a few implications are nontrivial (and require Theorem 3.10 and Corollary 3.19).

In $\S 5$, We give a proof of our main Theorem 0.2 . With various results of $\S 3$ and $\S 4$, almost nothing remains for the proof of (i)-(iv). However, as has been mentioned above, the proof of (v) requires some extra arguments.

\section{$\S 1$. Generalities on Stable Curves}

In this $\S$, we review some generalities concerning stable curves.

$\langle$ Hyperbolic and stable marked curves $\rangle$

Let $S$ be a scheme. Let $f^{*}: X^{*} \rightarrow S$ be an $S$-scheme and $D \subset X^{*}$ a closed subscheme. Let $(g, r)$ be a pair of non-negative integers.

Definition. We say that $\left(X^{*}, D\right)$ (or, more precisely, $\left(f^{*}: X^{*} \rightarrow S, D \subset\right.$ $\left.X^{*}\right)$ ) is a smooth marked curve of type $(g, r)$ over $S$, if $f^{*}$ is proper, smooth, the geometric fibers of $f^{*}$ are (automatically proper, smooth) connected curves of genus $g$, and $D$ is finite étale of (constant) degree $r$ over $S$.

Observe that, if $S$ is non-empty, the type $(g, r)$ is completely determined by the smooth marked curve $\left(X^{*}, D\right) / S$. 
Definition. We say that $\left(X^{*}, D\right)$ (or, more precisely, $\left(f^{*}: X^{*} \rightarrow S, D \subset\right.$ $\left.\left.X^{*}\right)\right)$ is a hyperbolic marked curve of type $(g, r)$ over $S$, if it is a smooth marked curve of type $(g, r)$ over $S$ and $\chi\left(\left(X^{*}, D\right) / S\right) \stackrel{\text { def }}{=} 2-2 g-r<0$ holds.

Definition. (i) We say that $\left(X^{*}, D\right)$ (or, more precisely, $\left(f^{*}: X^{*} \rightarrow\right.$ $\left.\left.S, D \subset X^{*}\right)\right)$ is a stable marked curve of type $(g, r)$ over $S$, if $f^{*}$ is proper, flat, its geometric fibers are (automatically proper) connected, reduced curves of arithmetic genus $g$ whose singularities are ordinary double points, $D$ is contained in $X^{\mathrm{sm}}, D$ is finite étale of (constant) degree $r$ over $S$, and the following holds: For each geometric point $\bar{s}$ of $S$, let $D_{\bar{s}}^{\prime}$ denote the (disjoint) union of $D_{\bar{s}}=D \times_{X^{*}} X_{\bar{s}}^{*}$ and $\left(X_{\bar{s}}^{*}\right)^{\text {non-sm }}$ (considered as a reduced closed subscheme of $\left.X_{\bar{s}}^{*}\right)$. Then the normalization $\left(Z^{*}\right)^{\sim}$ of each irreducible component $Z^{*}$ of $X_{\bar{s}}^{*}$, together with $\left.D_{\bar{s}}^{\prime}\right|_{\left(Z^{*}\right)^{\sim}}=D_{\bar{s}}^{\prime} \times_{X_{\bar{s}}^{*}}\left(Z^{*}\right)^{\sim}$, forms a hyperbolic marked curve over $\bar{s}$.

(ii) Assume that $\left(X^{*}, D\right)$ is a stable marked curve over $S$. Then we say that $\left(X^{*}, D\right)$ is generically smooth, if there exists an open dense subscheme $T$ of $S$, such that $\left(X^{*}, D\right) \times_{S} T$ is a smooth marked curve over $T$.

Thus, a hyperbolic marked curve is just a stable marked curve which is also a smooth marked curve. Also, observe that, if $S$ is non-empty, the type $(g, r)$ is completely determined by the stable marked curve $\left(X^{*}, D\right) / S$, and automatically satisfies $\chi\left(\left(X^{*}, D\right) / S\right) \stackrel{\text { def }}{=} 2-2 g-r<0$.

$\langle$ Moduli spaces of stable marked curves $\rangle$

Let $(g, r)$ be a pair of non-negative integers with $2-2 g-r<0$.

Definition. We denote by $\mathcal{M}_{g,[r]}$ (resp. $\overline{\mathcal{M}}_{g,[r]}$ ) the moduli stack over $\mathbb{Z}$ classifying hyperbolic marked curves (resp. stable marked curves) of type $(g, r)$.

$\mathcal{M}_{g,[r]}\left(\operatorname{resp} . \overline{\mathcal{M}}_{g,[r]}\right)$ coincides with the quotient $\left[\mathcal{M}_{g, r} / S_{r}\right]$ (resp. $\left[\overline{\mathcal{M}}_{g, r} /\right.$ $\left.S_{r}\right]$ ) (in the sense of stacks) of the moduli stack $\mathcal{M}_{g, r}\left(\operatorname{resp} . \overline{\mathcal{M}}_{g, r}\right)$ in [Kn] by the natural action of the symmetric group $S_{r}$. Thus, $\mathcal{M}_{g,[r]}$ and $\overline{\mathcal{M}}_{g,[r]}$ form algebraic stacks in the sense of $[\mathrm{DM}] . \overline{\mathcal{M}}_{g,[r]}$ is proper over $\mathbb{Z}$, and $\mathcal{M}_{g,[r]}$ is an open substack of $\overline{\mathcal{M}}_{g,[r]}$. See also [M5], $§ 0$.

Here, the essence of the properness of $\overline{\mathcal{M}}_{g,[r]}$ is the following well-known stable reduction theorem.

Definition. Let $T \rightarrow S$ be a morphism of schemes. Let $\left(X_{T}^{*}, D_{T}\right)$ be a stable marked curve over $T$. Then we say that $\left(X_{T}^{*}, D_{T}\right)$ is defined over (or, sometimes, descends to or extends to) $S$, if there exists a stable marked curve $\left(X^{*}, D\right)$ over $S$, such that $\left(X_{T}^{*}, D_{T}\right)$ is isomorphic to $\left(X^{*}, D\right) \times_{S} T$ over $T$. 
Theorem 1.1. Let $S$ be a 1-dimensional, noetherian, normal, integral, separated scheme and $K$ the function field of $S$. Let $\left(X_{K}^{*}, D_{K}\right)$ be a stable marked curve over $K$. Then there exists a finite separable extension $K^{\prime}$ of $K$, such that $\left(X_{K}^{*}, D_{K}\right) \times{ }_{K} K^{\prime}$ is defined over the integral closure $S^{\prime}$ of $S$ in $K^{\prime}$.

Definition. We denote by $M_{g,[r]}\left(\right.$ resp. $\left.\bar{M}_{g,[r]}\right)$ the coarse moduli space over $\mathbb{Z}$ associated with $\mathcal{M}_{g,[r]}\left(\right.$ resp. $\left.\overline{\mathcal{M}}_{g,[r]}\right)$.

$M_{g,[r]}\left(\right.$ resp. $\left.\bar{M}_{g,[r]}\right)$ is a quasi-projective (resp. projective) scheme over $\mathbb{Z}$, and $M_{g,[r]}$ is an open subscheme of $\bar{M}_{g,[r]}$.

$\langle$ Isotriviality of stable marked curves $\rangle$

Let $k$ be a field, $S$ a connected $k$-scheme, and $\left(X^{*}, D\right)$ a hyperbolic marked curve of type $(g, r)$ over $S$. Although the following is more or less well-known, we include the proof for the sake of completeness, since there appear to be several non-equivalent definitions of isotriviality in the literature.

Proposition 1.2. $\quad$ Consider the following conditions:

(i) There exist a finite extension $k^{\prime}$ of $k, a k^{\prime}$-scheme $S^{\prime}$, and a finite, étale, surjective $k$-morphism $S^{\prime} \rightarrow S$, such that $\left(X^{*}, D\right) \times{ }_{S} S^{\prime}$ descends to $\operatorname{Spec}\left(k^{\prime}\right)$.

(ii) There exist an algebraic extension $k^{\prime}$ of $k, a k^{\prime}$-scheme $S^{\prime}$, and a $k$-morphism $\pi: S^{\prime} \rightarrow S$ with $\mathcal{O}_{S} \rightarrow \pi_{*}\left(\mathcal{O}_{S^{\prime}}\right)$ injective, such that $\left(X^{*}, D\right) \times_{S} S^{\prime}$ descends to $\operatorname{Spec}\left(k^{\prime}\right)$.

(iii) The (set-theoretic) image of the classifying morphism $S \rightarrow M_{g,[r], k}$ over $k$ consists of a single closed point, where $M_{g,[r], k}$ denotes the coarse moduli space over $k$ associated with $\mathcal{M}_{g,[r], k} \stackrel{\text { def }}{=} \mathcal{M}_{g,[r]} \times{ }_{\mathbb{Z}} k$.

(iii') The (set-theoretic) image of the $k$-linearization $S \rightarrow\left(M_{g,[r]}\right)_{k} \stackrel{\text { def }}{=}\left(M_{g,[r]}\right)$ $\times_{\mathbb{Z}} k$ of the classifying morphism $S \rightarrow M_{g,[r]}$ over $\mathbb{Z}$ consists of a single closed point.

Then we have (i) $\Longleftrightarrow$ (ii) $\Longrightarrow$ (iii) $\Longleftrightarrow$ (iii'). If, moreover, $S$ is reduced, all the conditions are equivalent.

Proof. Since a finite, étale, surjective morphism satisfies the injectivity in (ii), we have (i) $\Longrightarrow$ (ii).

Conversely, assume that (ii) holds. By assumption, we have a 1-commutative diagram

$$
\begin{gathered}
S^{\prime} \rightarrow \operatorname{Spec}\left(k^{\prime}\right) \\
\downarrow \quad \quad \downarrow \\
S \rightarrow \mathcal{M}_{g,[r], k} .
\end{gathered}
$$


Take a non-empty, finite, étale covering $M$ of $\mathcal{M}_{g,[r], k}$, such that $M$ is a scheme. By base-changing the above diagram by $M \rightarrow \mathcal{M}_{g,[r], k}$, we obtain another commutative diagram

$$
\begin{array}{ccc}
S_{M}^{\prime} & \rightarrow & \operatorname{Spec}\left(k^{\prime}\right)_{M} \\
\downarrow & \downarrow \\
S_{M} & \rightarrow & M,
\end{array}
$$

which is in the category of $k$-schemes.

As $M \rightarrow \mathcal{M}_{g,[r], k}$ is finite étale, $\operatorname{Spec}\left(k^{\prime}\right)_{M}$ is a finite disjoint union of spectra of finite (separable) extensions of $k^{\prime}$. As $k^{\prime}$ is an algebraic extension of $k$, this implies that the image of $\operatorname{Spec}\left(k^{\prime}\right)_{M} \rightarrow M$ is a finite set $\Sigma$ of closed points of $M$. We define $Z$ to be the disjoint union of spectra of residue fields of points in $\Sigma$. Then $Z$ is a closed subscheme of $M$, and the morphism $\operatorname{Spec}\left(k^{\prime}\right)_{M} \rightarrow M$ factors as $\operatorname{Spec}\left(k^{\prime}\right)_{M} \rightarrow Z \hookrightarrow M$. Moreover, as $M \rightarrow \mathcal{M}_{g,[r], k}$ is flat, the injectivity condition in (ii) is still valid for $S_{M}^{\prime} \rightarrow S_{M}$. It follows immediately from this that the morphism $S_{M} \rightarrow M$ must also factor through $Z$. Take any connected component $S^{\prime \prime}$ of $S_{M}$. Then the natural map $S^{\prime \prime} \rightarrow M$ must factor through $\operatorname{Spec}\left(k^{\prime \prime}\right) \hookrightarrow M$, where $k^{\prime \prime}$ is the residue field of some $z \in \Sigma$.

Note that $k^{\prime \prime}$ is a finite extension of $k$. Moreover, since $S$ is assumed to be connected, $S^{\prime \prime} \rightarrow S$ must be (finite, étale and) surjective. Therefore, the (1-)commutative diagram

$$
\begin{array}{ccc}
S^{\prime \prime} & \rightarrow & \operatorname{Spec}\left(k^{\prime \prime}\right) \\
\downarrow & & \downarrow \\
S_{M} & \rightarrow & M \\
\downarrow & & \downarrow \\
S & \rightarrow & \mathcal{M}_{g,[r], k}
\end{array}
$$

shows that (i) holds. This completes the proof of (ii) $\Longrightarrow$ (i).

Since the natural $k$-morphism $M_{g,[r], k} \rightarrow\left(M_{g,[r]}\right)_{k}$ is finite, radicial (cf. $[\mathrm{KM}]$, Proposition A7.2.1 and Corollary A7.2.2), we have (iii) $\Longleftrightarrow\left(\right.$ iii' $^{\prime}$ ).

Next, again assume that (ii) holds. By assumption, we have a 1-commutative diagram

$$
\begin{aligned}
& S^{\prime} \rightarrow \operatorname{Spec}\left(k^{\prime}\right) \\
& \downarrow \quad \downarrow \\
& S \rightarrow \mathcal{M}_{g,[r], k},
\end{aligned}
$$

which induces a commutative diagram

$$
\begin{gathered}
S^{\prime} \rightarrow \operatorname{Spec}\left(k^{\prime}\right) \\
\downarrow \\
S \rightarrow M_{g,[r], k},
\end{gathered}
$$


in the category of $k$-schemes. Let $z$ be the image of $\operatorname{Spec}\left(k^{\prime}\right) \rightarrow M_{g,[r], k}$, which is a closed point as $k^{\prime}$ is an algebraic extension of $k$. Similarly as in the proof of (ii) $\Longrightarrow$ (i), this implies that $S \rightarrow M_{g,[r], k}$ factors through $\operatorname{Spec}\left(k^{\prime \prime}\right) \hookrightarrow M_{g,[r], k}$, where $k^{\prime \prime}$ is the residue field of $z$. This shows that (iii) holds. This completes the proof of (ii) $\Longrightarrow$ (iii).

Finally, under the extra assumption that $S$ is reduced, suppose that (iii) holds. Let $z$ denote the closed point that appears in the statement of (iii). Then, by the reducedness of $S$, the classifying $k$-morphism $S \rightarrow M_{g,[r], k}$ schemetheoretically factors through $\operatorname{Spec}\left(k^{\prime \prime}\right) \hookrightarrow M_{g,[r], k}$, where $k^{\prime \prime}$ is the residue field of $z$. Take $M$ as in the proof of (ii) $\Longrightarrow$ (i), and set $S_{M} \stackrel{\text { def }}{=} S \times_{\mathcal{M}_{g,[r], k}} M$. Then we have the following 1-commutative diagram:

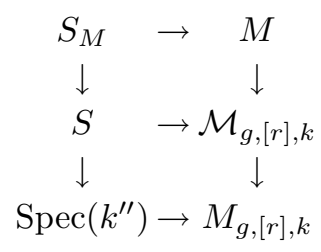

Thus, the morphism $S_{M} \rightarrow M$ factors through the natural closed immersion $\operatorname{Spec}\left(k^{\prime \prime}\right) \times_{M_{g,[r], k}} M \hookrightarrow M$.

Since the composite of $M \rightarrow \mathcal{M}_{g,[r], k} \rightarrow M_{g,[r], k}$ is finite, $\operatorname{Spec}\left(k^{\prime \prime}\right) \times_{M_{g,[r], k}}$ $M \rightarrow M$ is a finite $k^{\prime \prime}$-scheme, hence set-theoretically consists of a finite number of (closed) points. Take any connected component $S^{\prime}$ of $S_{M}$. Then the image of $S^{\prime}$ in $M$, or, equivalently, in $\operatorname{Spec}\left(k^{\prime \prime}\right) \times_{M_{g,[r], k}} M$ must consist of a single closed point $z^{\prime}$ of $M$, at least set-theoretically. Since $S^{\prime}$ is reduced as being étale over the reduced scheme $S$, this implies that $S^{\prime} \rightarrow M$ factors as $S^{\prime} \rightarrow$ $\operatorname{Spec}\left(k^{\prime}\right) \hookrightarrow M$, where $k^{\prime}$ is the residue field of $z^{\prime}$. Thus, $S^{\prime} \rightarrow \mathcal{M}_{g,[r], k}$ factors through $S^{\prime} \rightarrow \operatorname{Spec}\left(k^{\prime}\right)$, a fortiori. Since $S^{\prime} \rightarrow S$ is finite, étale and surjective (as $S$ is connected), this implies that (i) holds.

Thus, the proof is completed.

Definition. We say that $\left(X^{*}, D\right)$ is $k$-isotrivial if either (i) or (ii) (hence both of (i) and (ii)) of Proposition 1.2 holds.

\section{$\S 2$. Coverings of Stable Curves}

In this $\S$, we review some generalities concerning coverings and fundamental groups of stable curves.

〈Structure of local fundamental groups of stable curves $\rangle$ 
Lemma 2.1. Let $R$ be a complete discrete valuation ring and $\mathfrak{m}$ the maximal ideal of $R$. Assume that the residue field $k=R / \mathfrak{m}$ is separably closed (or, equivalently, that $R$ is strictly henselian). Set $p \stackrel{\text { def }}{=} \operatorname{char}(k) \geq 0$. Then:

(i) We have

$$
\pi_{1}(R[[T]])=\{1\}
$$

(ii) We have

$$
\pi_{1}^{\mathrm{t}}\left(\operatorname{Spec}\left(R[[T]]\left[T^{-1}\right]\right)\right) \simeq \widehat{\mathbb{Z}}^{p^{\prime}} \stackrel{\text { def }}{=} \prod_{l \neq p} \mathbb{Z}_{l},
$$

where $\pi_{1}^{\mathrm{t}}\left(\operatorname{Spec}\left(R[[T]]\left[T^{-1}\right]\right)\right)$ denotes the quotient of $\pi_{1}\left(\operatorname{Spec}\left(R[[T]]\left[T^{-1}\right]\right)\right)$ that corresponds to coverings which are at most tamely ramified at the point $(T=0)$ in the generic fiber. Moreover, the "universal (tame) covering" is given by $R[[T]]\left[T^{-1}\right]\left[T^{1 / n} \mid n \geq 1, p \nmid n\right]$.

(iii) Let a be an element of $\mathfrak{m}^{n}-\mathfrak{m}^{n+1}$ for some $n \geq 1$. Then we have

$$
\pi_{1}(\operatorname{Spec}(R[[T, S]] /(T S-a))-\{\mathfrak{M}\}) \simeq \mathbb{Z} / n^{\prime} \mathbb{Z}
$$

where $\mathfrak{M}$ is the maximal ideal of $R[[T, S]] /(T S-a)$, and $n^{\prime}$ is the maximal divisor of $n$ that is not divisible by $p$. Moreover, the "universal covering" is given by $\operatorname{Spec}\left(R\left[\left[T^{\prime}, S^{\prime}\right]\right] /\left(T^{\prime} S^{\prime}-a^{\prime}\right)\right)-\left\{\mathfrak{M}^{\prime}\right\}$, where $T^{\prime}=T^{1 / n^{\prime}}, S^{\prime}=S^{1 / n^{\prime}}$, $a^{\prime}=a^{1 / n^{\prime}}$, and $\mathfrak{M}^{\prime}$ is the maximal ideal of $R\left[\left[T^{\prime}, S^{\prime}\right]\right] /\left(T^{\prime} S^{\prime}-a^{\prime}\right)$.

Proof. (i) As $R[[T]]$ is strictly henselian, all finite étale coverings over $\operatorname{Spec}(R[[T]])$ are trivial.

(ii) [SGA1], Exposé XIII, Corollaire 5.3 (a version of Abhyankar's lemma).

(iii) In the case where $n$ is not divisible by $p$, this can be seen in [M1], $\S 3.12$, Lemma. In general, multiplying $T$ with a unit if necessary, we may assume that $a=\pi^{n}$. Then, by a similar argument as loc. cit., one verifies easily that the universal covering is between $\left.\operatorname{Spec}\left(R\left[\left[T^{\prime}, S^{\prime}\right]\right] /\left(T^{\prime} S^{\prime}-a^{\prime}\right)\right)-\left\{\mathfrak{M}^{\prime}\right\}\right)$ and $\left.\operatorname{Spec}\left(R\left[\left[T^{\prime \prime}, S^{\prime \prime}\right]\right] /\left(T^{\prime \prime} S^{\prime \prime}-a^{\prime \prime}\right)\right)-\left\{\mathfrak{M}^{\prime \prime}\right\}\right)$, where $T^{\prime \prime}=T^{1 / n}, S^{\prime \prime}=S^{1 / n}, a^{\prime \prime}=$ $\pi=a^{1 / n}$, and $\mathfrak{M}^{\prime \prime}$ is the maximal ideal of $R\left[\left[T^{\prime \prime}, S^{\prime \prime}\right]\right] /\left(T^{\prime \prime} S^{\prime \prime}-a^{\prime \prime}\right)$. Now, since $\left.\left.\operatorname{Spec}\left(R\left[\left[T^{\prime \prime}, S^{\prime \prime}\right]\right] /\left(T^{\prime \prime} S^{\prime \prime}-a^{\prime \prime}\right)\right)-\left\{\mathfrak{M}^{\prime \prime}\right\}\right) \rightarrow \operatorname{Spec}\left(R\left[\left[T^{\prime}, S^{\prime}\right]\right] /\left(T^{\prime} S^{\prime}-a^{\prime}\right)\right)-\left\{\mathfrak{M}^{\prime}\right\}\right)$ is totally ramified (with purely inseparable residue field extensions) over the two (generic) points of the special fiber, we can conclude that the universal covering must coincide with $\left.\operatorname{Spec}\left(R\left[\left[T^{\prime}, S^{\prime}\right]\right] /\left(T^{\prime} S^{\prime}-a^{\prime}\right)\right)-\left\{\mathfrak{M}^{\prime}\right\}\right)$.

〈Coverings between stable curves

Let $S$ be a scheme, and $\left(X^{*}, D\right),\left(Y^{*}, E\right)$ stable marked curves over $S$. 
Definition. Let $f: Y^{*} \rightarrow X^{*}$ be a morphism over $S$. Then we denote by $V=V_{Y^{*} / X^{*}}$ the set of points $y$ of $Y$ with $\operatorname{dim}\left(f^{-1}(f(y))\right)=1$, and call it the union of vertical components of $f$. (Observe that $V$ is a closed subset of $Y^{*}$.)

Definition. (i) A stable covering from $\left(Y^{*}, E\right)$ to $\left(X^{*}, D\right)$ over $S$ is a surjective morphism $f: Y^{*} \rightarrow X^{*}$ over $S$ that induces a (unique) (finite, étale) morphism $E \rightarrow D$ and a (unique) morphism $Y^{*}-(E \cup V) \rightarrow X^{*}-D$.

(ii) We say that a stable covering $f:\left(Y^{*}, E\right) \rightarrow\left(X^{*}, D\right)$ over $S$ is finite, if $f$ is finite.

(iii) We say that a finite stable covering $f:\left(Y^{*}, E\right) \rightarrow\left(X^{*}, D\right)$ over $S$ is an admissible covering, if (a) $\left(Y^{*}\right)^{\text {non-sm }}=f^{-1}\left(\left(X^{*}\right)^{\text {non-sm }}\right)$ and $f:\left(Y^{*}\right)^{\text {sm }}-E=$ $f^{-1}\left(\left(X^{*}\right)^{\mathrm{sm}}-D\right) \rightarrow\left(X^{*}\right)^{\mathrm{sm}}-D$ is étale, (b) étale locally at each point of $E$,

$$
\left(Y^{*}, E\right) \stackrel{f}{\rightarrow}\left(X^{*}, D\right) \rightarrow S
$$

is isomorphic to

$$
(\operatorname{Spec}(R[T]),(T=0)) \stackrel{\phi_{n}}{\rightarrow}(\operatorname{Spec}(R[T]),(T=0)) \rightarrow \operatorname{Spec}(R)
$$

for some $n \geq 1, n \in R^{\times}$, where $\phi_{n}$ is induced by the $R$-algebra homomorphism $R[T] \rightarrow R[T], T \mapsto T^{n}$, and (c) étale locally at each point of $\left(Y^{*}\right)^{\text {non-sm }}$,

$$
\left(Y^{*}, E\right) \stackrel{f}{\rightarrow}\left(X^{*}, D\right) \rightarrow S
$$

is isomorphic to

$$
(\operatorname{Spec}(R[S, T] /(S T-b)), \emptyset) \stackrel{\psi_{n}}{\rightarrow}\left(\operatorname{Spec}\left(R[S, T] /\left(S T-b^{n}\right)\right), \emptyset\right) \rightarrow \operatorname{Spec}(R)
$$

for some $n \geq 1, n \in R^{\times}, b \in R$, where $\psi_{n}$ is induced by the $R$-algebra homomorphism $R[S, T] /\left(S T-b^{n}\right) \rightarrow R[S, T] /(S T-b), S \mapsto S^{n}, T \mapsto T^{n}$.

(iv) We say that a finite stable covering $f:\left(Y^{*}, E\right) \rightarrow\left(X^{*}, D\right)$ over $S$ is a tame covering, if $f: Y^{*}-E=f^{-1}\left(X^{*}-D\right) \rightarrow X^{*}-D$ is étale, and the condition of (iii)(b) holds.

(v) We say that a finite stable covering $f:\left(Y^{*}, E\right) \rightarrow\left(X^{*}, D\right)$ over $S$ is an étale covering, if $f: Y^{*} \rightarrow X^{*}$ is étale.

(vi) Let $\mathbf{P}$ be a property of stable coverings. Then we say that a stable covering $f:\left(Y^{*}, E\right) \rightarrow\left(X^{*}, D\right)$ over $S$ is generically $\mathbf{P}$, if there exists an open dense subscheme $T$ of $S$ such that $f_{T}:\left(Y_{T}^{*}, E_{T}\right) \rightarrow\left(X_{T}^{*}, D_{T}\right)$ is $\mathbf{P}$.

The following proposition (which should be widely known) is fundamental. 
Proposition 2.2. $\quad$ Let $S$ be a 1-dimensional, noetherian, normal, integral, separated scheme and $K$ the function field of $S$. Let $\left(X^{*}, D\right),\left(Y^{*}, E\right)$ be generically smooth, stable marked curves over $S$. Let $f_{K}:\left(Y_{K}^{*}, E_{K}\right) \rightarrow$ $\left(X_{K}^{*}, D_{K}\right)$ be a stable covering over $K$. Then:

(i) $f_{K}$ extends uniquely to a stable covering $f:\left(Y^{*}, E\right) \rightarrow\left(X^{*}, D\right)$ over $S$. Moreover, this extension is compatible with any flat base change of 1-dimensional, noetherian, normal, integral, separated schemes.

(ii) Assume, moreover, that $f_{K}$ is a tame covering, and that the degree of the Galois closure $\bar{K}\left(Y_{\bar{K}}^{*}\right)^{\sim} / \bar{K}\left(X_{\bar{K}}^{*}\right)$ of $\bar{K}\left(Y_{\bar{K}}^{*}\right) / \bar{K}\left(X_{\bar{K}}^{*}\right)$ is not divisible by the characteristic of any point of $S$. Then the unique extension $f$ of $f_{K}$ in (i) is an admissible covering.

(iii) Assume, moreover, that $\left(X^{*}, D\right)$ is smooth over $S$, that $f_{K}$ is a tame (resp. an étale) covering, and that the degree of the Galois closure $\bar{K}\left(Y_{\bar{K}}^{*} \sim / \bar{K}\left(X_{\bar{K}}^{*}\right)\right.$ of $\bar{K}\left(Y_{\bar{K}}^{*}\right) / \bar{K}\left(X_{\bar{K}}^{*}\right)$ is not divisible by the characteristic of any point of $S$. Then the unique extension $f$ of $f_{K}$ in (i) is a tame (resp. an étale) covering.

Proof. (i) Various proofs of the first assertion for special cases can be seen in [M2], Lemma 8.3; [M2], Remark after Lemma 8.3, [LL], Proposition 4.4(a) and [LL], Remark 4.6. Similar proofs work well for our general case. Or, we can resort to [M3] or the theory of stable maps (cf. [AO]) (after replacing $S$ by a suitable extension if necessary). The second assertion follows from the uniqueness in the first assertion.

(ii) The proof of this fact for a slightly less general case can be seen in [M1], $\S 3.13$, Lemma. A similar proof works well for our case. Namely, we can resort to Abhyankar's lemma and Lemma 2.1(i)(ii)(iii).

(iii) All the ingredients of the proofs of these facts can be seen in [SGA1], Exposé X and Exposé XIII, respectively. Or, we can resort to Abhyankar's lemma and Lemma 2.1(i)(ii).

\section{〈Various fundamental groups of stable curves〉}

Let $S$ be a scheme, $\left(X^{*}, D\right)$ a stable marked curve over $S$, and set $X=$ $X^{*}-D$.

If $S$ is connected, then $X$ and $X^{*}$ are also connected, since all the fibers are connected. In this case, after choosing suitable geometric points, we obtain the fundamental groups $\pi_{1}(X), \pi_{1}\left(X^{*}\right), \pi_{1}(S)$ and the surjective homomorphisms of profinite groups $\pi_{1}(X) \rightarrow \pi_{1}\left(X^{*}\right) \rightarrow \pi_{1}(S)$. Here, $\pi_{1}\left(X^{*}\right)$ controls the étale coverings of $\left(X^{*}, D\right)$. When $S=\operatorname{Spec}(K)$ with $K$ a field, we have the following 
commutative diagram in which both rows are exact:

$$
\begin{aligned}
& \begin{array}{ccc}
1 \rightarrow \pi_{1}\left(X_{\bar{K}}\right) & \rightarrow \pi_{1}(X) \rightarrow G_{K} \rightarrow 1 \\
\downarrow & \downarrow & \downarrow
\end{array} \\
& 1 \rightarrow \pi_{1}\left(\left(X^{*}\right) \frac{\bar{K}}{K}\right) \rightarrow \pi_{1}\left(X^{*}\right) \rightarrow G_{K} \rightarrow 1,
\end{aligned}
$$

where $G_{K} \stackrel{\text { def }}{=} \operatorname{Gal}\left(K^{\text {sep }} / K\right)=\pi_{1}(\operatorname{Spec}(K))$ is the absolute Galois group of $K$. For more details on fundamental groups, see [SGA1].

Next, if $S$ is connected, then, after choosing a suitable (possibly log) geometric point, we also have the tame fundamental group $\pi_{1}^{\mathrm{t}}(X) \stackrel{\text { def }}{=} \pi_{1}^{\mathrm{t}}\left(X^{*}, D\right)$ that controls the tame coverings of $\left(X^{*}, D\right)$. We have $\pi_{1}(X) \rightarrow \pi_{1}^{\mathrm{t}}(X) \rightarrow$ $\pi_{1}\left(X^{*}\right)$. When $S=\operatorname{Spec}(K)$ with $K$ a field, we have the following exact sequence:

$$
1 \rightarrow \pi_{1}^{\mathrm{t}}\left(X_{\bar{K}}\right) \rightarrow \pi_{1}^{\mathrm{t}}(X) \rightarrow G_{K} \rightarrow 1
$$

For more details on tame fundamental groups, see [SGA1] and [GM].

Finally, let $R$ be a complete discrete valuation ring, and let $K$ and $k$ denote the field of fractions and the residue field, respectively, of $R$. Assume $S=\operatorname{Spec}(R)$ and set $s=\operatorname{Spec}(k)$, which is the closed point of $S . S$ is equipped with a natural $\log$ structure $R-\{0\} \rightarrow R$, and we denote the resulting log scheme by $S^{\log }$. The pull-back of this $\log$ structure to the closed point $s$ yields the $\log$ scheme $s^{\log }$ (an object that is often referred to as a "log point"). Now, assume that $\left(X^{*}, D\right)$ is generically smooth, or, equivalently, $X_{K}^{*}$ is smooth over $\operatorname{Spec}(K)$. Then $X^{*}$ is equipped with a natural $\log$ structure defined by the functions that are invertible outside the divisor $X_{s}^{*} \cup D$ (which may not be a normal crossing divisor in general, though). We denote by the resulting log

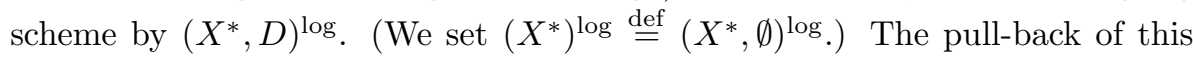
$\log$ structure to the special fiber $X_{s}^{*}$ yields the log scheme $\left(X_{s}^{*}, D_{s}\right)^{\log }$, which is called the log special fiber. Then, after choosing suitable geometric points, we obtain the $\log$ fundamental groups $\pi_{1}^{\log }(S) \stackrel{\text { def }}{=} \pi_{1}\left(S^{\log }\right), \pi_{1}^{\log }(s) \stackrel{\text { def }}{=} \pi_{1}\left(s^{\log }\right)$, $\pi_{1}^{\log }\left(X^{*}, D\right) \stackrel{\text { def }}{=} \pi_{1}\left(\left(X^{*}, D\right)^{\log }\right)$, and $\pi_{1}^{\log }\left(X_{s}^{*}, D_{s}\right) \stackrel{\text { def }}{=} \pi_{1}\left(\left(X_{s}^{*}, D_{s}\right)^{\log }\right)$. Roughly speaking, $\pi_{1}^{\log }\left(X^{*}, D\right)$ controls the admissible coverings of $\left(X^{*}, D\right)$ over finite, at most tamely ramified extensions of $S$. Next, set $S^{\mathrm{t}} \stackrel{\text { def }}{=} \operatorname{Spec}\left(R^{\mathrm{t}}\right)$, where $R^{\mathrm{t}}$ denotes the maximal tamely ramified extension of $R$ (in a fixed algebraic closure of $K)$. Then $S^{\mathrm{t}}$ is equipped with a natural $\log$ structure $R^{\mathrm{t}}-\{0\} \rightarrow R^{\mathrm{t}}$, and we denote the resulting $\log$ scheme by $\left(S^{\mathrm{t}}\right)^{\log }$. The pullback of this $\log$ structure to the closed point $s^{\mathrm{t}}=\operatorname{Spec}\left(k^{\mathrm{sep}}\right)$ of $S^{\mathrm{t}}$ yields the $\log$ scheme $\left(s^{\mathrm{t}}\right)^{\log }$. Writing $S^{\mathrm{t}}$ as a projective limit of the spectra of finite tamely ramified extensions of $R$, one verifies immediately that $X_{S^{t}}^{*}$ is naturally equipped with a structure of $\log$ scheme $\left(X_{S^{t}}^{*}, D_{S^{t}}\right)^{\log }$, which induces a 
structure of log scheme $\left(X_{s^{t}}^{*}, D_{s^{t}}\right)^{\log }$ on $X_{s^{t}}^{*}$. Now, by choosing suitable log geometric points, we obtain the following diagram in which all three rows are exact:

$$
\begin{aligned}
& \begin{array}{cccccc}
1 \rightarrow & \pi_{1}^{\mathrm{t}}\left(X_{\bar{K}}\right) \\
\downarrow & & \pi_{1}^{\mathrm{t}}\left(X_{K}\right) & \rightarrow & G_{K} & \rightarrow 1 \\
& \rightarrow & & \downarrow &
\end{array} \\
& 1 \rightarrow \pi_{1}^{\log }\left(X_{S^{t}}^{*}, D_{S^{t}}\right) \rightarrow \pi_{1}^{\log }\left(X^{*}, D\right) \rightarrow \pi_{1}^{\log }(S) \rightarrow 1 \\
& 1 \rightarrow \pi_{1}^{\log }\left(X_{s^{\mathrm{t}}}^{*}, D_{s^{\mathrm{t}}}\right) \rightarrow \pi_{1}^{\log }\left(X_{s}^{*}, D_{s}\right) \rightarrow \pi_{1}^{\log }(s) \rightarrow 1 .
\end{aligned}
$$

(In fact, in this commutative diagram, the three vertical arrows from the first row to the second row are all surjections, while the three vertical arrows from the third row to the second row are all isomorphisms. For more on this, see the next subsection.)

For more details on log fundamental groups, see $[\mathrm{F}],[\mathrm{FK}],[\mathrm{M} 2],[\mathrm{V}],[\mathrm{I}]$ and $[\mathrm{St}]$.

〈Specialization maps of fundamental groups of stable curves $\rangle$

Let $R$ be a complete discrete valuation ring, and set $S \stackrel{\text { def }}{=} \operatorname{Spec}(R)$. Let $K$ and $k$ denote the field of fractions and the residue field, respectively, of $R$, and set $s \stackrel{\text { def }}{=} \operatorname{Spec}(k)$. We set $p \stackrel{\text { def }}{=} \operatorname{char}(k) \geq 0$. Let $\left(X^{*}, D\right)$ be a generically smooth, stable marked curve.

First, the homomorphism $\pi_{1}\left(X_{s}^{*}\right) \rightarrow \pi_{1}\left(X^{*}\right)$ associated with the natural morphism $X_{s}^{*} \rightarrow X^{*}$ is an isomorphism. Composing the inverse of this isomorphism with the natural homomorphism $\pi_{1}\left(X_{K}^{*}\right) \rightarrow \pi_{1}\left(X^{*}\right)$, we obtain the specialization homomorphism

$$
\operatorname{sp}^{\text {ét }}: \pi_{1}\left(X_{K}^{*}\right) \rightarrow \pi_{1}\left(X_{s}^{*}\right) .
$$

(Precisely speaking, we have to choose a path on $X^{*}$ that connects the image of the geometric point of $X_{K}^{*}$ with that of $X_{s}^{*}$. Accordingly, sp ${ }^{\text {et }}$ is well-defined only up to composition with an inner automorphism.) $\mathrm{sp}^{\text {ét }}$ fits into the following commutative diagram in which both rows are exact:

$$
\begin{aligned}
& 1 \rightarrow \pi_{1}\left(X_{\bar{K}}^{*}\right) \rightarrow \pi_{1}\left(X_{K}^{*}\right) \rightarrow G_{K} \rightarrow 1 \\
& \downarrow \mathrm{sp}^{\text {ét }} \quad \downarrow \mathrm{sp}^{\text {ét }} \downarrow \\
& 1 \rightarrow \pi_{1}\left(X_{\bar{s}}^{*}\right) \rightarrow \pi_{1}\left(X_{s}^{*}\right) \rightarrow G_{k} \rightarrow 1,
\end{aligned}
$$

where $\bar{s}=\operatorname{Spec}(\bar{k})$. Here, the three vertical arrows are all surjective. If, moreover, $X^{*}$ is smooth over $S$, sp ét $^{2} \pi_{1}\left(X_{\bar{K}}^{*}\right) \rightarrow \pi_{1}\left(X_{\bar{s}}^{*}\right)$ induces an isomorphism

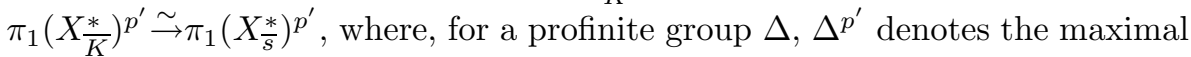
prime-to- $p$ quotient of $\Delta$ (resp. $\Delta$ itself) for $p>0$ (resp. $p=0$ ). 
Second, the homomorphism $\pi_{1}^{\mathrm{t}}\left(X_{s}\right) \rightarrow \pi_{1}^{\mathrm{t}}(X)$ (or, more precisely, $\pi_{1}^{\mathrm{t}}\left(X_{s}^{*}\right.$, $\left.\left.D_{s}\right) \rightarrow \pi_{1}^{\mathrm{t}}\left(X^{*}, D\right)\right)$ associated with the natural morphism $\left(X_{s}^{*}, D_{s}\right) \rightarrow\left(X^{*}, D\right)$ is an isomorphism. Composing the inverse of this isomorphism with the natural homomorphism $\pi_{1}^{\mathrm{t}}\left(X_{K}\right) \rightarrow \pi_{1}^{\mathrm{t}}(X)$, we obtain the specialization homomorphism

$$
\mathrm{sp}^{\mathrm{t}}: \pi_{1}^{\mathrm{t}}\left(X_{K}\right) \rightarrow \pi_{1}^{\mathrm{t}}\left(X_{s}\right) .
$$

(Similarly to the case of étale fundamental groups, we have to choose a path on $\left(X^{*}, D\right)$ that connects the image of the $(\log )$ geometric point of $X_{K}^{*}$ with that of $\left(X_{s}^{*}, D_{s}\right)$. Accordingly, $\mathrm{sp}^{\mathrm{t}}$ is well-defined only up to composition with an inner automorphism.) $\mathrm{sp}^{\mathrm{t}}$ fits into the following commutative diagram in which both rows are exact:

$$
\begin{aligned}
& 1 \rightarrow \pi_{1}^{\mathrm{t}}\left(X_{\bar{K}}\right) \rightarrow \pi_{1}^{\mathrm{t}}\left(X_{K}\right) \rightarrow G_{K} \rightarrow 1 \\
& \downarrow \mathrm{sp}^{\mathrm{t}} \quad \downarrow \mathrm{sp}^{\mathrm{t}} \quad \downarrow \\
& 1 \rightarrow \pi_{1}^{\mathrm{t}}\left(X_{\bar{s}}\right) \rightarrow \pi_{1}^{\mathrm{t}}\left(X_{s}\right) \rightarrow G_{k} \rightarrow 1 \text {. }
\end{aligned}
$$

Here, the three vertical arrows are all surjective. If, moreover, $X^{*}$ is smooth over $S, \mathrm{sp}^{\mathrm{t}}: \pi_{1}^{\mathrm{t}}\left(X_{\bar{K}}\right) \rightarrow \pi_{1}^{\mathrm{t}}\left(X_{\bar{s}}\right)$ induces an isomorphism $\pi_{1}^{\mathrm{t}}\left(X_{\bar{K}}\right)^{p^{\prime}} \stackrel{\sim}{\rightarrow} \pi_{1}$ $\left(X_{\bar{s}}\right)^{p^{\prime}}$.

Third, the homomorphism $\pi_{1}^{\log }\left(X_{s}^{*}, D_{s}\right) \rightarrow \pi_{1}^{\log }\left(X^{*}, D\right)$ associated with the natural morphism $\left(X_{s}^{*}, D_{s}\right)^{\log } \rightarrow\left(X^{*}, D\right)^{\log }$ is an isomorphism. Composing the inverse of this isomorphism with the natural homomorphism $\pi_{1}^{\mathrm{t}}\left(X_{K}\right)=$ $\pi_{1}^{\log }\left(X_{K}^{*}, D_{K}\right) \rightarrow \pi_{1}^{\log }\left(X^{*}, D\right)$, we obtain the specialization homomorphism

$$
\mathrm{sp}^{\log }: \pi_{1}^{\mathrm{t}}\left(X_{K}\right) \rightarrow \pi_{1}^{\log }\left(X_{s}^{*}, D_{s}\right) .
$$

(Similarly to the case of étale and tame fundamental groups, we have to choose a path on $\left(X^{*}, D\right)^{\log }$ that connects the image of the log geometric point of $\left(X_{K}^{*}, D_{K}\right)^{\log }$ with that of $\left(X_{s}^{*}, D_{s}\right)^{\log }$. Accordingly, $\mathrm{sp}^{\log }$ is well-defined only up to composition with an inner automorphism.) $\mathrm{sp}^{\log }$ fits into the following commutative diagram in which both rows are exact:

$$
\begin{aligned}
& 1 \rightarrow \pi_{1}^{\mathrm{t}}\left(X_{\bar{K}}\right) \quad \rightarrow \quad \pi_{1}^{\mathrm{t}}\left(X_{K}\right) \quad \rightarrow \quad G_{K} \rightarrow 1 \\
& \downarrow \mathrm{sp}^{\log } \downarrow \downarrow \mathrm{sp}^{\log } \downarrow \\
& 1 \rightarrow \pi_{1}^{\log }\left(X_{s^{\mathrm{t}}}^{*}, D_{s^{\mathrm{t}}}\right) \rightarrow \pi_{1}^{\log }\left(X_{s}^{*}, D_{s}\right) \rightarrow \pi_{1}^{\log }(s) \rightarrow 1 .
\end{aligned}
$$

Here, the three vertical arrows are all surjective. $\pi_{1}^{\log }(s)$ can be identified with the quotient $G_{K}^{\mathrm{t}} \stackrel{\text { def }}{=} \operatorname{Gal}\left(K^{\mathrm{t}} / K\right)$ of $G_{K}$, where $K^{\mathrm{t}}$ is the field of fractions of $R^{\mathrm{t}}$. Moreover, $\mathrm{sp}^{\log }: \pi_{1}^{\mathrm{t}}\left(X_{\bar{K}}\right) \rightarrow \pi_{1}^{\log }\left(X_{s^{\mathrm{t}}}^{*}, D_{s^{\mathrm{t}}}\right)$ induces an isomorphism

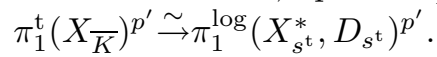


Finally, the above specialization homomorphisms fit into the following commutative diagrams:

$$
\begin{aligned}
& \pi_{1}^{\mathrm{t}}\left(X_{K}^{*}, D_{K}\right)=\pi_{1}^{\mathrm{t}}\left(X_{K}\right) \rightarrow \pi_{1}\left(X_{K}^{*}\right) \\
& \downarrow \mathrm{sp}^{\log } \quad \downarrow \mathrm{sp}^{\mathrm{t}} \quad \downarrow \mathrm{sp}^{\text {ét }} \\
& \pi_{1}^{\log }\left(X_{s}^{*}, D_{s}\right) \rightarrow \pi_{1}^{\mathrm{t}}\left(X_{s}\right) \rightarrow \pi_{1}\left(X_{s}^{*}\right)
\end{aligned}
$$

and

$$
\begin{array}{ccc}
\pi_{1}^{\mathrm{t}}\left(X_{\bar{K}}^{*}, D_{\bar{K}}\right)= & \pi_{1}^{\mathrm{t}}\left(X_{\bar{K}}\right) \rightarrow & \pi_{1}\left(X_{\bar{K}}^{*}\right) \\
\downarrow \mathrm{sp}^{\log } & \downarrow \mathrm{sp}^{\mathrm{t}} & \downarrow \mathrm{sp}^{\text {ét }} \\
\pi_{1}^{\log }\left(X_{s^{\mathrm{t}}}^{*}, D_{s^{\mathrm{t}}}\right) \rightarrow & \pi_{1}^{\mathrm{t}}\left(X_{\bar{s}}\right) \rightarrow & \pi_{1}\left(X_{\bar{s}}^{*}\right) .
\end{array}
$$

Here, we consider the first columns (which involve log fundamental groups) only when $\left(X^{*}, D\right)$ is generically smooth. If, moreover, $\left(X^{*}, D\right)$ is smooth (i.e., $X^{*}$ is smooth over $S)$, then the surjective homomorphism $\pi_{1}^{\log }\left(X_{s^{\mathrm{t}}}^{*}, D_{s^{\mathrm{t}}}\right) \rightarrow \pi_{1}^{\mathrm{t}}\left(X_{\bar{s}}\right)$ in the second diagram is an isomorphism (although $\pi_{1}^{\log }\left(X_{s}^{*}, D_{s}\right) \rightarrow \pi_{1}^{\mathrm{t}}\left(X_{s}\right)$ in the first diagram is not).

$\langle$ Galois representations on fundamental groups of stable curves $\rangle$

Definition. (i) Let $\Delta$ be a profinite group. Then we denote by $\operatorname{Out}(\Delta)$ the outer automorphism group of $\Delta$, i.e., the automorphism group $\operatorname{Aut}(\Delta)$ divided by the inner automorphism group $\operatorname{Inn}(\Delta)$. When $\Delta$ is (topologically) finitely generated, $\operatorname{Out}(\Delta)$ is naturally equipped with a structure of profinite group.

(ii) Let $\Delta_{1}$ and $\Delta_{2}$ be profinite groups, and $\varphi$ a (continuous) homomorphism $\Delta_{1} \rightarrow \Delta_{2}$. Then we set

$$
\begin{aligned}
& \operatorname{Aut}(\varphi) \stackrel{\text { def }}{=}\left\{\left(\alpha_{1}, \alpha_{2}\right) \in \operatorname{Aut}\left(\Delta_{1}\right) \times \operatorname{Aut}\left(\Delta_{2}\right) \mid \varphi \circ \alpha_{1}=\alpha_{2} \circ \varphi\right\}, \\
& \operatorname{Inn}(\varphi) \stackrel{\text { def }}{=} \operatorname{Aut}(\varphi) \cap\left(\operatorname{Inn}\left(\Delta_{1}\right) \times \operatorname{Inn}\left(\Delta_{2}\right)\right),
\end{aligned}
$$

and

$$
\operatorname{Out}(\varphi) \stackrel{\text { def }}{=} \operatorname{Aut}(\varphi) / \operatorname{Inn}(\varphi)\left(\hookrightarrow \operatorname{Out}\left(\Delta_{1}\right) \times \operatorname{Out}\left(\Delta_{2}\right)\right) .
$$

We denote by $\operatorname{pr}_{i}$ the natural projection $\operatorname{Out}(\varphi) \rightarrow \operatorname{Out}\left(\Delta_{i}\right)$ for each $i=1,2$.

Remark 2.3. (i) If $\varphi$ is surjective, then we have

$$
\begin{aligned}
\operatorname{Aut}(\varphi) \stackrel{\sim}{\rightarrow}\left\{\alpha_{1} \in \operatorname{Aut}\left(\Delta_{1}\right)\right. & \left.\mid \alpha_{1}(\operatorname{Ker}(\varphi))=\operatorname{Ker}(\varphi)\right\} \subset \operatorname{Aut}\left(\Delta_{1}\right), \\
& \operatorname{Inn}(\varphi) \stackrel{\sim}{\rightarrow} \operatorname{Inn}\left(\Delta_{1}\right),
\end{aligned}
$$

and

$$
\operatorname{Out}(\varphi) \stackrel{\mathrm{pr}_{1}}{\hookrightarrow} \operatorname{Out}\left(\Delta_{1}\right)
$$


(ii) If $\varphi$ is injective, then we have

$$
\begin{aligned}
\operatorname{Aut}(\varphi) & \stackrel{\sim}{\rightarrow}\left\{\alpha_{2} \in \operatorname{Aut}\left(\Delta_{2}\right) \mid \alpha_{2}(\operatorname{Im}(\varphi))=\operatorname{Im}(\varphi)\right\} \subset \operatorname{Aut}\left(\Delta_{2}\right), \\
\operatorname{Inn}(\varphi) & \stackrel{\sim}{\rightarrow}\left\{\operatorname{Inn}\left(\delta_{2}\right) \mid \delta_{2} \in \operatorname{Im}(\varphi) \cdot C_{\Delta_{2}}(\operatorname{Im}(\varphi))\right\} \subset \operatorname{Inn}\left(\Delta_{2}\right),
\end{aligned}
$$

where $C$ denotes the centralizer, and a natural exact sequence

$$
1 \rightarrow N_{\Delta_{2}}(\operatorname{Im}(\varphi)) /\left(\operatorname{Im}(\varphi) \cdot C_{\Delta_{2}}(\operatorname{Im}(\varphi))\right) \rightarrow \operatorname{Out}(\varphi) \stackrel{\operatorname{pr}_{2}}{\rightarrow} \operatorname{Out}\left(\Delta_{2}\right)
$$

where $N$ denotes the normalizer.

The following is well-known or easy.

Lemma 2.4. (i) Assume that we are given the following exact sequence of profinite groups:

$$
1 \rightarrow \Delta \rightarrow \Pi \rightarrow \Gamma \rightarrow 1 .
$$

Then there exists a unique homomorphism (called an outer representation) $\rho=$ $\rho_{\Pi}: \Gamma \rightarrow \operatorname{Out}(\Delta)$ that fits into the following commutative diagram (in which both rows are exact):

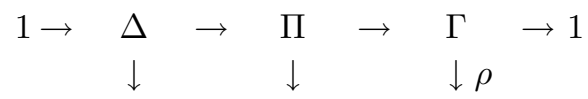

$$
\begin{aligned}
& 1 \rightarrow \operatorname{Inn}(\Delta) \rightarrow \operatorname{Aut}(\Delta) \rightarrow \operatorname{Out}(\Delta) \rightarrow 1,
\end{aligned}
$$

where $\Pi \rightarrow \operatorname{Aut}(\Delta)$ (resp. $\Delta \rightarrow \operatorname{Inn}(\Delta))$ is given by $\left.\pi \mapsto \operatorname{Inn}(\pi)\right|_{\Delta}$ (resp. $\delta \mapsto \operatorname{Inn}(\delta))$. If, moreover, $\Delta$ is finitely generated, then $\rho$ is a (continuous) homomorphism of profinite groups.

(ii) Assume that we are given the following commutative diagram in which both rows are exact:

$$
\begin{aligned}
& 1 \rightarrow \Delta_{1} \rightarrow \Pi_{1} \rightarrow \Gamma_{1} \rightarrow 1 \\
& \downarrow \varphi \downarrow \downarrow \downarrow \\
& 1 \rightarrow \Delta_{2} \rightarrow \Pi_{2} \rightarrow \Gamma_{2} \rightarrow 1 \text {, }
\end{aligned}
$$

and set $\rho_{i} \stackrel{\text { def }}{=} \rho_{\Pi_{i}}$ for $i=1,2$. Then $\rho_{1}$ and $\rho_{2}$ are compatible in the sense that there exists a (unique) homomorphism $\rho_{12}: \Gamma_{1} \rightarrow \operatorname{Out}(\varphi)$ that fits into the following commutative diagram:

$$
\begin{aligned}
& \Gamma_{1}=\Gamma_{1} \rightarrow \Gamma_{2} \\
& \downarrow \rho_{1} \quad \downarrow \rho_{12} \quad \downarrow \rho_{2} \\
& \operatorname{Out}\left(\Delta_{1}\right) \stackrel{\mathrm{pr}_{1}}{\leftarrow} \operatorname{Out}(\varphi) \stackrel{\mathrm{pr}_{2}}{\rightarrow} \operatorname{Out}\left(\Delta_{2}\right) \text {. }
\end{aligned}
$$


Now, let $K$ be a field, and $\left(X^{*}, D\right)$ a stable marked curve over $\operatorname{Spec}(K)$. Then, by the preceding arguments, we obtain outer Galois representations

$$
\rho_{K}^{\text {ét }}=\rho_{\pi_{1}\left(X^{*}\right)}: G_{K} \rightarrow \operatorname{Out}\left(\pi_{1}\left(X_{\bar{K}}^{*}\right)\right)
$$

and

$$
\rho_{K}^{\mathrm{t}}=\rho_{\pi_{1}^{\mathrm{t}}(X)}: G_{K} \rightarrow \operatorname{Out}\left(\pi_{1}^{\mathrm{t}}\left(X_{\bar{K}}\right)\right),
$$

which are compatible in the sense of Lemma 2.4(ii), with respect to the natural surjection $\pi_{1}^{\mathrm{t}}\left(X_{\bar{K}}\right) \rightarrow \pi_{1}\left(X_{\bar{K}}^{*}\right)$.

Next, let $R$ be a complete discrete valuation ring, and set $S \stackrel{\text { def }}{=} \operatorname{Spec}(R)$. Let $K$ and $k$ denote the field of fractions and the residue field, respectively, of $R$, and set $s \stackrel{\text { def }}{=} \operatorname{Spec}(k)$. Let $\left(X^{*}, D\right)$ be a generically smooth, stable marked curve over $S$. Then we obtain the following outer Galois representations:

$$
\begin{gathered}
\rho_{K}^{\text {ét }}=\rho_{\pi_{1}\left(X_{K}^{*}\right)}: G_{K} \rightarrow \operatorname{Out}\left(\pi_{1}\left(X_{\bar{K}}^{*}\right)\right), \\
\rho_{K}^{\mathrm{t}}=\rho_{\pi_{1}^{\mathrm{t}}\left(X_{K}\right)}: G_{K} \rightarrow \operatorname{Out}\left(\pi_{1}^{\mathrm{t}}\left(X_{\bar{K}}\right)\right), \\
\rho_{s}^{\mathrm{et}}=\rho_{\pi_{1}\left(X_{s}^{*}\right)}: G_{k} \rightarrow \operatorname{Out}\left(\pi_{1}\left(X_{\bar{k}}^{*}\right)\right), \\
\rho_{s}^{\mathrm{t}}=\rho_{\pi_{1}^{\mathrm{t}}\left(X_{s}\right)}: G_{k} \rightarrow \operatorname{Out}\left(\pi_{1}^{\mathrm{t}}\left(X_{\bar{k}}\right)\right),
\end{gathered}
$$

and

$$
\rho_{s}^{\log }=\rho_{\pi_{1}^{\log }\left(X_{s}^{*}, D_{s}\right)}: \pi_{1}^{\log }(s) \rightarrow \operatorname{Out}\left(\pi_{1}^{\log }\left(X_{s^{t}}^{*}, D_{s^{t}}\right)\right) .
$$

Various pairs of these homomorphisms are compatible in the sense of Lemma 2.4(ii).

$\langle$ Abelianizations of fundamental groups

Let $K$ be a field, and $\left(X^{*}, D\right)$ a smooth marked curve over $\operatorname{Spec}(K)$. Then we have

$$
\pi_{1}\left(X_{\bar{K}}^{*}\right)^{\mathrm{ab}} \simeq T(J(\bar{K}))
$$

as $G_{K}$-modules, where $J$ denotes the Jacobian variety of $X^{*}$, and, for an abelian group $M$,

$$
T(M) \stackrel{\text { def }}{=} \prod_{l: \text { prime }} T_{l}(M), T_{l}(M) \stackrel{\text { def }}{=} \underbrace{\lim }_{r} \operatorname{Ker}\left(l^{r} \cdot \operatorname{id}_{M}\right) .
$$

Moreover, we have the following exact sequence of $G_{K}$-modules:

$$
0 \rightarrow \widehat{\mathbb{Z}}^{\prime}(1)^{1-b^{(2)}} \rightarrow \mathbb{Z}[D(\bar{K})] \otimes \widehat{\mathbb{Z}}^{\prime}(1) \rightarrow \pi_{1}^{\mathrm{t}}\left(X_{\bar{K}}\right)^{\mathrm{ab}} \rightarrow \pi_{1}\left(X_{\bar{K}}^{*}\right)^{\mathrm{ab}} \rightarrow 0,
$$

where $\widehat{\mathbb{Z}}^{\prime}(1) \stackrel{\text { def }}{=} T\left(\mathbb{G}_{m}(\bar{K})\right)$ and $b^{(2)}=0\left(\right.$ resp. $\left.b^{(2)}=1\right)$ if $D \neq \emptyset($ resp. $D=\emptyset)$. 
Next, let $R$ be a complete discrete valuation ring, and set $S \stackrel{\text { def }}{=} \operatorname{Spec}(R)$. Let $K$ and $k$ denote the field of fractions and the residue field, respectively, of $R$, and set $s \stackrel{\text { def }}{=} \operatorname{Spec}(k)$. Let $\left(X^{*}, D\right)$ be a generically smooth, stable marked curve over $S$. Let $\mathcal{V}$ be the set of irreducible components of $X_{\bar{S}}^{*}$ and set $\mathcal{E} \stackrel{\text { def }}{=}$ $\left(X_{\bar{s}}^{*}\right)^{\text {non-sm }}$. Let $\mathcal{G}=\mathcal{G}_{X_{s}^{*}}$ be the dual graph of $X_{\bar{s}}^{*}$. By definition, the vertex set and the edge set of $\mathcal{G}$ are $\mathcal{V}$ and $\mathcal{E}$, respectively. Observe that $G_{k}$ naturally acts on $\mathcal{G}$, hence, in particular, on $\mathcal{V}$ and on $\mathcal{E}$. For each $v \in \mathcal{V}$, we shall denote by $Z^{*}(v)$ the irreducible component of $X_{\bar{s}}$ corresponding to $v$. (Logically speaking, we have $v=Z^{*}(v)$, though.) Set $Z(v) \stackrel{\text { def }}{=} Z^{*}(v)-\left\{D_{\bar{s}} \cup\left(X_{\bar{s}}^{*}\right)^{\text {non-sm }}\right\}$, which is a smooth curve over $\bar{s}$. Then it is widely known that $\pi_{1}^{\log }\left(X_{s^{t}}^{*}, D_{s^{t}}\right)$ can be described in terms of a certain graph of groups $\mathcal{G G}=\mathcal{G G}_{\left(X_{s^{t}}^{*}, D_{s^{t}}\right)}$, whose graph is just $\mathcal{G}_{X_{s}^{*}}$ and whose vertex group at $v \in \mathcal{V}$ is $\pi_{1}^{\mathrm{t}}(Z(v))$. In particular, we have the following exact sequence of $\pi_{1}^{\log }(s)$-modules:

$$
\underset{v \in \mathcal{V}}{\oplus} \pi_{1}^{\mathrm{t}}(Z(v))^{\mathrm{ab}} \rightarrow \pi_{1}^{\log }\left(X_{s^{\mathrm{t}}}^{*}, D_{s^{\mathrm{t}}}\right)^{\mathrm{ab}} \rightarrow H_{1}(\mathcal{G}, \mathbb{Z}) \otimes \widehat{\mathbb{Z}} \rightarrow 0
$$

where $\pi_{1}^{\log }(s)$ acts on the first and the third terms via its quotient $\pi_{1}(s)=G_{k}$.

$\langle p$-rank and $p$-defect $\rangle$

Let $l$ be a prime number.

Let $K$ be a field, and $A$ a semi-abelian variety over $K$. Thus, $A$ fits into an exact sequence $0 \rightarrow T \rightarrow A \rightarrow B \rightarrow 0$, where $B$ (resp. $T$ ) is an abelian variety (resp. a torus) over $K$. We set

$$
\gamma_{l}(A) \stackrel{\text { def }}{=} \operatorname{rk}_{\mathbb{Z}_{l}}\left(\operatorname{Hom}_{\bar{K}}\left(\mathbb{G}_{m}\left[l^{\infty}\right]_{\bar{K}}, A\left[l^{\infty}\right]_{\bar{K}}\right)\right) .
$$

This is a non-negative integer, and we call it the $l$-rank of $A$. Thus, we have

$$
\gamma_{l}(A)=\operatorname{rk}_{\mathbb{Z}_{l}}\left(T_{l}(A(\bar{K}))\right)=2 \operatorname{dim}(B)+\operatorname{dim}(T)=\operatorname{dim}(A)+\operatorname{dim}(B)
$$

for $l \neq \operatorname{char}(K)$. On the other hand, we have

$$
\begin{aligned}
\gamma_{p}(A) & =\operatorname{rk}_{\mathbb{Z}_{p}}\left(T_{p}(B(\bar{K}))\right)+\operatorname{dim}(T) \\
& \leq \operatorname{dim}(B)+\operatorname{dim}(T)=\operatorname{dim}(A)
\end{aligned}
$$

if $p \stackrel{\text { def }}{=} \operatorname{char}(K)>0$. Here, the equality follows from the fact that an extension of a multiplicative $p$-divisible group by a multiplicative $p$-divisible group is again multiplicative, while the inequality follows from a well-known property of $p$-rank of abelian varieties.

Next, let $R$ be a discrete valuation ring, and let $K$ and $k$ denote the field of fractions and the residue field, respectively, of $R$. Let $A$ be a semi-abelian 
scheme over $R$. Then we set

$$
\delta_{l}(A) \stackrel{\text { def }}{=} \gamma_{l}\left(A_{K}\right)-\gamma_{l}\left(A_{k}\right) .
$$

This is a non-negative integer, and we call it the $l$-defect of $A$. Assume, moreover, that $A$ is generically proper, i.e., that the generic fiber $A_{K}$ is an abelian variety over $K$. As $A_{k}$ is a semi-abelian variety over $k$, it fits into an exact sequence

$$
0 \rightarrow T_{k} \rightarrow A_{k} \rightarrow B_{k} \rightarrow 0
$$

with $B_{k}$ (resp. $T_{k}$ ) an abelian variety (resp. a torus) over $k$. Then we have

$$
\delta_{l}(A)=\operatorname{dim}\left(T_{k}\right)
$$

for $l \neq \operatorname{char}(k)$, and

$$
\delta_{p}(A)=\operatorname{rk}_{\mathbb{Z}_{p}}\left(T_{p}\left(A_{K}(\bar{K})\right)\right)-\left\{\operatorname{rk}_{\mathbb{Z}_{p}}\left(T_{p}\left(B_{k}(\bar{k})\right)\right)+\operatorname{dim}\left(T_{k}\right)\right\}
$$

if $p \stackrel{\text { def }}{=} \operatorname{char}(k)>0$. Moreover, in the latter case, we have

$$
\begin{cases}\operatorname{dim}\left(A_{k}\right) \leq \delta_{p}(A) \leq \operatorname{dim}\left(A_{k}\right)+\operatorname{dim}\left(B_{k}\right), & \operatorname{char}(K)=0, \\ 0 \leq \delta_{p}(A) \leq \operatorname{dim}\left(B_{k}\right), & \operatorname{char}(K)=p .\end{cases}
$$

For a proper, generically smooth, semi-stable curve $X^{*}$ over $R$, let $J_{K}$ be the Jacobian variety of $X_{K}^{*}$ and $J$ the Néron model over $R$ of $J_{K}$. Moreover, let $J^{0}$ denote the group subscheme of $J$ obtained from $J$ by removing all the connected components of $J_{k}$ but the one containing the origin. Then $J^{0}$ is a generically proper, semi-abelian scheme over $R$, and we set $\delta_{l}\left(X^{*}\right) \stackrel{\text { def }}{=} \delta_{l}\left(J^{0}\right)$. Now, by various results of the last subsection and so on, it is not difficult to see that we have

$$
\gamma_{l}\left(\left(J^{0}\right)_{K}\right)=\gamma_{l}\left(J_{K}\right)=\gamma_{l}\left(X_{K}^{*}\right) \stackrel{\text { def }}{=} \operatorname{rk}_{\mathbb{Z}_{l}}\left(\pi_{1}\left(X_{K}^{*}\right)^{\mathrm{ab}} \otimes_{\widehat{\mathbb{Z}}} \mathbb{Z}_{l}\right)
$$

and

$$
\gamma_{l}\left(\left(J^{0}\right)_{k}\right)=\gamma_{l}\left(X_{k}^{*}\right) \stackrel{\text { def }}{=} \operatorname{rk}_{\mathbb{Z}_{l}}\left(\pi_{1}\left(X_{\bar{k}}^{*}\right)^{\mathrm{ab}} \otimes_{\widehat{\mathbb{Z}}} \mathbb{Z}_{l}\right),
$$

hence

$$
\delta_{l}\left(X^{*}\right)=\operatorname{rk}_{\mathbb{Z}_{l}}\left(\pi_{1}\left(X_{\bar{K}}^{*}\right)^{\mathrm{ab}} \otimes_{\widehat{\mathbb{Z}}} \mathbb{Z}_{l}\right)-\operatorname{rk}_{\mathbb{Z}_{l}}\left(\pi_{1}\left(X_{\bar{k}}^{*}\right)^{\mathrm{ab}} \otimes_{\widehat{\mathbb{Z}}} \mathbb{Z}_{l}\right) .
$$

Remark 2.8. It is widely known that $J^{0}$ is also obtained as a certain open subscheme of the Picard scheme of $X$ over $R$. From this description, it follows that the following exact sequence exists:

$$
0 \rightarrow T_{k} \rightarrow\left(J^{0}\right)_{k} \rightarrow B_{k} \rightarrow 0 .
$$


Here, the abelian variety $B_{k}$ is the direct product of the Jacobian varieties of the normalizations of the irreducible components of $X_{k}^{*}$, while the torus $T_{k}$ is of dimension equal to $\operatorname{rk}_{\mathbb{Z}}\left(H_{1}(\mathcal{G}, \mathbb{Z})\right)$.

\section{§3. Main Ingredients of Proof}

In this $\S$, we present three main ingredients of the proofs in $\S 4$ and in $\S 5$.

〈Average theorems on generalized Hasse-Witt invariants〉

We shall start with the following purely group-theoretic definition.

Definition. Let $\Pi$ be a profinite group. Let $N$ be a natural number and $l$ a prime number.

(i) We denote by $\Pi(N)$ the kernel of $\Pi \rightarrow \Pi^{\mathrm{ab}} /\left(\Pi^{\mathrm{ab}}\right)^{N}=\Pi^{\mathrm{ab}} \otimes(\mathbb{Z} / N \mathbb{Z})$. Equivalently, $\Pi(N)$ is the topological closure of the subgroup $[\Pi, \Pi] \Pi^{N}$ of $\Pi$. (ii) We set $\gamma_{l}(\Pi) \stackrel{\text { def }}{=} \operatorname{dim}_{\mathbb{F}_{l}}(\Pi / \Pi(l)) \in \mathbb{Z}_{\geq 0} \cup\{\infty\}$.

(iii) Let $N$ be a natural number such that $(\Pi: \Pi(N))<\infty$. Then we set $\gamma_{l}^{\text {av }}(N)(\Pi) \stackrel{\text { def }}{=} \gamma_{l}(\Pi(N)) /(\Pi: \Pi(N)) \in \mathbb{Q}_{\geq 0} \cup\{\infty\}$. ("av" stands for "average" (over $(\mathbb{Z} / N \mathbb{Z})$-coverings). See [Tam2], Lemma (4.5) and Remark (4.8).)

Let $K$ be an algebraically closed field of characteristic $p \geq 0$. Let $\left(X^{*}, D\right)$ be a hyperbolic marked curve of type $(g, r)$ over $K$, and set $X \stackrel{\text { def }}{=} X^{*}-D$. We set

$$
g^{\prime} \stackrel{\text { def }}{=} \begin{cases}g-1, & r \leq 1 \\ g, & r>1\end{cases}
$$

Observe that we have

$$
\gamma_{l}\left(\pi_{1}^{\mathrm{t}}\left(X_{\bar{K}}\right)\right)= \begin{cases}2 g+r-1+b^{(2)}=\gamma_{l}\left(X_{K}^{*}\right)+r-1+b^{(2)}, & l \neq p, \\ \gamma_{p}\left(X_{K}^{*}\right), & l=p(>0) .\end{cases}
$$

Now, for each natural number $N$, we set

$$
\gamma_{l}^{\text {av }}(N)(X) \stackrel{\text { def }}{=} \gamma_{l}^{\text {av }}(N)\left(X^{*}, D\right) \stackrel{\text { def }}{=} \gamma_{l}^{\text {av }}(N)\left(\pi_{1}^{\mathrm{t}}\left(X_{\bar{K}}\right)\right) .
$$

Then we have:

Theorem 3.1 ([Tam2], Theorem (0.5)). Assume $p>0$. Then we have

$$
\lim _{f \rightarrow \infty} \gamma_{p}^{\mathrm{av}}\left(p^{f}-1\right)(X)=g^{\prime}
$$


Remark 3.2. Let $l$ be a prime number. If $K$ is a field of characteristic $p \neq l$, we have

$$
\lim _{\substack{N \rightarrow \infty \\ p \nmid N}} \gamma_{l}^{\mathrm{av}}(N)\left(\pi_{1}^{\mathrm{t}}\left(X_{\bar{K}}\right)\right)=2 g+r-2 .
$$

This follows essentially from Hurwitz' formula.

We shall generalize this theorem to the non-smooth case. Thus, let $R$ be a complete discrete valuation ring, and set $S \stackrel{\text { def }}{=} \operatorname{Spec}(R)$. Let $K$ and $k$ denote the field of fractions and the residue field, respectively, of $R$, and set $s \stackrel{\text { def }}{=} \operatorname{Spec}(k)$. Set $p \stackrel{\text { def }}{=} \operatorname{char}(k) \geq 0$. Let $\left(X^{*}, D\right)$ be a generically smooth, stable marked curve of type $(g, r)$ over $S$. Let $\mathcal{V}$ be the set of irreducible components of $X_{\bar{s}}^{*}$ and set $\mathcal{E} \stackrel{\text { def }}{=}\left(X_{\bar{s}}^{*}\right)^{\text {non-sm }}$. Let $\mathcal{G}=\mathcal{G}_{X_{s}^{*}}$, the dual graph of $X_{\bar{s}}^{*}$ (cf. $\S 2$ ). By definition, the vertex set and the edge set of $\mathcal{G}$ are $\mathcal{V}$ and $\mathcal{E}$, respectively. Next, we define another graph $\mathcal{G}^{*}=\mathcal{G}_{\left(X_{s}^{*}, D_{\bar{s}}\right)}^{*}$ as follows. If $r=0$, i.e., $D_{\bar{s}}=\emptyset$, we set $\mathcal{G}^{*} \stackrel{\text { def }}{=} \mathcal{G}$. Otherwise, the vertex set of $\mathcal{G}^{*}$ is $\mathcal{V}^{*} \stackrel{\text { def }}{=} \mathcal{V} \amalg\left\{v_{\infty}\right\}$, the edge set of $\mathcal{G}^{*}$ is $\mathcal{E}^{*} \stackrel{\text { def }}{=} \mathcal{E} \coprod D_{\bar{s}}$, and each edge $e \in D_{\bar{s}} \subset \mathcal{E}^{*}$ connects $v_{\infty}$ with the irreducible component that contains the marked point $e \in D_{\bar{s}}$. (Essentially speaking, this is the one-point compactification of the semi-graph associated with $X_{\bar{s}}^{*}-D_{\bar{s}}$ in the sense of [M4], Appendix.) For each $v \in \mathcal{V}$ (resp. $v \in \mathcal{V}^{*}$ ), we set

$$
\nu(v) \stackrel{\text { def }}{=} \sum_{e \in \mathcal{E}} \nu_{e}(v)\left(\text { resp. } \nu^{*}(v) \stackrel{\text { def }}{=} \sum_{e \in \mathcal{E}^{*}} \nu_{e}(v)\right)
$$

where $\nu_{e}(v) \in\{0,1,2\}$ denotes the number of times that $e$ meets $v$.

For each $v \in \mathcal{V}$, let $Z^{*}(v)$ denote the irreducible component of $X_{\bar{s}}^{*}$ corresponding to $v$, set $Z(v) \stackrel{\text { def }}{=} Z^{*}(v)-\left\{D_{\bar{s}} \cup\left(X_{\bar{s}}^{*}\right)^{\text {non-sm }}\right\}$, and define $\left(Z^{*}\right)^{\sim}(v)$ to be the normalization of $Z^{*}(v)$.

Definition. (i) Let $v$ be an element of $\mathcal{V}$. Then we say that $\left(X_{s}^{*}, D_{s}\right)^{\log }$ is abelian-injective at $v$, if the natural homomorphism

$$
\pi_{1}^{\mathrm{t}}(Z(v))^{\mathrm{ab}} \rightarrow \pi_{1}^{\log }\left(X_{s^{\mathrm{t}}}^{*}, D_{s^{\mathrm{t}}}\right)^{\mathrm{ab}}
$$

is injective.

(ii) We say that $\left(X_{s}^{*}, D_{s}\right)^{\log }$ is abelian-injective, if it is abelian-injective at $v$ for each $v \in \mathcal{V}$.

Remark 3.3. One can prove that the natural homomorphism

$$
\pi_{1}^{\mathrm{t}}(Z(v)) \rightarrow \pi_{1}^{\log }\left(X_{s^{\mathrm{t}}}^{*}, D_{s^{\mathrm{t}}}\right)
$$

(well-defined up to inner automorphism) itself is always injective. See [M2], Proposition 4.2 and [St], Proposition 6.2.11. 
Proposition 3.4. For each $v \in \mathcal{V}$, the following conditions are all equivalent.

(i) $\left(X_{s}^{*}, D_{s}\right)^{\log }$ is abelian-injective at $v$.

(i') For each natural number $N$, the natural homomorphism

$$
\pi_{1}^{\mathrm{t}}(Z(v))^{\mathrm{ab}} \otimes \mathbb{Z} / N \mathbb{Z} \rightarrow \pi_{1}^{\log }\left(X_{s^{\mathrm{t}}}^{*}, D_{s^{\mathrm{t}}}\right)^{\mathrm{ab}} \otimes \mathbb{Z} / N \mathbb{Z}
$$

is injective.

(ii) The graph $\mathcal{G}^{*}$ is 2-connected at $v$, i.e., $\mathcal{G}^{*}-\{v\}$ is (either empty or) connected.

Proof. This can be proved by means of the well-known description of $\pi_{1}^{\log }\left(X_{s^{\mathrm{t}}}^{*}, D_{s^{\mathrm{t}}}\right)$ in terms of the graph of groups $\mathcal{G G} \stackrel{\text { def }}{=} \mathcal{G} \mathcal{G}_{\left(X_{s^{\mathrm{t}}}^{*}, D_{s^{\mathrm{t}}}\right)}(\mathrm{cf} . \S 2)$.

$\left(\mathrm{i}^{\prime}\right) \Longrightarrow(\mathrm{i})$. Just take the projective limit (with respect to $N$ ) of the homomorphisms appearing in $\left(\mathrm{i}^{\prime}\right)$.

(i) $\Longrightarrow$ (ii). Suppose that $\mathcal{G}^{*}$ is not 2-connected at $v$, i.e., that $\mathcal{G}^{*}-\{v\}$ is not connected. Then we may choose a connected component $C$ of $\mathcal{G}^{*}-\{v\}$ that does not contain $v_{\infty}$. Let $\nu(C)$ denote the total number of times that the edges contained in $C v \stackrel{\text { def }}{=} C \cup\{v\}$ meet $v$. Let $l$ be a prime number $\neq p$. Then, by means of the fact that $\mathcal{G}^{*}-\{v\}$ has a connected component distinct from $C$, one verifies easily that the rank of the $\mathbb{Z}_{l}$-module $M \subset \pi_{1}^{\mathrm{t}}(Z(v))^{\mathrm{ab}} \otimes_{\widehat{\mathbb{Z}}} \mathbb{Z}_{l}$ generated by the images of the edge groups for the edges contained in $C v$ that meet $v$ is $\nu(C)$. On the other hand, by means of the fact that $C$ does not contain $v_{\infty}$, one verifies easily that the rank of the image of $M$ in $\pi_{1}^{\log }\left(X_{s^{t}}^{*}, D_{s^{t}}\right)^{\mathrm{ab}} \otimes_{\widehat{\mathbb{Z}}} \mathbb{Z}_{l}$ is at most (in fact, just) $\nu(C)-1$. Thus, the map

$$
\pi_{1}^{\mathrm{t}}(Z(v))^{\mathrm{ab}} \otimes_{\widehat{\mathbb{Z}}} \mathbb{Z}_{l} \rightarrow \pi_{1}^{\log }\left(X_{s^{\mathrm{t}}}^{*}, D_{s^{\mathrm{t}}}\right)^{\mathrm{ab}} \otimes_{\widehat{\mathbb{Z}}} \mathbb{Z}_{l}
$$

cannot be injective, and neither can the map,

$$
\pi_{1}^{\mathrm{t}}(Z(v))^{\mathrm{ab}} \rightarrow \pi_{1}^{\log }\left(X_{s^{\mathrm{t}}}^{*}, D_{s^{\mathrm{t}}}\right)^{\mathrm{ab}},
$$

a fortiori.

(ii) $\Longrightarrow\left(\mathrm{i}^{\prime}\right)$. First, note that the map

$$
\pi_{1}\left(\left(Z^{*}\right)^{\sim}(v)\right)^{\mathrm{ab}} \otimes \mathbb{Z} / N \mathbb{Z} \rightarrow \pi_{1}\left(X_{\bar{s}}^{*}\right)^{\mathrm{ab}} \otimes \mathbb{Z} / N \mathbb{Z}
$$

is always injective. For example, this can be proved by means of the description of $\pi_{1}^{\log }\left(X_{s^{t}}^{*}, D_{s^{t}}\right)^{\mathrm{ab}}$ via $\mathcal{G G}$. More precisely, by considering $\pi_{1}^{\log }\left(X_{s^{t}}^{*}, D_{s^{t}}\right)^{\mathrm{ab}}$ modulo all the edge groups, we deduce that $\pi_{1}\left(\left(Z^{*}\right) \sim(v)\right)^{\text {ab }}$ is a direct summand of $\pi_{1}\left(X_{\bar{s}}^{*}\right)^{\text {ab }}$. For each $w \in \mathcal{V}$, let $M_{w}$ be the $(\mathbb{Z} / N \mathbb{Z})$-module generated by the 
images of all the edge groups (i.e., inertia groups) in $\pi_{1}^{\mathrm{t}}(Z(w)) \otimes \mathbb{Z} / N \mathbb{Z}$. Thus, it suffices to prove that $M_{v}$ is mapped injectively into $\pi_{1}^{\log }\left(X_{s^{t}}^{*}, D_{s^{t}}\right)^{\text {ab }} \otimes \mathbb{Z} / N \mathbb{Z}$. In particular, we may assume that $N$ is not divisible by $p$. (Note that, in this case, $M_{v}$ is a free $(\mathbb{Z} / N \mathbb{Z})$-module of rank $\nu^{*}(v)-1$, unless $D=\emptyset$ and $X^{*}$ is smooth.)

For each edge $e \in \mathcal{E}^{*}$, let $M_{e}$ denote the edge group of $\mathcal{G G}$ at $e$ (resp. the inertia group at $e$ ) tensored with $\mathbb{Z} / N \mathbb{Z}$, if $e \in \mathcal{E}$ (resp. $e \in D_{\bar{s}}$ ). (Note that $M_{e}$ is a free $(\mathbb{Z} / N \mathbb{Z}$ )-module of rank 1.) Choose an orientation (among the two possibilities) of each edge once for all. For each pair $(e, w) \in \mathcal{E}^{*} \times \mathcal{V}$, we define $j_{e, w}^{\mathrm{s}}\left(\right.$ resp. $\left.j_{e, w}^{\mathrm{t}}\right): M_{e} \rightarrow M_{w}$ to be the natural map if $w$ is the source (resp. target) of $e$, and the zero map if $w$ is not the source (resp. target) of $e$. (More precisely, the former case is divided into two cases: $e \in \mathcal{E}$ and $e \in D \bar{s}$. For $e \in \mathcal{E}$, we obtain the natural map $M_{e} \rightarrow M_{w}$ by the very definition of the graph of groups $\mathcal{G G}$, while, for $e \in D_{\bar{s}}$, the natural map $M_{e} \rightarrow M_{w}$ is induced by the natural inclusion from the inertia group at $e$ into the vertex group at $w$.) We set $\delta_{e, w} \stackrel{\text { def }}{=} j_{e, w}^{\mathrm{s}}-j_{e, w}^{\mathrm{t}}$.

For each subset $\mathcal{V}^{\prime}\left(\right.$ resp. $\left.\mathcal{E}^{\prime}\right)$ of $\mathcal{V}\left(\right.$ resp. $\left.\mathcal{E}^{*}\right)$, we define $M_{\mathcal{V}^{\prime}}\left(\right.$ resp. $\left.M_{\mathcal{E}^{\prime}}\right)$ to be the direct sum of $M_{w}$ (resp. $M_{e}$ ) for $w \in \mathcal{V}^{\prime}$ (resp. $e \in \mathcal{E}^{\prime}$ ). Thus, we obtain natural maps $j_{\mathcal{E}^{\prime}, \mathcal{V}^{\prime}}^{\mathrm{s}}, j_{\mathcal{E}^{\prime}, \mathcal{V}^{\prime}}^{\mathrm{t}}$, and $\delta_{\mathcal{E}^{\prime}, \mathcal{V}^{\prime}}$ from $M_{\mathcal{E}^{\prime}}$ to $M_{\mathcal{V}^{\prime}}$ as the direct sums of $j_{e, w}^{\mathrm{s}}, j_{e, w}^{\mathrm{t}}$, and $\delta_{e, w}$, respectively, for $(e, w) \in \mathcal{E}^{\prime} \times \mathcal{V}^{\prime}$.

For each (not necessarily connected) subgraph $\mathcal{G}_{1}=\left(\mathcal{V}_{1}, \mathcal{E}_{1}\right)$ of $\mathcal{G}=(\mathcal{V}, \mathcal{E})$, we define $M_{\mathcal{G}_{1}}$ to be the cokernel of $\delta_{\mathcal{E}_{1}, \mathcal{V}_{1}}: M_{\mathcal{E}_{1}} \rightarrow M_{\mathcal{V}_{1}}$. (Thus, one may think of $M_{\mathcal{G}_{1}}$ as the image of $M_{\mathcal{V}_{1}}$, via the natural map, in the direct sum of the abelianizations tensored with $\mathbb{Z} / N \mathbb{Z}$ of the fundamental groups of the connected components of the graph of groups obtained by restricting $\mathcal{G G}$ to $\mathcal{G}_{1}$.) In particular, we obtain a natural inclusion

$$
M_{\mathcal{G}} \hookrightarrow \pi_{1}^{\log }\left(X_{s^{t}}^{*}, D_{s^{t}}\right)^{\mathrm{ab}} \otimes \mathbb{Z} / N \mathbb{Z},
$$

by means of the description of $\pi_{1}^{\log }\left(X_{s^{t}}^{*}, D_{s^{t}}\right)$ via $\mathcal{G G}$.

For each subset $\mathcal{V}^{\prime}$ of $\mathcal{V}$ and each $e \in \mathcal{E}^{*}$, we set

$$
\nu_{e}\left(\mathcal{V}^{\prime}\right) \stackrel{\text { def }}{=} \sum_{w \in \mathcal{V}^{\prime}} \nu_{e}(w) \quad(\in\{0,1,2\}),
$$

and, for each $i \in\{0,1,2\}$,

$$
\mathcal{E}\left(\mathcal{V}^{\prime}, i\right) \stackrel{\text { def }}{=}\left\{e \in \mathcal{E} \mid \nu_{e}\left(\mathcal{V}^{\prime}\right)=i\right\}
$$

and

$$
\mathcal{E}^{*}\left(\mathcal{V}^{\prime}, i\right) \stackrel{\text { def }}{=}\left\{e \in \mathcal{E}^{*} \mid \nu_{e}\left(\mathcal{V}^{\prime}\right)=i\right\} .
$$


Then one verifies immediately

$$
\mathcal{E}=\coprod_{i=0}^{2} \mathcal{E}\left(\mathcal{V}^{\prime}, i\right), \mathcal{E}^{*}=\coprod_{i=0}^{2} \mathcal{E}^{*}\left(\mathcal{V}^{\prime}, i\right),
$$

and $\mathcal{E}\left(\mathcal{V}^{\prime}, 2\right)=\mathcal{E}^{*}\left(\mathcal{V}^{\prime}, 2\right)\left(\right.$ since $\left.\mathcal{V}^{\prime} \subset \mathcal{V}\right)$.

For each subset $\mathcal{V}^{\prime}$ of $\mathcal{V}$, let $\mathcal{G}_{\mathcal{V}^{\prime}}$ denote the "full" subgraph $\left(\mathcal{V}^{\prime}, \mathcal{E}\left(\mathcal{V}^{\prime}, 2\right)\right)$ of $\mathcal{G}$. We denote the natural surjection $M_{\mathcal{V}^{\prime}} \rightarrow M_{\mathcal{G}_{\mathcal{V}^{\prime}}}$ by $p_{\mathcal{V}^{\prime}}$. Now, we claim:

Claim 3.5. Let $\mathcal{V}^{\prime}$ be a subset of $\mathcal{V}$, and assume that $\mathcal{G}_{\mathcal{V}^{\prime}}$ is connected. Let $\mathcal{E}^{\prime}$ be a proper subset of $\mathcal{E}^{*}\left(\mathcal{V}^{\prime}, 1\right)$ (i.e., $\left.\mathcal{E}^{\prime} \varsubsetneqq \mathcal{E}^{*}\left(\mathcal{V}^{\prime}, 1\right)\right)$. Then $p_{\mathcal{V}^{\prime}} \circ \delta_{\mathcal{E}^{\prime}, \mathcal{V}^{\prime}}$ : $M_{\mathcal{E}^{\prime}} \rightarrow M_{\mathcal{G}_{\mathcal{V}^{\prime}}}$ is split-injective, i.e., induces an isomorphism onto a direct summand.

Proof. Although this can be proved by considering a smoothing of the stable marked curve corresponding to $\mathcal{G}_{\mathcal{V}^{\prime}}$, here we shall give a combinatorial proof by induction on $\sharp\left(\mathcal{V}^{\prime}\right)$.

First, assume $\sharp\left(\mathcal{V}^{\prime}\right)=1$, and write $\mathcal{V}^{\prime}=\{w\}$. We define $\bar{M}_{\mathcal{G}_{\mathcal{V}^{\prime}}}$ to be the cokernel of

$$
\left(j_{\mathcal{E}\left(\mathcal{V}^{\prime}, 2\right), \mathcal{V}^{\prime}}^{\mathrm{s}} j_{\mathcal{E}\left(\mathcal{V}^{\prime}, 2\right), \mathcal{V}^{\prime}}^{\mathrm{t}}\right): M_{\mathcal{E}\left(\mathcal{V}^{\prime}, 2\right)} \oplus M_{\mathcal{E}\left(\mathcal{V}^{\prime}, 2\right)} \rightarrow M_{\mathcal{V}^{\prime}}
$$

This is a quotient of $M_{\mathcal{G}_{\mathcal{V}^{\prime}}}$. It follows immediately from the definition of $\bar{M}_{\mathcal{G}_{\mathcal{V}^{\prime}}}$ that there is a natural injection

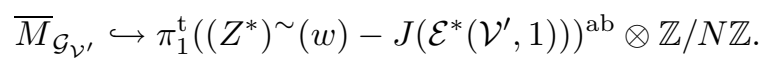

Here, for each $e \in \mathcal{E}^{*}\left(\mathcal{V}^{\prime}, 1\right)$, we define $J(e) \in\left(Z^{*}\right)^{\sim}(w)$ to be the corresponding singular or marked point. Now, it is easy to see that $M_{\mathcal{E}^{\prime}} \rightarrow \bar{M}_{\mathcal{G}_{\mathcal{V}^{\prime}}}$ is splitinjective, hence so is $M_{\mathcal{E}^{\prime}} \rightarrow M_{\mathcal{G}_{\mathcal{V}^{\prime}}}$, a fortiori.

Next, assume $\sharp\left(\mathcal{V}^{\prime}\right)>1$. Then there exists a decomposition $\mathcal{V}^{\prime}=\mathcal{V}_{1}^{\prime} \amalg \mathcal{V}_{2}^{\prime}$, such that $\mathcal{V}_{1}^{\prime} \neq \emptyset, \mathcal{V}_{2}^{\prime} \neq \emptyset$, and that both $\mathcal{G}_{\mathcal{V}_{1}^{\prime}}$ and $\mathcal{G}_{\mathcal{V}_{2}^{\prime}}$ are connected. (For example, choose $w \in \mathcal{V}^{\prime}$ and a connected component $C$ of $\mathcal{G}_{\mathcal{V}^{\prime}}-\{w\}$, define $\mathcal{V}_{1}^{\prime}$ to be the set of vertices that belong to $C$, and set $\mathcal{V}_{2}^{\prime} \stackrel{\text { def }}{=} \mathcal{V}^{\prime}-\mathcal{V}_{1}^{\prime}$.) Let $\mathcal{E}\left[\mathcal{V}_{1}^{\prime}, \mathcal{V}_{2}^{\prime}\right]$ be the set of edges $e \in \mathcal{E}$ that meet both $\mathcal{V}_{1}^{\prime}$ and $\mathcal{V}_{2}^{\prime}$. Then it is easy to see that the following four decompositions exist:

$$
\begin{aligned}
\mathcal{E}^{*}\left(\mathcal{V}_{1}^{\prime}, 1\right) & =\left(\mathcal{E}^{*}\left(\mathcal{V}^{\prime}, 1\right) \cap \mathcal{E}^{*}\left(\mathcal{V}_{1}^{\prime}, 1\right)\right) \coprod \mathcal{E}\left[\mathcal{V}_{1}^{\prime}, \mathcal{V}_{2}^{\prime}\right] \\
\mathcal{E}^{*}\left(\mathcal{V}_{2}^{\prime}, 1\right) & =\left(\mathcal{E}^{*}\left(\mathcal{V}^{\prime}, 1\right) \cap \mathcal{E}^{*}\left(\mathcal{V}_{2}^{\prime}, 1\right)\right) \coprod \mathcal{E}\left[\mathcal{V}_{1}^{\prime}, \mathcal{V}_{2}^{\prime}\right] \\
\mathcal{E}\left(\mathcal{V}^{\prime}, 2\right) & =\mathcal{E}\left(\mathcal{V}_{1}^{\prime}, 2\right) \coprod \mathcal{E}\left(\mathcal{V}_{2}^{\prime}, 2\right) \coprod \mathcal{E}\left[\mathcal{V}_{1}^{\prime}, \mathcal{V}_{2}^{\prime}\right] \\
\mathcal{E}^{*}\left(\mathcal{V}^{\prime}, 1\right) & =\left(\mathcal{E}^{*}\left(\mathcal{V}^{\prime}, 1\right) \cap \mathcal{E}^{*}\left(\mathcal{V}_{1}^{\prime}, 1\right)\right) \coprod\left(\mathcal{E}^{*}\left(\mathcal{V}^{\prime}, 1\right) \cap \mathcal{E}^{*}\left(\mathcal{V}_{2}^{\prime}, 1\right)\right)
\end{aligned}
$$


The last decomposition induces a decomposition $\mathcal{E}^{\prime}=\mathcal{E}_{1}^{\prime} \amalg \mathcal{E}_{2}^{\prime}$, where

$$
\mathcal{E}_{i}^{\prime} \stackrel{\text { def }}{=} \mathcal{E}^{\prime} \cap\left(\mathcal{E}^{*}\left(\mathcal{V}^{\prime}, 1\right) \cap \mathcal{E}^{*}\left(\mathcal{V}_{i}^{\prime}, 1\right)\right)
$$

for $i=1,2$. Since $\mathcal{E}^{\prime} \subsetneq \mathcal{E}^{*}\left(\mathcal{V}^{\prime}, 1\right)$, we have either $\mathcal{E}_{1}^{\prime} \subsetneq \mathcal{E}^{*}\left(\mathcal{V}^{\prime}, 1\right) \cap \mathcal{E}^{*}\left(\mathcal{V}_{1}^{\prime}, 1\right)$ or $\mathcal{E}_{2}^{\prime} \subsetneq \mathcal{E}^{*}\left(\mathcal{V}^{\prime}, 1\right) \cap \mathcal{E}^{*}\left(\mathcal{V}_{2}^{\prime}, 1\right)$. Without loss of generality, we may assume that $\mathcal{E}_{1}^{\prime} \subsetneq \mathcal{E}^{*}\left(\mathcal{V}^{\prime}, 1\right) \cap \mathcal{E}^{*}\left(\mathcal{V}_{1}^{\prime}, 1\right)$ holds. Thus, $\mathcal{E}_{1}^{\prime} \amalg \mathcal{E}\left[\mathcal{V}_{1}^{\prime}, \mathcal{V}_{2}^{\prime}\right] \subsetneq \mathcal{E}^{*}\left(\mathcal{V}_{1}^{\prime}, 1\right)$ also holds. On the other hand, since $\mathcal{V}_{1}^{\prime} \neq \emptyset, \mathcal{V}_{2}^{\prime} \neq \emptyset$, and $\mathcal{G}_{\mathcal{V}^{\prime}}$ is connected, we have $\mathcal{E}\left[\mathcal{V}_{1}^{\prime}, \mathcal{V}_{2}^{\prime}\right] \neq \emptyset$. Thus, we have $\mathcal{E}_{2}^{\prime} \subsetneq \mathcal{E}_{2}^{\prime} \amalg \mathcal{E}\left[\mathcal{V}_{1}^{\prime}, \mathcal{V}_{2}^{\prime}\right] \subset \mathcal{E}^{*}\left(\mathcal{V}_{2}^{\prime}, 1\right)$.

Now, by the induction hypothesis, the natural maps

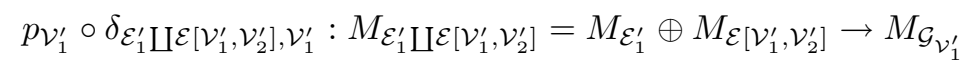

and

$$
p_{\mathcal{V}_{2}^{\prime}} \circ \delta_{\mathcal{E}_{2}^{\prime}, \mathcal{V}_{2}^{\prime}}: M_{\mathcal{E}_{2}^{\prime}} \rightarrow M_{\mathcal{G}_{\mathcal{V}_{2}^{\prime}}}
$$

are split-injective. Thus, we may write

$$
M_{\mathcal{G}_{\mathcal{V}_{1}^{\prime}}}=M_{\mathcal{E}_{1}^{\prime}} \oplus M_{\mathcal{E}\left[\mathcal{V}_{1}^{\prime}, \mathcal{V}_{2}^{\prime}\right]} \oplus N_{1}
$$

for some $N_{1} \subset M_{\mathcal{G}_{\mathcal{V}_{1}^{\prime}}}$, and

$$
M_{\mathcal{G}_{\mathcal{V}_{2}^{\prime}}}=M_{\mathcal{E}_{2}^{\prime}} \oplus N_{2}
$$

for some $N_{2} \subset M_{\mathcal{G}_{\mathcal{V}_{2}^{\prime}}}$. It is immediate from the definition of $M_{\mathcal{G}_{\mathcal{V}^{\prime}}}$ that $M_{\mathcal{G}_{\mathcal{V}^{\prime}}}$ may be identified with the cokernel of the composite of the following:

$$
M_{\mathcal{E}\left[\mathcal{V}_{1}, \mathcal{V}_{2}\right]} \stackrel{\delta_{\mathcal{E}}\left[\mathcal{V}_{1}, \mathcal{V}_{2}\right], \mathcal{V}^{\prime}}{\longrightarrow} M_{\mathcal{V}^{\prime}}=M_{\mathcal{V}_{1}^{\prime}} \oplus M_{\mathcal{V}_{2}^{\prime}} \stackrel{p_{\mathcal{V}_{1}^{\prime}} \oplus p_{\mathcal{V}_{2}^{\prime}}^{\longrightarrow}}{\rightarrow} M_{\mathcal{G}_{\mathcal{V}_{1}^{\prime}}} \oplus M_{\mathcal{G}_{\mathcal{V}_{2}^{\prime}}}
$$

From these observations, we obtain (in light of the various identifications made so far):

$$
\begin{aligned}
M_{\mathcal{G}_{\mathcal{V}^{\prime}}} & =M_{\mathcal{G}_{\mathcal{V}_{1}^{\prime}}} \coprod_{M_{\mathcal{E}\left[\mathcal{V}_{1}^{\prime}, \mathcal{V}_{2}^{\prime}\right]}} M_{\mathcal{G}_{\mathcal{V}_{2}^{\prime}}} \\
& =\left(M_{\mathcal{E}_{1}^{\prime}} \oplus M_{\mathcal{E}\left[\mathcal{V}_{1}^{\prime}, \mathcal{V}_{2}^{\prime}\right]} \oplus N_{1}\right) \coprod_{M_{\mathcal{E}_{\left[\mathcal{V}_{1}^{\prime}, \mathcal{V}_{2}^{\prime}\right]}}} M_{\mathcal{G}_{\mathcal{V}_{2}^{\prime}}} \\
& \leftarrow\left(M_{\mathcal{E}_{1}^{\prime}} \oplus N_{1}\right) \oplus M_{\mathcal{G}_{\mathcal{V}_{2}^{\prime}}} \\
& =\left(M_{\mathcal{E}_{1}^{\prime}} \oplus N_{1}\right) \oplus\left(M_{\mathcal{E}_{2}^{\prime}} \oplus N_{2}\right) .
\end{aligned}
$$

(Here, $A \coprod B$ denotes the amalgamated sum — or, equivalently, fiber coproduct — of $A$ and $B$ with respect to $C$.) Thus, the natural map $M_{\mathcal{E}^{\prime}}=M_{\mathcal{E}_{1}^{\prime}} \oplus M_{\mathcal{E}_{2}^{\prime}} \rightarrow$ $M_{\mathcal{G}_{\mathcal{V}^{\prime}}}$ is split-injective, as desired. 
We shall continue to use the notations of the proof of Claim 3.5. Let $\mathcal{C}$ be the set of connected components of $\mathcal{G}-\{v\}$, and, for each $C \in \mathcal{C}$, let $\mathcal{V}_{C}$ denote the set of vertices in $\mathcal{V}$ that belong to $C$. Then one verifies immediately that $\mathcal{E}^{*}\left(\mathcal{V}_{C}, 1\right)=\mathcal{E}\left[\mathcal{V}_{C},\{v\}\right] \amalg \mathcal{E}^{*}\left[\mathcal{V}_{C},\left\{v_{\infty}\right\}\right]$. Here, for two subsets $\mathcal{V}_{1}^{\prime}$ and $\mathcal{V}_{2}^{\prime}$ of $\mathcal{V}^{*}$ that are disjoint from each other, $\mathcal{E}\left[\mathcal{V}_{1}^{\prime}, \mathcal{V}_{2}^{\prime}\right]$ (resp. $\left.\mathcal{E}^{*}\left[\mathcal{V}_{1}^{\prime}, \mathcal{V}_{2}^{\prime}\right]\right)$ denotes the set of edges in $\mathcal{E}$ (resp. $\mathcal{E}^{*}$ ) that meet both $\mathcal{V}_{1}^{\prime}$ and $\mathcal{V}_{2}^{\prime}$.

Now, assume first that $r\left(=\sharp\left(D_{\bar{s}}\right)\right)>0$. Since $\mathcal{G}^{*}-\{v\}$ is connected by assumption, $\mathcal{E}^{*}\left[\mathcal{V}_{C},\left\{v_{\infty}\right\}\right] \neq \emptyset$ holds for each $C \in \mathcal{C}$. Or, equivalently, $\mathcal{E}\left[\mathcal{V}_{C},\{v\}\right] \subsetneq \mathcal{E}^{*}\left(\mathcal{V}_{C}, 1\right)$ holds for each $C \in \mathcal{C}$. Now, by Claim 3.5, we may write $M_{\mathcal{G}_{\mathcal{V}_{C}}}=M_{\mathcal{E}\left[\mathcal{V}_{C},\{v\}\right]} \oplus N_{C}$ for some $N_{C} \subset M_{\mathcal{G}_{\mathcal{V}_{C}}}$. Thus, we obtain:

$$
\begin{aligned}
M_{\mathcal{G}} & =M_{\mathcal{G}_{\{v\}}} \coprod_{M_{\mathcal{E}[\mathcal{V}-\{v\},\{v\}]}} M_{\mathcal{G}_{\mathcal{V}-\{v\}}}(\mathrm{cf.}(3.6)) \\
& =M_{\mathcal{G}_{\{v\}}} \coprod_{\left(\underset{C \in \mathcal{C}}{\oplus} M_{\mathcal{E}\left[\mathcal{V}_{C},\{v\}\right]}\right)}\left(\underset{C \in \mathcal{C}}{\oplus} M_{\mathcal{G}_{\mathcal{V}_{C}}}\right) \\
& \sim M_{\mathcal{G}_{\{v\}}} \oplus\left(\underset{C \in \mathcal{C}}{\oplus} N_{C}\right) .
\end{aligned}
$$

Note that $Z^{*}(v)=\left(Z^{*}\right) \sim(v)$ holds, since $\mathcal{G}^{*}-\{v\}$ is connected and $r>0$. Thus, the natural surjection $M_{v}=M_{\{v\}} \rightarrow M_{\mathcal{G}_{\{v\}}}$ is an isomorphism. Therefore, the natural map $M_{v} \rightarrow M_{\mathcal{G}}$ is (split-)injective, as desired.

Next, assume $r=0$. If, moreover, $\mathcal{V}=\{v\}$ (i.e., $X_{s}^{*}=Z^{*}(v)$ ) holds, then, by the 2-connectedness assumption, we must have $\sharp(\mathcal{E}) \leq 1$. In this case, one verifies the injectivity assertion directly. Thus, we shall assume that $\mathcal{V} \supsetneq\{v\}$ holds, and take $v^{\prime} \in \mathcal{V}-\{v\}$. Then, replacing $S$ by a suitable finite extension, we may assume that $X^{*} \rightarrow S$ admits a section $\sigma: S \rightarrow X$, such that $\sigma(S)_{\bar{s}} \subset Z^{*}\left(v^{\prime}\right) \cap\left(X_{\bar{s}}^{*}\right)^{\mathrm{sm}}$. Then $\left(X^{*}, \sigma(S)\right)$ is a stable marked curve of type $(g, 1)$ over $S$. Since $\mathcal{G}=\mathcal{G}_{X_{\mathrm{s}}^{*}}$ is 2-connected at $v$, one verifies immediately that $\mathcal{G}_{\left(X_{s}^{*}, \sigma(S)_{\bar{s}}\right)}^{*}$ is also 2-connected at $v$. Thus, by the preceding argument, the natural map

$$
\pi_{1}^{\mathrm{t}}(Z(v))^{\mathrm{ab}} \otimes \mathbb{Z} / N \mathbb{Z} \rightarrow \pi_{1}^{\log }\left(X_{s^{\mathrm{t}}}^{*}, \sigma(S)_{s^{\mathrm{t}}}\right)^{\mathrm{ab}} \otimes \mathbb{Z} / N \mathbb{Z}
$$

is injective.

Now, note that

$$
\pi_{1}^{\log }\left(X_{s^{t}}^{*}, \sigma(S)_{s^{t}}\right)^{\mathrm{ab}} \stackrel{\sim}{\rightarrow} \pi_{1}^{\log }\left(X_{s^{t}}^{*}\right)^{\mathrm{ab}}
$$

holds. (For example, this can be derived from the fact that the natural map $\pi_{1}^{\mathrm{t}}\left(X_{\bar{K}}^{*}, \sigma(S)_{\bar{K}}\right)^{\mathrm{ab}} \rightarrow \pi_{1}\left(X_{\bar{K}}^{*}\right)^{\mathrm{ab}}$ is an isomorphism, via the specialization maps.) 
From these observations, we can finally show the desired injectivity of the natural map

$$
\pi_{1}^{\mathrm{t}}(Z(v))^{\mathrm{ab}} \otimes \mathbb{Z} / N \mathbb{Z} \rightarrow \pi_{1}^{\log }\left(X_{s^{\mathrm{t}}}^{*}\right)^{\mathrm{ab}} \otimes \mathbb{Z} / N \mathbb{Z} .
$$

This completes the proof.

Corollary 3.7. The following conditions are all equivalent.

(i) $\left(X_{s}^{*}, D_{s}\right)^{\log }$ is abelian-injective.

$\left(^{\prime}\right)$ For each $v \in \mathcal{V}$ and each natural number $N$, the natural homomorphism

$$
\pi_{1}^{\mathrm{t}}(Z(v))^{\mathrm{ab}} \otimes \mathbb{Z} / N \mathbb{Z} \rightarrow \pi_{1}^{\log }\left(X_{s^{\mathrm{t}}}^{*}, D_{s^{\mathrm{t}}}\right)^{\mathrm{ab}} \otimes \mathbb{Z} / N \mathbb{Z}
$$

is injective.

(ii) The graph $\mathcal{G}^{*}$ is 2-connected, i.e., $\mathcal{G}^{*}-\{v\}$ is (either empty or) connected for each $v \in \mathcal{V}^{*}$.

Proof. This is a formal consequence of Proposition 3.4, if we replace " $v \in$ $\mathcal{V}^{*}$ " in the statement of (ii) by " $v \in \mathcal{V}$ ". However, since $\mathcal{G}^{*}-\left\{v_{\infty}\right\}$ is always connected, this difference does not affect the validity of the equivalence.

Corollary 3.8. There exists an admissible covering of $\left(X^{*}, D\right)$ (over a finite extension of $S$ ) whose log special fiber is abelian-injective.

Proof. By Remark 3.3, one verifies immediately that there exists an admissible covering $\left(\left(X^{\prime}\right)^{*}, D^{\prime}\right)$ of $\left(X^{*}, D\right)$, such that the dual graph $\mathcal{G}^{\prime} \stackrel{\text { def }}{=}$ $\mathcal{G}_{\left(X^{\prime}\right) \frac{*}{s}, D_{s}^{\prime}}=\left(\mathcal{V}^{\prime}, \mathcal{E}^{\prime}\right)$ has the property that $\nu\left(v^{\prime}\right) \geq 2$ holds for each $v^{\prime} \in \mathcal{V}^{\prime}$.

Next, let $h: \mathcal{G}^{\prime \prime} \rightarrow \mathcal{G}^{\prime}$ be the covering of graphs corresponding to $\pi_{1}\left(\mathcal{G}^{\prime}\right) \rightarrow$ $H \stackrel{\text { def }}{=} \pi_{1}\left(\mathcal{G}^{\prime}\right)^{\mathrm{ab}} \otimes \mathbb{Z} / N \mathbb{Z}$ for some $N \geq 2$. We claim that $\mathcal{G}^{\prime \prime}$ is 2-connected. Indeed, take any vertex $v_{0}^{\prime \prime}$ of $\mathcal{G}^{\prime \prime}$. We shall prove that $\mathcal{G}^{\prime \prime}-\left\{v_{0}^{\prime \prime}\right\}$ is connected. We denote by $v_{0}^{\prime}$ the image of $v_{0}^{\prime \prime}$ in $\mathcal{G}^{\prime}$. We define $\mathcal{C}^{\prime}$ to be the set of connected components of $\mathcal{G}^{\prime}-\left\{v_{0}^{\prime}\right\}$. For each $C^{\prime} \in \mathcal{C}^{\prime}$, let $H_{C^{\prime}}$ denote the image of $\pi_{1}\left(C^{\prime} v_{0}^{\prime}\right)$ in $H\left(C^{\prime} v_{0}^{\prime} \stackrel{\text { def }}{=} C^{\prime} \cup\left\{v_{0}^{\prime}\right\}\right)$, i.e., the decomposition group of $C^{\prime} v_{0}^{\prime}$ in $H$. Since $\pi_{1}\left(\mathcal{G}^{\prime}\right)=\pi_{1}\left(\mathcal{G}^{\prime}, v_{0}^{\prime}\right)$ is isomorphic to the free product of $\pi_{1}\left(C^{\prime} v_{0}^{\prime}\right)=$ $\pi_{1}\left(C^{\prime} v_{0}^{\prime}, v_{0}^{\prime}\right)\left(C^{\prime} \in \mathcal{C}^{\prime}\right)$, we have $H_{C^{\prime}}=\pi_{1}\left(C^{\prime} v_{0}^{\prime}\right)^{\text {ab }} \otimes \mathbb{Z} / N \mathbb{Z}$ for each $C^{\prime} \in \mathcal{C}^{\prime}$, and $H=\prod_{C^{\prime} \in \mathcal{C}^{\prime}} H_{C^{\prime}}$. Moreover, $H_{C^{\prime}}$ is nontrivial for each $C^{\prime} \in \mathcal{C}^{\prime}$. Indeed, otherwise, $C^{\prime} v_{0}^{\prime}$ must be a tree, which implies the existence of a vertex $v^{\prime} \in C^{\prime}$ with $\nu\left(v^{\prime}\right)=1$. This contradicts our choice of $\mathcal{G}^{\prime}$.

For any edge $e^{\prime}$ of $\mathcal{G}^{\prime}$ that meets $v_{0}^{\prime}$, there exists a unique component $C^{\prime}\left(e^{\prime}\right) \in \mathcal{C}^{\prime}$, such that $e^{\prime} \subset C^{\prime}\left(e^{\prime}\right) v_{0}^{\prime}$. Now, by using the property that $\nu\left(v^{\prime}\right) \geq 2$ 
holds for each $v^{\prime} \in \mathcal{V}^{\prime}$, one verifies easily that there exists a loop $l_{e^{\prime}}$ in $C^{\prime}\left(e^{\prime}\right) v_{0}^{\prime}$ that starts and ends with $v_{0}^{\prime}$, such that $e^{\prime}$ is the first edge appearing in $l_{e^{\prime}}$, that the vertex $v_{0}^{\prime}$ appears in $l_{e^{\prime}}$ only as the starting and ending point, and that the image of $l_{e^{\prime}}$ in $H_{C^{\prime}\left(e^{\prime}\right)}=\pi_{1}\left(C^{\prime}\left(e^{\prime}\right) v_{0}^{\prime}\right)^{\mathrm{ab}} \otimes \mathbb{Z} / N \mathbb{Z}$ is nontrivial.

Let $e_{1}^{\prime} \neq e_{2}^{\prime}$ be a pair of distinct edges that meet $v_{0}^{\prime}$, and let $e_{1}^{\prime \prime}$ (resp. $e_{2}^{\prime \prime}$ ) denote the unique edge of $\mathcal{G}^{\prime \prime}$ above $e_{1}^{\prime}$ (resp. $e_{2}^{\prime}$ ) that meets $v_{0}^{\prime \prime}$. If $C^{\prime}\left(e_{1}^{\prime}\right) \neq$ $C^{\prime}\left(e_{2}^{\prime}\right)$ holds, one verifies easily that the unique lifting to $\mathcal{G}^{\prime \prime}$, starting with $v_{0}^{\prime \prime}$, of the path $l_{e_{1}^{\prime}} l_{e_{2}^{\prime}} l_{e_{1}^{\prime}}^{-1} l_{e_{2}^{\prime}}^{-1}$ of $\mathcal{G}^{\prime}$ is a loop $l_{e_{1}^{\prime \prime}, e_{2}^{\prime \prime}}$, such that $e_{1}^{\prime \prime}$ (resp. $e_{2}^{\prime \prime}$ ) is the first (resp. last) edge appearing in $l_{e_{1}^{\prime \prime}, e_{2}^{\prime \prime}}$, and that $v_{0}^{\prime \prime}$ appears in $l_{e_{1}^{\prime \prime}, e_{2}^{\prime \prime}}$ only as the starting and ending point. On the other hand, if $C^{\prime}\left(e_{1}^{\prime}\right)=C^{\prime}\left(e_{2}^{\prime}\right)$ holds, one verifies directly that there exists a loop $l_{e_{1}^{\prime}, e_{2}^{\prime}}$ of $\mathcal{G}^{\prime}$, such that $e_{1}^{\prime}$ (resp. $e_{2}^{\prime}$ ) is the first (resp. last) edge appearing in $l_{e_{1}^{\prime}, e_{2}^{\prime}}$, and that $v_{0}^{\prime}$ appears in $l_{e_{1}^{\prime}, e_{2}^{\prime}}$ only as the starting and ending point. Let $m$ denote the order of the image of $l_{e_{1}^{\prime}, e_{2}^{\prime}}$ in $H$. Then the unique lifting to $\mathcal{G}^{\prime \prime}$, starting with $v_{0}^{\prime \prime}$, of the path $\left(l_{e_{1}^{\prime}, e_{2}^{\prime}}\right)^{m}$ of $\mathcal{G}^{\prime}$ is a loop $l_{e_{1}^{\prime \prime}, e_{2}^{\prime \prime}}$, such that $e_{1}^{\prime \prime}$ (resp. $\left.e_{2}^{\prime \prime}\right)$ is the first (resp. last) edge appearing in $l_{e_{1}^{\prime \prime}, e_{2}^{\prime \prime}}$, and that $v_{0}^{\prime \prime}$ appears in $l_{e_{1}^{\prime \prime}, e_{2}^{\prime \prime}}$ only as the starting and ending point. In both cases, $l_{e_{1}^{\prime \prime}, e_{2}^{\prime \prime}}$ consists of $e_{1}^{\prime \prime}$, a path $p_{e_{1}^{\prime \prime}, e_{2}^{\prime \prime}}$ that does not meet $v_{0}^{\prime \prime}$, and $e_{2}^{\prime \prime}$.

Now, take any pair $v_{1}^{\prime \prime}, v_{2}^{\prime \prime}$ of vertices of $\mathcal{G}^{\prime \prime}-\left\{v_{0}^{\prime \prime}\right\}$. We shall prove that there exists a path in $\mathcal{G}^{\prime \prime}-\left\{v_{0}^{\prime \prime}\right\}$ that connects $v_{1}^{\prime \prime}$ and $v_{2}^{\prime \prime}$. Since $\mathcal{G}^{\prime \prime}$ is connected, there exists a path $p$ in $\mathcal{G}^{\prime \prime}$ that connects $v_{1}^{\prime \prime}$ and $v_{2}^{\prime \prime}$. By induction on the number of times that $p$ meets $v_{0}^{\prime \prime}$, we may reduce the problem to the case where $p$ meets $v_{0}^{\prime \prime}$ only once. Then $p$ contains a unique successive pair of edges $e_{1}^{\prime \prime}, e_{2}^{\prime \prime}$ that meet $v_{0}^{\prime \prime}$. First, assume $e_{1}^{\prime \prime}=e_{2}^{\prime \prime}$. Then, just removing this pair from $p$, we obtain a new path from $v_{1}^{\prime \prime}$ to $v_{2}^{\prime \prime}$ that does not meet $v_{0}^{\prime \prime}$. Next, assume $e_{1}^{\prime \prime} \neq e_{2}^{\prime \prime}$. In

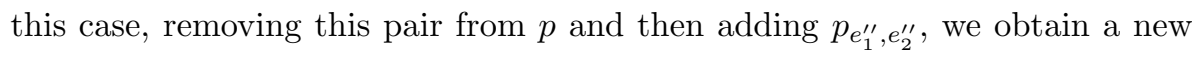
path from $v_{1}^{\prime \prime}$ to $v_{2}^{\prime \prime}$ that does not meet $v_{0}^{\prime \prime}$. This completes the proof of the 2-connectedness of $\mathcal{G}^{\prime \prime}$.

Now, let $\left(\left(X^{\prime \prime}\right)^{*}, D^{\prime \prime}\right)$ be the (combinatorial) étale covering of $\left(\left(X^{\prime}\right)^{*}, D^{\prime}\right)$ corresponding to $\mathcal{G}^{\prime \prime} \rightarrow \mathcal{G}^{\prime}$. By definition, the dual graph $\mathcal{G}_{\left(X^{\prime \prime}\right) \frac{*}{s}}$ coincides with $\mathcal{G}^{\prime \prime}$. Thus, $\mathcal{G}_{\left(X^{\prime \prime}\right) \frac{*}{s}}$ is 2-connected, hence so is $\mathcal{G}_{\left(\left(X^{\prime \prime}\right) \frac{*}{s}, D \frac{\prime \prime}{s}\right)}^{*}$, as desired.

Observe that we have

$$
\gamma_{l}\left(\pi_{1}^{\log }\left(X_{s^{\mathrm{t}}}, D_{s^{\mathrm{t}}}\right)\right)= \begin{cases}2 g+r-1+b^{(2)}=\gamma_{l}\left(X_{K}^{*}\right)+r-1+b^{(2)}, & l \neq p, \\ \gamma_{p}\left(X_{s}^{*}\right), & l=p(>0) .\end{cases}
$$

Now, for each natural number $N$, we set

$$
\gamma_{l}^{\mathrm{av}}(N)\left(\left(X_{s}^{*}, D_{s}\right)^{\log }\right) \stackrel{\text { def }}{=} \gamma_{l}^{\mathrm{av}}(N)\left(\pi_{1}^{\log }\left(X_{s^{\mathrm{t}}}^{*}, D_{s^{\mathrm{t}}}\right)\right) .
$$


Then we have:

Theorem 3.10. Assume $p>0$, and that $\left(X_{s}^{*}, D_{s}\right)^{\log }$ is abelianinjective. Then we have

$$
\lim _{f \rightarrow \infty} \gamma_{p}^{\mathrm{av}}\left(p^{f}-1\right)\left(\left(X_{s}^{*}, D_{s}\right)^{\log }\right)=g-h_{1}(\mathcal{G})-\sharp\left(\mathcal{V}_{\nu^{*} \leq 1}\right)
$$

Here, $h_{1}(\mathcal{G}) \stackrel{\text { def }}{=} \operatorname{rk}_{\mathbb{Z}}\left(H_{1}(\mathcal{G}, \mathbb{Z})\right)$ and $\mathcal{V}_{\nu * \leq 1} \stackrel{\text { def }}{=} \sharp\left\{v \in \mathcal{V} \mid \nu^{*}(v) \leq 1\right\}$.

Proof. Let $N$ denote a natural number that is not divisible by $p$. By (3.9), (2.7), Remark 2.8, and Proposition 3.4, we deduce

$$
\begin{aligned}
& \gamma_{p}\left(\pi_{1}^{\log }\left(X_{s^{\mathrm{t}}}^{*}, D_{s^{\mathrm{t}}}\right)(N)\right) \\
& =\sum_{v \in \mathcal{V}}\left(\pi_{1}^{\log }\left(X_{s^{\mathrm{t}}}^{*}, D_{s^{\mathrm{t}}}\right)^{\mathrm{ab}} \otimes \mathbb{Z} / N \mathbb{Z}: \pi_{1}^{\mathrm{t}}(Z(v))^{\mathrm{ab}} \otimes \mathbb{Z} / N \mathbb{Z}\right) \gamma_{p}\left(\pi_{1}^{\mathrm{t}}(Z(v))(N)\right) \\
& \quad+h_{1}(\mathcal{G}(N)),
\end{aligned}
$$

where $\mathcal{G}(N)$ denotes the covering of $\mathcal{G}$ corresponding to the quotient $H_{1}(\mathcal{G}, \mathbb{Z}) \otimes$ $\mathbb{Z} / N \mathbb{Z}$ of $\pi_{1}(\mathcal{G})$. Dividing by $\sharp\left(\pi_{1}^{\log }\left(X_{s^{t}}^{*}, D_{s^{t}}\right)^{\text {ab }} \otimes \mathbb{Z} / N \mathbb{Z}\right)=N^{2 g+r-1+b^{(2)}}$, we have

$$
\gamma_{p}^{\mathrm{av}}(N)\left(\left(X_{s}^{*}, D_{s}\right)^{\log }\right)=\sum_{v \in \mathcal{V}} \gamma_{p}^{\mathrm{av}}(N)(Z(v))+\frac{1}{N^{2 g+r-1+b^{(2)}}} h_{1}(\mathcal{G}(N))
$$

For each $v \in \mathcal{V}$, we denote by $g_{\left(Z^{*}\right)} \sim$ the genus of the proper, smooth curve $\left(Z^{*}\right)^{\sim}$ over $\bar{s}$. By definition, the cardinality of the point set $\left(Z^{*}\right)^{\sim}-Z$ is $\nu^{*}\left(Z^{*}\right)$. Thus, we set

$$
g_{\left(Z^{*}\right) \sim}^{\prime} \stackrel{\text { def }}{=} \begin{cases}g_{\left(Z^{*}\right)}-1, & \nu^{*}\left(Z^{*}\right) \leq 1 \\ g_{\left(Z^{*}\right)}, & \nu^{*}\left(Z^{*}\right)>1\end{cases}
$$

On the other hand, by (a graph-theoretic version of) Schreier's theorem, we have

$$
h_{1}(\mathcal{G}(N))=N^{h_{1}(\mathcal{G})}\left\{h_{1}(\mathcal{G})-1\right\}+1 .
$$


Now, observing that $2 g+r-1+b^{(2)}>g \geq h_{1}(\mathcal{G})$ holds, we obtain

$$
\begin{aligned}
& \lim _{f \rightarrow \infty} \gamma_{p}^{\mathrm{av}}\left(p^{f}-1\right)\left(\left(X_{s}^{*}, D_{s}\right)^{\log }\right) \\
= & \sum_{v \in \mathcal{V}} \lim _{f \rightarrow \infty} \gamma_{p}^{\mathrm{av}}\left(p^{f}-1\right)(Z(v)) \\
= & \sum_{v \in \mathcal{V}} g_{\left(Z^{*}\right) \sim(v)}^{\prime} \\
= & \left(\sum_{v \in \mathcal{V}} g_{\left(Z^{*}\right) \sim(v)}\right)-\sharp\left(\mathcal{V}_{\nu^{*} \leq 1}\right) \\
= & g-h_{1}(\mathcal{G})-\sharp\left(\mathcal{V}_{\nu^{*} \leq 1}\right) .
\end{aligned}
$$

Here, the second equality follows from Theorem 3.1.

Let the notations and the assumptions be as in Theorem 3.10, and assume, moreover, that the characteristic of $K$ is $p$. For each natural number $N$, let $\left(X_{\bar{K}}^{*}(N), D_{\bar{K}}(N)\right)$ be the (tame) covering of $\left(X_{\bar{K}}^{*}, D_{\bar{K}}\right)$ corresponding to the open subgroup $\pi_{1}^{\mathrm{t}}\left(X_{\bar{K}}^{*}, D_{\bar{K}}\right)(N)$ of $\pi_{1}^{\mathrm{t}}\left(X_{\bar{K}}^{*}, D_{\bar{K}}\right)$. This covering admits a stable model $\left(X^{*}(N), D(N)\right)$ over a suitable (finite) extension of $S$. We set

$$
\delta_{p}^{\mathrm{av}}(N)\left(X^{*}, D\right) \stackrel{\text { def }}{=} \frac{\delta_{p}\left(X^{*}(N)\right)}{\sharp\left(\pi_{1}^{\log }\left(X_{s^{\mathrm{t}}}^{*}, D_{s^{\mathrm{t}}}\right)^{\mathrm{ab}} \otimes \mathbb{Z} / N \mathbb{Z}\right)} .
$$

Then we have:

\section{Corollary 3.11.}

$$
\lim _{f \rightarrow \infty} \delta_{p}^{\mathrm{av}}\left(p^{f}-1\right)\left(X^{*}, D\right)= \begin{cases}h_{1}(\mathcal{G})+\sharp\left(\mathcal{V}_{\nu^{*} \leq 1}\right)-1, & r \leq 1, \\ h_{1}(\mathcal{G})+\sharp\left(\mathcal{V}_{\nu^{*} \leq 1}\right), & r>1 .\end{cases}
$$

Proof. Apply Theorem 3.1 and Theorem 3.10 to the generic fiber and the log special fiber, respectively, and take the difference.

Remark 3.12. Even without the assumption that $\left(X_{s}^{*}, D_{s}\right)^{\log }$ is abelian-injective, one can obtain an upper bound (resp. a lower bound) of $\varlimsup_{f \rightarrow \infty} \gamma_{p}^{\text {av }}\left(p^{f}-1\right)\left(\left(X_{s}^{*}, D_{s}\right)^{\log }\right)\left(\right.$ resp. $\left.\varliminf_{f \rightarrow \infty} \delta_{p}^{\text {av }}\left(p^{f}-1\right)\left(X^{*}, D\right)\right)$. To do this, one needs not only the statement of Theorem 3.1 but also some arguments in the proof of Theorem 3.1 in [Tam2]. 
$\langle$ Specialization theorems on fundamental groups of curves $\rangle$

Let $R$ be a complete discrete valuation ring, and set $S \stackrel{\text { def }}{=} \operatorname{Spec}(R)$. Let $K$ and $k$ denote the field of fractions and the residue field, respectively, of $R$, and set $\eta \stackrel{\text { def }}{=} \operatorname{Spec}(K)$ and $s \stackrel{\text { def }}{=} \operatorname{Spec}(k)$. Let $\left(X^{*}, D\right)$ be a smooth, stable marked curve over $S$. Then we have the specialization homomorphism

$$
\operatorname{sp}^{\mathrm{t}}: \pi_{1}^{\mathrm{t}}\left(X_{\bar{\eta}}, D_{\bar{\eta}}\right) \rightarrow \pi_{1}^{\mathrm{t}}\left(X_{\bar{s}}, D_{\bar{s}}\right) .
$$

Theorem 3.13 ([Tam3], Theorem (0.3)). Assume $p \stackrel{\text { def }}{=} \operatorname{char}(K)=$ $\operatorname{char}(k)>0$, and that $\left(X_{\bar{\eta}}, D_{\bar{\eta}}\right)$ is not (resp. $\left(X_{\bar{s}}, D_{\bar{s}}\right)$ is) $\mathbb{F}_{p}$-isotrivial. Then $\mathrm{sp}^{\mathrm{t}}$ is not an isomorphism.

In the case where we do not assume that the special fiber is $\mathbb{F}_{p}$-isotrivial, we have the following partial result due to Saïdi. Here, let $k$ be a field of characteristic $p>0$, and $S$ a proper, smooth, geometrically connected curve over $k$. Let $\eta$ denote the generic point of $S$. Let $\left(X^{*}, D\right)$ be a (an everywhere) smooth, stable marked curve over $S$. Then we have:

Theorem 3.14 ([Sa]). Assume that $\left(X^{*}, D\right)$ is not $k$-isotrivial. Then there exists a closed point $s \in S$, such that the specialization homomorphism

$$
\operatorname{sp}^{\mathrm{t}}: \pi_{1}^{\mathrm{t}}\left(X_{\bar{\eta}}, D_{\bar{\eta}}\right) \rightarrow \pi_{1}^{\mathrm{t}}\left(X_{\bar{s}}, D_{\bar{s}}\right)
$$

is not an isomorphism.

Remark 3.15. The author thinks that it is natural and desirable to be able to remove the assumption in Theorem 3.14 that $S$ is proper over $k$.

〈The Tate-de Jong theorem on p-divisible groups and its application〉

Theorem 3.16 ([Tat], [D2]). Let $S$ be a noetherian, normal, integral scheme, $K$ the function field of $S$, and $\eta \stackrel{\text { def }}{=} \operatorname{Spec}(K)$. Let $p$ be a prime number. Let $G$ and $H$ be p-divisible groups over $S$. Then the natural map

$$
\operatorname{Hom}_{S}(G, H) \rightarrow \operatorname{Hom}_{\eta}\left(G_{\eta}, H_{\eta}\right)
$$

is an isomorphism.

Remark 3.17. If $p$ is invertible on $S$, Theorem 3.16 is immediate. In general, Theorem 3.16 was proved in [Tat] (resp. [D2]) for $p \neq \operatorname{char}(K)$ (resp. $p=\operatorname{char}(K))$. 
We shall apply this result to Galois representations associated with abelian varieties. Let $R$ be a complete discrete valuation ring, and let $K$ and $k$ denote the field of fractions and the residue field, respectively, of $R$. We assume that $k$ is separably closed (or, equivalently, $R$ is strictly henselian), and set $I \stackrel{\text { def }}{=} G_{K}$. Let $A$ be a generically proper, semi-abelian scheme over $R$. Let $l$ be a prime number, and we set $V_{l}(A(\bar{K})) \stackrel{\text { def }}{=} T_{l}(A(\bar{K})) \otimes_{\mathbb{Z}_{l}} \mathbb{Q}_{l}$, which is a $\mathbb{Q}_{l}$-vector space of dimension $\gamma_{l}\left(A_{K}\right)$ that admits a natural action by $I$. For a $\mathbb{Q}_{l}[I]$-module $V$, we define the coinvariant space $V_{I}$ to be the maximal quotient $\mathbb{Q}_{l}$-vector space of $V$ on which $I$ acts trivially.

Theorem 3.18.

$$
\operatorname{dim}_{\mathbb{Q}_{l}}\left(V_{l}(A(\bar{K}))_{I}\right)=\gamma_{l}\left(A_{k}\right)
$$

Proof. We have

$$
\begin{aligned}
& \operatorname{Hom}_{\mathbb{Q}_{l}}\left(V_{l}(A(\bar{K}))_{I}, \mathbb{Q}_{l}\right) \\
= & \operatorname{Hom}_{\mathbb{Q}_{l}[I]}\left(V_{l}(A(\bar{K})), \mathbb{Q}_{l}\right) \\
= & \operatorname{Hom}_{\mathbb{Z}_{l}[I]}\left(T_{l}(A(\bar{K})), \mathbb{Z}_{l}\right) \otimes_{\mathbb{Z}_{l}} \mathbb{Q}_{l} \\
= & \operatorname{Hom}_{\mathbb{Z}_{l}[I]}\left(A\left[l^{\infty}\right]_{K}(\bar{K}), \mathbb{Q}_{l} / \mathbb{Z}_{l}\right) \otimes_{\mathbb{Z}_{l}} \mathbb{Q}_{l} \\
= & \operatorname{Hom}_{\mathbb{Z}_{l}[I]}\left(\left(A\left[l^{\infty}\right]_{K}\right)^{\text {ét }}\left(K^{\mathrm{sep}}\right), \mathbb{Q}_{l} / \mathbb{Z}_{l}\right) \otimes_{\mathbb{Z}_{l}} \mathbb{Q}_{l} \\
= & \operatorname{Hom}_{K}\left(\left(A\left[l^{\infty}\right]_{K}\right)^{\text {ét }}, \mathbb{Q}_{l} / \mathbb{Z}_{l}\right) \otimes_{\mathbb{Z}_{l}} \mathbb{Q}_{l} \\
= & \operatorname{Hom}_{K}\left(A\left[l^{\infty}\right]_{K}, \mathbb{Q}_{l} / \mathbb{Z}_{l}\right) \otimes_{\mathbb{Z}_{l}} \mathbb{Q}_{l} \\
= & \operatorname{Hom}_{K}\left(\mathbb{G}_{m}\left[l^{\infty}\right]_{K}, A^{\vee}\left[l^{\infty}\right]_{K}\right) \otimes_{\mathbb{Z}_{l}} \mathbb{Q}_{l} \\
= & \operatorname{Hom}_{K}\left(\mathbb{G}_{m}\left[l^{\infty}\right]_{K},\left(A^{\vee}\left[l^{\infty}\right]^{\mathrm{f}}\right)_{K}\right) \otimes_{\mathbb{Z}_{l}} \mathbb{Q}_{l} \\
= & \operatorname{Hom}_{R}\left(\mathbb{G}_{m}\left[l^{\infty}\right]_{R}, A^{\vee}\left[l^{\infty}\right]^{\mathrm{f}}\right) \otimes_{\mathbb{Z}_{l}} \mathbb{Q}_{l} \\
= & \operatorname{Hom}_{R}\left(\left(A^{\vee}\left[l^{\infty}\right]^{\mathrm{f}}\right)^{\vee}, \mathbb{Q}_{l} / \mathbb{Z}_{l}\right) \otimes_{\mathbb{Z}_{l}} \mathbb{Q}_{l} \\
= & \operatorname{Hom}_{R}\left(\left(\left(A^{\vee}\left[l^{\infty}\right]^{\mathrm{f}}\right)^{\vee}\right)^{\mathrm{e} t}, \mathbb{Q}_{l} / \mathbb{Z}_{l}\right) \otimes_{\mathbb{Z}_{l}} \mathbb{Q}_{l} \\
= & \operatorname{Hom}_{k}\left(\left(\left(\left(A^{\vee}\left[l^{\infty}\right]^{\mathrm{f}}\right)^{\vee}\right)^{\mathrm{ét}}\right)_{k}, \mathbb{Q}_{l} / \mathbb{Z}_{l}\right) \otimes_{\mathbb{Z}_{l}} \mathbb{Q}_{l} \\
= & \operatorname{Hom}_{k}\left(\left(\left(\left(A^{\vee}\left[l^{\infty}\right]^{\mathrm{f}}\right)^{\vee}\right)_{k}\right)^{\mathrm{ét}}, \mathbb{Q}_{l} / \mathbb{Z}_{l}\right) \otimes_{\mathbb{Z}_{l}} \mathbb{Q}_{l} \\
= & \operatorname{Hom}_{k}\left(\left(\left(A^{\vee}\left[l^{\infty}\right]^{\mathrm{f}}\right)^{\vee}\right)_{k}, \mathbb{Q}_{l} / \mathbb{Z}_{l}\right) \otimes_{\mathbb{Z}_{l}} \mathbb{Q}_{l} \\
= & \operatorname{Hom}_{k}\left(\left(\left(A^{\vee}\left[l^{\infty}\right]^{\mathrm{f}}\right)_{k}\right)^{\vee}, \mathbb{Q}_{l} / \mathbb{Z}_{l}\right) \otimes_{\mathbb{Z}_{l}} \mathbb{Q}_{l} \\
= & \operatorname{Hom}_{k}\left(\mathbb{G}_{m}\left[l^{\infty}\right]_{k},\left(A^{\vee}\left[l^{\infty}\right]^{\mathrm{f}}\right)_{k}\right) \otimes_{\mathbb{Z}_{l}} \mathbb{Q}_{l} \\
= & \operatorname{Hom}_{k}\left(\mathbb{G}_{m}\left[l^{\infty}\right]_{k},\left(A_{k}^{\vee}\right)\left[l^{\infty}\right]\right) \otimes_{\mathbb{Z}_{l}} \mathbb{Q}_{l} .
\end{aligned}
$$

Here, $A\left[l^{\infty}\right]^{\mathrm{f}}$ denotes the fixed part of $A\left[l^{\infty}\right]$ in the sense of [SGA7], Exposé IX. (Namely, $A\left[l^{\infty}\right]^{\mathrm{f}}$ is the maximal divisible subgroup of $A\left[l^{\infty}\right]$ that is "indfinite" over $R$, i.e., each layer is finite over $R$.) $A_{K}^{\vee}$ denotes the dual abelian 
variety of $A_{K}$, and $A^{\vee}$ denotes the group subscheme of the Néron model of $A_{K}^{\vee}$ obtained by removing all connected components of the special fiber but the one containing the origin. For an $l$-divisible group $G, G^{\vee}$ denotes the Cartier dual of $G$. For an $l$-divisible group $G=\left(G_{n}\right)_{n \geq 0}$ over a complete noetherian local ring, $G^{\text {ét }}$ denotes $G / G^{0}$, where $G^{0}$ is the connected component of $G$ containing the origin. (More precisely, $G^{0} \stackrel{\text { def }}{=}\left(\left(G_{n}\right)^{0}\right)_{n \geq 0}$, where $\left(G_{n}\right)^{0}$ is the connected component of $G_{n}$ containing the origin.) Note that the ninth equality follows from Theorem 3.16 .

Thus, we obtain

$$
\operatorname{dim}_{\mathbb{Q}_{l}}\left(V_{l}(A(\bar{K}))_{I}\right)=\gamma_{l}\left(A_{k}^{\vee}\right)=\gamma_{l}\left(A_{k}\right),
$$

as desired, where the second equality follows from the fact that $A_{k}^{\vee}$ and $A_{k}$ are isogenous to each other over $k$.

Corollary 3.19. If $\delta_{l}(A)>0$, then the image of the Galois representation $\rho: I \rightarrow \operatorname{Aut}\left(T_{l}(A(\bar{K}))\right)$ is infinite.

Proof. Suppose otherwise, then, replacing $K$ by a suitable finite extension, we may assume that $\rho$ is trivial. Then we have $V_{l}(A(\bar{K}))_{I}=V_{l}(A(\bar{K}))$, hence $\gamma_{l}\left(A_{k}\right)=\gamma_{l}\left(A_{K}\right)$ by Theorem 3.18, i.e., $\delta_{l}(A)=0$.

\section{$\S 4$. Singularities of Coverings of Stable Curves}

In this $\S$, we investigate singularities of coverings of stable curves.

Let $S$ be a 1-dimensional, noetherian, normal, integral, separated scheme, and denote by $K$ the function field of $S$. Let $\left(X^{*}, D\right)$ be a stable marked curve over $S$, and assume that $\left(X^{*}, D\right)$ is generically smooth.

Proposition 4.1. Let $s$ be a closed point of $S$ and let $p \geq 0$ be the characteristic of the residue field of $s$. Consider the following conditions:

(Sing) There exist a finite extension $\left(S^{\prime}, K^{\prime}\right)$ of $(S, K)$, a generically tame, stable covering $\left(Y_{S^{\prime}}^{*}, E_{S^{\prime}}\right)$ of $\left(X_{S^{\prime}}^{*}, D_{S^{\prime}}\right)$ over $S^{\prime}$, such that the image of $\left(Y_{S^{\prime}}^{*}\right)^{\text {non-sm }}$ in $S$ contains $s$.

(Jac1) There exist a finite extension $\left(S^{\prime}, K^{\prime}\right)$ of $(S, K)$ and a generically tame, stable covering $\left(Y_{S^{\prime}}^{*}, E_{S^{\prime}}\right)$ of $\left(X_{S^{\prime}}^{*}, D_{S^{\prime}}\right)$ over $S^{\prime}$, such that the Jacobian variety $J_{Y_{K^{\prime}}^{*}}$ of $Y_{K^{\prime}}^{*}$ has bad reduction at some $s^{\prime} \in S^{\prime}$ above $s$.

(Jac2) There exist a finite extension $\left(S^{\prime}, K^{\prime}\right)$ of $(S, K)$ and a generically tame, stable covering $\left(Y_{S^{\prime}}^{*}, E_{S^{\prime}}\right)$ of $\left(X_{S^{\prime}}^{*}, D_{S^{\prime}}\right)$ over $S^{\prime}$, such that $Y_{S^{\prime}}^{*}$ has positive $p$ defect at some $s^{\prime} \in S^{\prime}$ above $s$ in the sense that $(p>0$ and $) \delta_{p}\left(Y_{S^{\prime}}^{*}\right.$ $\left.\times_{S^{\prime}} \operatorname{Spec}\left(\mathcal{O}_{S^{\prime}, s^{\prime}}\right)\right)>0$ for some $s^{\prime} \in S^{\prime}$ above $s$. 
(Jac3) There exist a finite extension $\left(S^{\prime}, K^{\prime}\right)$ of $(S, K)$ and a generically tame, stable covering $\left(Y_{S^{\prime}}^{*}, E_{S^{\prime}}\right)$ of $\left(X_{S^{\prime}}^{*}, D_{S^{\prime}}\right)$ over $S^{\prime}$, such that, at some $s^{\prime} \in S^{\prime}$ above $s, J_{Y_{K^{\prime}}^{*}}$ has bad reduction and $Y_{S^{\prime}}^{*}$ has positive $p$-defect.

(Sp1) The specialization map $\pi_{1}^{\mathrm{t}}\left(X_{\bar{K}}^{*}, D_{\bar{K}}\right) \rightarrow \pi_{1}^{\mathrm{t}}\left(X_{\bar{s}}^{*}, D_{\bar{s}}\right)$ is not an isomorphism.

(Sp2) The specialization map $\pi_{1}^{\mathrm{t}}\left(X_{\bar{K}}^{*}, D_{\bar{K}}\right) \rightarrow \pi_{1}^{\log }\left(X_{s^{t}}^{*}, D_{s^{t}}\right)$ is not an isomorphism.

(Mon1) The outer Galois representation $\rho: \operatorname{Gal}\left(K^{\mathrm{sep}} / K\right) \rightarrow \operatorname{Out}\left(\pi_{1}^{\mathrm{t}}\left(X_{\bar{K}}^{*}, D_{\bar{K}}\right)\right)$ is ramified at $s$, i.e., the restriction of $\rho$ on the inertia subgroup $I$ at $s$ (defined up to conjugacy) is nontrivial.

(Mon2) $\operatorname{Ker}\left(\left.\rho\right|_{I}\right)$ is contained in the wild inertia group $I^{\mathrm{w}}$.

(Mon3) $\rho\left(I^{\mathrm{w}}\right)$ is infinite.

(Mon4) $\operatorname{Ker}\left(\left.\rho\right|_{I}\right)$ is contained in the wild inertia group $I^{\mathrm{w}}$ and $\rho\left(I^{\mathrm{w}}\right)$ is infinite.

(RamS) (resp. (WRamS)) There exist a finite extension $\left(S^{\prime}, K^{\prime}\right)$ of $(S, K)$, a generically tame, stable coverings $\left(Y_{S^{\prime}}^{*}, E_{S^{\prime}}\right) \rightarrow\left(\left(X^{\prime}\right)_{S^{\prime}}^{*},\left(D^{\prime}\right)_{S^{\prime}}\right) \rightarrow\left(X_{S^{\prime}}^{*}, D_{S^{\prime}}\right)$ over $S^{\prime}$, a point $s^{\prime} \in S^{\prime}$ above $s$, an irreducible component $\left(Z^{\prime}\right)^{*}=\left\{z^{\prime}\right\}^{\text {cl }}$ (see $\S 0$ for the notation "cl") of $\left(X^{\prime}\right) \frac{*}{s^{\prime}}$, and a point $w$ of the fiber $Y_{S^{\prime}}^{*} \underset{\left(X^{\prime}\right)^{*}}{\times} z^{\prime}$, such that the natural morphism from the integral closure $W^{\mathrm{s}}$ of $Z^{\prime}$ in $\boldsymbol{k}(w)^{\mathrm{s}}$ to the normalization $\left(\left(Z^{\prime}\right)^{*}\right)^{\sim}\left(\right.$ in $\left.\boldsymbol{k}\left(z^{\prime}\right)\right)$ of $\left(Z^{\prime}\right)^{*}$ is ramified (resp. wildly ramified) at some point outside $\left(D^{\prime}\right)_{\overline{s^{\prime}}}$ (resp. at some point). Here, $\boldsymbol{k}(w)^{\mathrm{s}} / \boldsymbol{k}\left(z^{\prime}\right)$ denotes the maximal separable subextension of $\boldsymbol{k}(w) / \boldsymbol{k}\left(z^{\prime}\right)$.

(RamG) There exist a finite extension $\left(S^{\prime}, K^{\prime}\right)$ of $(S, K)$, a generically tame, stable covering $\left(Y_{S^{\prime}}^{*}, E_{S^{\prime}}\right)$ of $\left(X_{S^{\prime}}^{*}, D_{S^{\prime}}\right)$ over $S^{\prime}$, an irreducible component $Z^{*}=$ $\{z\}^{\mathrm{cl}}$ of $X_{\bar{s}}^{*}$, and a point $w$ of the fiber $Y_{S^{\prime}}^{*} \underset{X^{*}}{\times} z$, such that $\boldsymbol{k}(w) / \boldsymbol{k}(z)$ is inseparable.

Then:

(i) (Jac1), (Sp1), (Mon1), (Mon2), and (RamS) are equivalent to (Sing).

(ii) If, moreover, $p>0$, all the above conditions but (WRamS) are equivalent to (Sing), and we have the implication (WRamS) $\Longrightarrow$ (Sing).

Remark 4.2. "Sing", "Jac", "Sp", "Mon", "RamS", "WRamS", and "RamG" stand for "singularity", "Jacobian", "specialization", "monodromy", "ramification at a special point", "wild ramification at a special point", and "ramification at a generic point", respectively. 
Proof. (i) (Sing) $\Longrightarrow$ (Jac1). For this, see the last step of the proof of [Tam1], Theorem (5.3). (See also the last step of the proof of [Tam3], Theorem (8.8).)

$(\mathrm{Jac} 1) \Longrightarrow($ Mon2). For this, see the proof of [Tam3], Theorem (8.9).

(Mon2) $\Longrightarrow($ Mon1). Clear.

(Mon1) $\Longrightarrow(\mathrm{Sp} 1)$. Suppose that (Sp1) does not hold, i.e., that the specialization map $\pi_{1}^{\mathrm{t}}\left(X_{\bar{K}}^{*}, D_{\bar{K}}\right) \rightarrow \pi_{1}^{\mathrm{t}}\left(X_{\bar{s}}^{*}, D_{\bar{s}}\right)$ is an isomorphism. Then the outer action of $I$ on $\pi_{1}^{\mathrm{t}}\left(X_{\bar{K}}^{*}, D_{\bar{K}}\right)$ is isomorphic to that on $\pi_{1}^{\mathrm{t}}\left(X_{\bar{s}}^{*}, D_{\bar{s}}\right)$, which is clearly trivial.

(Sp1) $\Longrightarrow$ (Sing). Suppose that (Sing) does not hold. Then, by [Tam1], Lemma (5.5), (ii) $\Longrightarrow$ (i), one verifies immediately that the specialization map $\pi_{1}^{\mathrm{t}}\left(X_{\bar{K}}^{*}, D_{\bar{K}}\right) \rightarrow \pi_{1}^{\mathrm{t}}\left(X_{\bar{s}}^{*}, D_{\bar{s}}\right)$ is an isomorphism.

(Sing) $\Longrightarrow($ RamS). By Remark 3.3, given any singular point (which is necessarily outside the cusps), there exists a covering that is (tamely) ramified at that singular point.

(RamS) $\Longrightarrow$ (Sing). Suppose that (Sing) does not hold. Then, by [Tam1], Lemma (5.5), (ii) $\Longrightarrow(\mathrm{i})$, one verifies immediately that (RamS) does not hold.

(ii) (Sing) $\Longrightarrow($ Jac3). Assume that (Sing) holds. Then, by (i), (Jac1) holds. Thus, replacing $\left(X^{*}, D\right)$ by a suitable covering, we may assume that $J_{X_{K}^{*}}$ has bad reduction at $s$. In particular, we have $g=\operatorname{dim}\left(J_{X_{K}^{*}}\right)>0$. First, assume $\operatorname{char}(K)=0$. Then, by $(2.5)$, we have $\delta_{p}\left(X^{*} \times_{S} \operatorname{Spec}\left(\mathcal{O}_{S, s}\right)\right) \geq g>0$, as desired. Next, assume $\operatorname{char}(K)=p$. By a technique similar to the last step of the proof of [Tam1], Theorem $(5.3)$, (replacing $\left(X^{*}, D\right)$ by a suitable tame covering) we may assume that the dual graph $\mathcal{G} \stackrel{\text { def }}{=} \mathcal{G}_{X_{\mathrm{s}}^{*}}$ satisfies $h_{1}(\mathcal{G}) \geq 2$. Note that, once this condition is satisfied, it is also satisfied by all the coverings. Moreover, by Corollary 3.8, again replacing $\left(X^{*}, D\right)$ by a suitable admissible covering, we may also assume that $\left(X_{\bar{s}}^{*}, D_{\bar{s}}\right)^{\log }$ is abelian-injective. Now, Corollary 3.11 implies that there exists an admissible covering $\left(Y^{*}, E\right)$ of $\left(X^{*}, D\right)$ with positive $p$-defect, as desired. (More precisely, we can take $\left(Y^{*}, E\right)=\left(X^{*}\left(p^{f}-1\right), D\left(p^{f}-1\right)\right)$ for sufficiently large f.)

$(\mathrm{Jac} 3) \Longrightarrow(\mathrm{Jac} 2)$. Clear.

$(\mathrm{Jac} 2) \Longrightarrow($ Mon3). In light of Corollary 3.19, this is similar to the proof of (Jac1) $\Longrightarrow$ (Mon2) (cf. [Tam3], Theorem (8.9)). More precisely, assume that (Jac2) holds. Let $I^{\prime}$ denote the inertia subgroup at $s^{\prime}$, which is an open subgroup of $I$. By Corollary 3.19, $I^{\prime}$ has infinite image $P$ in $\operatorname{Aut}\left(\pi_{1}\left(Y_{K}^{*}\right)^{p, \text { ab }}\right)$. Since the latter group is almost pro- $p$ (in the sense that it admits an open pro- $p$ subgroup), we may also assume that $P$ is a pro- $p$ group, replacing $I^{\prime}$ by a suitable 
open subgroup if necessary. On the other hand, by Remark 2.3(ii), $I^{\prime} \cap \operatorname{Ker}\left(\left.\rho\right|_{I}\right)$ has finite image in $\operatorname{Out}\left(\pi_{1}^{\mathrm{t}}\left(Y_{\bar{K}}^{*}, E_{\bar{K}}\right)\right)$, hence finite image $Q$ in $\operatorname{Aut}\left(\pi_{1}\left(Y_{\bar{K}}^{*}\right)^{p, \mathrm{ab}}\right)$, a fortiori. From these observations, it follows that $\rho\left(I^{\prime}\right)=I^{\prime} /\left(I^{\prime} \cap \operatorname{Ker}\left(\left.\rho\right|_{I}\right)\right)$ is surjectively mapped onto $P / Q$, which is an infinite pro- $p$ group. This implies (Mon3).

$(\mathrm{Jac} 3) \Longrightarrow($ Mon4 $)$. This follows from the combination of $(\mathrm{Jac} 3) \Longrightarrow$ $(\mathrm{Jac} 1) \Longrightarrow($ Mon2) and $(\mathrm{Jac} 3) \Longrightarrow(\mathrm{Jac} 2) \Longrightarrow($ Mon3 $)$.

(Mon4) $\Longrightarrow($ Mon3). Clear.

(Mon3) $\Longrightarrow(\mathrm{Sp} 2)$. Suppose that (Sp2) does not hold, i.e., that the specialization map $\pi_{1}^{\mathrm{t}}\left(X_{\bar{K}}^{*}, D_{\bar{K}}\right) \rightarrow \pi_{1}^{\log }\left(X_{s^{\mathrm{t}}}^{*}, D_{s^{\mathrm{t}}}\right)$ is an isomorphism. Then the outer action of $I$ on $\pi_{1}^{\mathrm{t}}\left(X_{\bar{K}}^{*}, D_{\bar{K}}\right)$ is isomorphic to that on $\pi_{1}^{\log }\left(X_{s^{t}}^{*}, D_{s^{t}}\right)$, which factors through the quotient $\pi_{1}^{\log }(s)=I / I^{\mathrm{w}}$, i.e., $\rho\left(I^{\mathrm{w}}\right)=\{1\}$.

$(\mathrm{Sp} 2) \Longrightarrow($ RamG). Suppose that (RamG) does not hold. Then this (together with the purity of the branched locus and Lemma 2.1(i)(ii)(iii)) implies that every tame covering $\left(Y_{K^{\prime}}^{*}, E_{K^{\prime}}^{*}\right)$ of $\left(X_{K^{\prime}}^{*}, D_{K^{\prime}}\right)$ over a finite extension $\left(S^{\prime}, K^{\prime}\right)$ of $(S, K)$ extends to an admissible covering over $S^{\prime}$, after replacing $S^{\prime}$ by an open neighborhood of a point above $s$ in a finite extension of $S^{\prime}$. This implies that $\operatorname{sp}^{\log }: \pi_{1}^{\mathrm{t}}\left(X_{\bar{K}}^{*}, D_{\bar{K}}\right) \rightarrow \pi_{1}^{\log }\left(X_{s^{\mathrm{t}}}^{*}, D_{s^{\mathrm{t}}}\right)$ is an isomorphism.

(RamG) $\Longrightarrow$ (Sing). Suppose that (Sing) does not hold. Then, by [Tam1], Lemma (5.5), (ii) $\Longrightarrow$ (i), one verifies immediately that (RamG) does not hold.

(WRamS $) \Longrightarrow$ (Sing). Suppose that (Sing) does not hold. Then, by [Tam1], Lemma (5.5), (ii) $\Longrightarrow$ (i), one verifies immediately that (WRamS) does not hold.

Proposition 4.3. $\quad$ Let $x$ be a closed point of $X^{*}$. Let $s$ be the image of $x$ in $S$ and let $p \geq 0$ be the characteristic of the residue field of $s$. Consider the following conditions:

(Sing $)_{x}$ There exist a finite extension $\left(S^{\prime}, K^{\prime}\right)$ of $(S, K)$ and a generically tame, stable covering $\left(Y_{S^{\prime}}^{*}, E_{S^{\prime}}\right)$ of $\left(X_{S^{\prime}}^{*}, D_{S^{\prime}}\right)$ over $S^{\prime}$, such that the image of $\left(Y_{S^{\prime}}^{*}\right)^{\text {non-sm }}$ in $X^{*}$ contains $x$.

(Vert $)_{x}$ There exist a finite extension $\left(S^{\prime}, K^{\prime}\right)$ of $(S, K)$ and a generically tame, stable covering $\left(Y_{S^{\prime}}^{*}, E_{S^{\prime}}\right)$ of $\left(X_{S^{\prime}}^{*}, D_{S^{\prime}}\right)$ over $S^{\prime}$, such that the image of $V_{Y_{S^{\prime}}^{*}} / X_{S^{\prime}}^{*}$ in $X$ contains $x$.

$(\mathrm{RamS1})_{x}$ (resp. $\left.(\mathrm{WRamS1})_{x}\right)$ There exist a finite extension $\left(S^{\prime}, K^{\prime}\right)$ of $(S, K)$, a generically tame, stable coverings $\left(Y_{S^{\prime}}^{*}, E_{S^{\prime}}\right) \rightarrow\left(\left(X^{\prime}\right)_{S^{\prime}}^{*},\left(D^{\prime}\right)_{S^{\prime}}\right) \rightarrow\left(X_{S^{\prime}}^{*}, D_{S^{\prime}}\right)$ over $S^{\prime}$, a point $s^{\prime} \in S^{\prime}$ above $s$, a point $\overline{x^{\prime}} \in\left(X^{\prime}\right) \frac{*}{s^{\prime}}-\left(D^{\prime}\right) \overline{s^{\prime}}\left(\right.$ resp. $\left.\overline{x^{\prime}} \in\left(X^{\prime}\right) \frac{*}{s^{\prime}}\right)$ 
above $x$, an irreducible component $Z^{\prime}=\left\{z^{\prime}\right\}^{\mathrm{cl}}$ of $\operatorname{Spec}\left(\widehat{\mathcal{O}_{\left(X^{\prime}\right)} \overline{\frac{*}{\prime}}^{\prime}, \overline{x^{\prime}}}\right)$, and a point $w$ of the fiber $Y_{S^{\prime}}^{*} \underset{\left(X^{\prime}\right)^{*}}{\times} z^{\prime}$, such that the natural morphism from the integral closure $W^{\mathrm{s}}$ of $Z^{\prime}$ in $\boldsymbol{k}(w)^{\mathrm{s}}$ to $Z^{\prime}$ is ramified (resp. wildly ramified). Here, $\boldsymbol{k}(w)^{\mathrm{s}} / \boldsymbol{k}\left(z^{\prime}\right)$ denotes the maximal separable subextension of $\boldsymbol{k}(w) / \boldsymbol{k}\left(z^{\prime}\right)$.

$(\text { RamS2 })_{x}$ (resp. $\left.(\mathrm{WRamS2})_{x}\right)$ There exist a finite extension $\left(S^{\prime}, K^{\prime}\right)$ of $(S, K)$, generically tame, stable coverings $\left(Y_{S^{\prime}}^{*}, E_{S^{\prime}}\right) \rightarrow\left(\left(X^{\prime}\right)_{S^{\prime}}^{*},\left(D^{\prime}\right)_{S^{\prime}}\right) \rightarrow\left(X_{S^{\prime}}^{*}, D_{S^{\prime}}\right)$ over $S^{\prime}$, a point $s^{\prime} \in S^{\prime}$ above $s$, a point $\overline{x^{\prime}} \in\left(X^{\prime}\right) \frac{*}{s^{\prime}}-\left(D^{\prime}\right) \overline{s^{\prime}}\left(\right.$ resp. $\overline{x^{\prime}} \in$ $\left.\left(X^{\prime}\right) \frac{*}{s^{\prime}}\right)$ above $x$, such that, for each irreducible component $Z^{\prime}=\left\{z^{\prime}\right\}^{\mathrm{cl}}$ of $\operatorname{Spec}\left(\widehat{\mathcal{O}_{\left(X^{\prime}\right) \frac{*}{s^{\prime}}}, \bar{x}^{\prime}}\right)$ and each point $w$ of the fiber $Y_{S^{\prime}}^{*} \underset{X^{*}}{\times} z^{\prime}$, the natural morphism from the integral closure $W^{\mathrm{s}}$ of $Z^{\prime}$ in $\boldsymbol{k}(w)^{\mathrm{s}}$ to $Z^{\prime}$ is ramified (resp. wildly ramified).

(RamG1) $)_{x}$ There exist a finite extension $\left(S^{\prime}, K^{\prime}\right)$ of $(S, K)$, a generically tame, stable covering $\left(Y_{S^{\prime}}^{*}, E_{S^{\prime}}\right)$ of $\left(X_{S^{\prime}}^{*}, D_{S^{\prime}}\right)$ over $S^{\prime}$, a point $\bar{x} \in X_{\bar{s}}^{*}$ above $x$, an irreducible component $Z=\{z\}^{\mathrm{cl}}$ of $\operatorname{Spec}\left(\widehat{\mathcal{O}_{X_{\bar{s}}^{*}, \bar{x}}}\right)$, and a point $w$ of the fiber $Y_{S^{\prime}}^{*} \underset{X^{*}}{\times} z$, such that $\boldsymbol{k}(w) / \boldsymbol{k}(z)$ is inseparable.

(RamG2) $)_{x}$ There exist a finite extension $\left(S^{\prime}, K^{\prime}\right)$ of $(S, K)$, a generically tame, stable covering $\left(Y_{S^{\prime}}^{*}, E_{S^{\prime}}\right)$ of $\left(X_{S^{\prime}}^{*}, D_{S^{\prime}}\right)$ over $S^{\prime}$, such that, for each point $\bar{x} \in X_{\bar{s}}^{*}$ above $x$, each irreducible component $Z=\{z\}^{\mathrm{cl}}$ of $\operatorname{Spec}\left(\widehat{\mathcal{O}_{X_{s}^{*}}, \bar{x}}\right)$, and each point $w$ of the fiber $Y_{S^{\prime}}^{*} \underset{X^{*}}{\times} z, \boldsymbol{k}(w) / \boldsymbol{k}(z)$ is inseparable.

Then:

(i) $(\mathrm{RamS} 1)_{x}$ and $(\mathrm{RamS} 2)_{x}$ are equivalent to $(\mathrm{Sing})_{x}$.

(ii) If, moreover, $p>0$, then we have the following implications:

$$
\begin{array}{cc}
(\mathrm{Sing})_{x} \Longleftarrow(\mathrm{WRamS} 1)_{x} & \Longrightarrow \\
\Uparrow & (\mathrm{RamG} 1)_{x} \\
\Uparrow & \Uparrow \\
(\mathrm{Vert})_{x} \Longleftarrow(\mathrm{WRamS2})_{x} & (\mathrm{RamG} 2)_{x}
\end{array}
$$

Remark 4.4. (i) "Vert" stands for "vertical".

(ii) Neither $(\operatorname{RamG} 1)_{x}$ nor $(\text { RamG2) })_{x}$ implies (Sing) $)_{x}$. For this, see Remark 5.1 below.

Proof. Replacing $\left(X^{*}, D\right)$ by its base change to $\operatorname{Spec}\left(\widehat{\mathcal{O}_{S, s}}\right)$, we may assume that $S$ is the spectrum of a complete discrete valuation ring $R$.

(i) $(\text { Sing })_{x} \Longrightarrow(\text { RamS2 })_{x}$. This follows from a similar argument to that applied in the proof of (Sing) $\Longrightarrow($ RamS) in Proposition 4.1(i). 
$(\operatorname{RamS} 2)_{x} \Longrightarrow(\operatorname{RamS} 1)_{x}$. Clear.

$(\operatorname{RamS1})_{x} \Longrightarrow(\text { Sing })_{x}$. Assume that $(\text { RamS1 })_{x}$ holds. First, replacing $X_{S^{\prime}}^{*}$ by $\left(X^{\prime}\right)_{S^{\prime}}^{*}$ and $Y_{S^{\prime}}^{*}$ by its Galois closure, we may assume that $\left(X^{\prime}\right)_{S^{\prime}}^{*}=X_{S^{\prime}}^{*}$ and that $Y_{S^{\prime}}^{*}$ is Galois over $X_{S^{\prime}}^{*}$. Thus, we shall write $\bar{x}, Z$ and $z$ instead of $\overline{x^{\prime}}, Z^{\prime}$ and $z^{\prime}$, respectively. Then, replacing $X_{S^{\prime}}^{*}$ and $Y_{S^{\prime}}^{*}$ by the subcoverings of $Y_{S^{\prime}}^{*} / X_{S^{\prime}}^{*}$ that correspond to the inertia subgroup and the decomposition subgroup, respectively, at $w$, we may assume that $\boldsymbol{k}(w)=\boldsymbol{k}(w)^{\mathrm{s}}$ holds, i.e., $\boldsymbol{k}(w)$ is separable over $\boldsymbol{k}(z)$. Thus, we shall write $W$ instead of $W^{\mathrm{s}}$. Now, suppose that $(\operatorname{Sing})_{x}$ does not hold. Then we have $Z=\operatorname{Spec}\left(\widehat{\mathcal{O}_{X \frac{*}{s^{\prime}}} \bar{x}}\right)$ and $W=\operatorname{Spec}\left(\widehat{\mathcal{O}_{Y \frac{*}{s^{\prime}}}}\right)$ for some point $\bar{y} \in Y_{\overline{s^{\prime}}}^{*}$ above $\bar{x}$. Then, by the purity of the branched locus, the above separability assumption and the condition $\bar{x} \notin D \overline{\bar{s}^{\prime}}$ imply that $Y_{S^{\prime}}^{*} \rightarrow X_{S^{\prime}}^{*}$ is étale at (the image of) $\bar{y}$. This implies that $W \rightarrow Z$ is étale. (This also gives an alternative (local) proof of (RamS) $\Longrightarrow$ (Sing) in Proposition 4.1(i).)

(ii) $(\text { Vert })_{x} \Longrightarrow(\text { Sing })_{x}$. This is immediate, since each vertical component meets at least one other component.

$(\mathrm{WRamS} 1)_{x} \Longrightarrow(\mathrm{Sing})_{x}$. Assume that $(\mathrm{WRamS1})_{x}$ holds. First, replacing $X_{S^{\prime}}^{*}$ by $\left(X^{\prime}\right)_{S^{\prime}}^{*}$ and $Y_{S^{\prime}}^{*}$ by its Galois closure, we may assume that $\left(X^{\prime}\right)_{S^{\prime}}^{*}=X_{S^{\prime}}^{*}$ and that $Y_{S^{\prime}}^{*}$ is Galois over $X_{S^{\prime}}^{*}$. Thus, we shall write $\bar{x}, Z$ and $z$ instead of $\overline{x^{\prime}}, Z^{\prime}$ and $z^{\prime}$, respectively. Then, replacing $X_{S^{\prime}}^{*}$ and $Y_{S^{\prime}}^{*}$ by the subcoverings of $Y_{S^{\prime}}^{*} / X_{S^{\prime}}^{*}$ of $X_{S^{\prime}}^{*}$ that correspond to the inertia subgroup and the decomposition subgroup, respectively, at $w$, we may assume that $\boldsymbol{k}(w)=\boldsymbol{k}(w)^{\mathrm{s}}$ holds, i.e., $\boldsymbol{k}(w)$ is separable over $\boldsymbol{k}(z)$. Thus, we shall write $W$ instead of $W^{\mathrm{s}}$. Now, suppose that $(\operatorname{Sing})_{x}$ does not hold. Then we have $Z=\operatorname{Spec}\left(\widehat{\mathcal{O}_{X \frac{*}{s^{\prime}}}, \bar{x}}\right)$ and $W=\operatorname{Spec}\left(\widehat{\mathcal{O}_{Y s^{\prime}} \bar{y}}\right)$ for some point $\bar{y} \in Y_{\bar{s}^{\prime}}^{*}$ above $\bar{x}$. Then, by the purity of the branched locus and Lemma 2.1(i)(ii), the above separability assumption implies that any ramification of $Y_{S^{\prime}}^{*}$ over $X_{S^{\prime}}^{*}$ at (the image of) $\bar{y}$ is at most tame ramification along $D_{S^{\prime}}$. Thus, any ramification of $W$ over $Z$ is at most tame ramification along $D \overline{\bar{s}^{\prime}}$. In particular, $W \rightarrow Z$ is at most tamely ramified. (This also gives an alternative (local) proof of (WRamS) $\Longrightarrow$ (Sing) in Proposition 4.1(ii).)

$(\mathrm{WRamS2})_{x} \Longrightarrow(\text { Vert })_{x}$. By a standard argument, we may reduce the problem to the case where $S$ is the spectrum of a complete discrete valuation ring with algebraically closed field. Assume that (WRamS2) $)_{x}$ holds. First, replacing $S$ by a suitable finite extension $S^{\prime \prime}$ of $S^{\prime}, X^{*}$ by $\left(X^{\prime}\right)_{S^{\prime \prime}}^{*}$ and $Y_{S^{\prime}}^{*}$ by the stable model over $S^{\prime \prime}$ of its Galois closure, we may assume that $\left(X^{\prime}\right)_{S^{\prime}}^{*}=X^{*}$ and that $Y^{*}$ is Galois with Galois group $G$ over $X^{*}$. Thus, we may write $s$ 
and $x$ instead of $\overline{s^{\prime}}$ and $\overline{x^{\prime}}$, respectively. Now, suppose that (Vert) $)_{x}$ does not hold. Then $Y^{*} \rightarrow X^{*}$ is finite at $x$. Replacing $X^{*}$ by the covering of $X^{*}$ corresponding to the decomposition subgroup at $x$, we may assume that there exists a unique point $y \in Y_{s}^{*}$ above $x$. Let $a$ (resp. $b$ ) denote the number of irreducible components of $\operatorname{Spec}\left(\widehat{\mathcal{O}_{X_{s}^{*}, x}}\right)\left(\operatorname{resp} . \operatorname{Spec}\left(\widehat{\mathcal{O}_{Y_{s}^{*}, y}}\right)\right)$. Thus, $(a, b)$ is one of the following: $(1,1),(1,2),(2,2)$. We define $Z_{i}=\left\{z_{i}\right\}^{\mathrm{cl}}(i=1, \ldots, a)$ and $W_{j}=\left\{w_{j}\right\}^{\mathrm{cl}}(j=1, \ldots, b)$ to be the irreducible components of $\operatorname{Spec}\left(\widehat{\mathcal{O}_{X_{s}^{*}, x}}\right)$ and $\operatorname{Spec}\left(\widehat{\mathcal{O}_{Y_{s}^{*}}, y}\right)$, respectively. We may assume that $w_{1}$ is above $z_{1}$ and $w_{b}$ is above $z_{a}$. For each $j=1, \ldots, b$, we denote the decomposition group, the inertia group, and the wild inertia group at $w_{j}$ by $D_{j}, I_{j}$, and $I_{j}^{\mathrm{w}}$, respectively.

First, assume $(a, b)=(1,1)$. In this case, as in the above proof of (WRam $\mathrm{S} 1)_{x} \Longrightarrow(\mathrm{Sing})_{x}$, we conclude that $W_{1}^{\mathrm{s}} \rightarrow Z_{1}$ is at most tamely ramified. This contradicts our choice of $Y^{*}$.

Next, assume $(a, b)=(2,2)$. In this case, we have $D_{1}=D_{2}=G$. Since both $Y_{s}^{*}$ and $X_{s}^{*}$ are reduced, we have $I_{j}=I_{j}^{\mathrm{w}}$ for each $j=1,2$. By our choice of $Y^{*}$ (i.e., by the wild ramification condition in (WRamS2) $\left.)_{x}\right), G / I_{j}=$ $D_{j} / I_{j}$ has order divisible by $p$ for each $j=1,2$. By Lemma 2.1(iii), $G / I_{1} I_{2}$ is of order prime to $p$. Thus, replacing $X^{*}$ by the covering corresponding to $I_{1} I_{2} \subset G$, we may assume $I_{1} I_{2}=G$. Since both $I_{1}$ and $I_{2}$ are normal $p$ subgroups of $G=D_{1}=D_{2}$, one verifies easily that $G=I_{1} I_{2}$ is a $p$-group. Now, observe that we have a decomposition $G /\left(I_{1} \cap I_{2}\right) \stackrel{\sim}{\rightarrow} G / I_{1} \times G / I_{2}$. As $G / I_{j}$ is a nontrivial $p$-group, we may choose $G / I_{j} \rightarrow \mathbb{Z} / p \mathbb{Z}$. Then, from this decomposition, we obtain $G \rightarrow G / I_{1} \times G / I_{2} \rightarrow(\mathbb{Z} / p \mathbb{Z})^{2}$. Now, consider the subcoverings $\left(X^{\prime \prime \prime}\right)^{*} \rightarrow\left(X^{\prime \prime}\right)^{*} \rightarrow X^{*}$ of $Y^{*} \rightarrow X^{*}$ that correspond to $G \rightarrow$ $(\mathbb{Z} / p \mathbb{Z})^{2} \stackrel{\operatorname{sum}}{\rightarrow} \mathbb{Z} / p \mathbb{Z}$. We denote by $z_{j}^{\prime \prime}$ the image of $w_{j}$ in $\left(X^{\prime \prime}\right)_{s}$ for each $j=1,2$. Then, by construction, the $\mathbb{Z} / p \mathbb{Z}$-covering $\left(X^{\prime \prime \prime}\right)^{*} \rightarrow\left(X^{\prime \prime}\right)^{*}$ is unramified over both $z_{1}^{\prime \prime}$ and $z_{2}^{\prime \prime}$. However, by Lemma 2.1(iii), such a covering must be of order prime to $p$. This is absurd.

Finally, assume $(a, b)=(1,2)$. In this case, $\left(G: D_{1}\right)=2$. In particular, $D_{1}$ is normal in $G$. Since $D_{2}$ is conjugate to $D_{1}$, we have $D_{1}=D_{2}$. Thus, replacing $X_{S^{\prime}}^{*}$ by the double covering corresponding to $D_{1}=D_{2} \subset G$, we can reduce the problem to the previous case $(a, b)=(2,2)$.

$(\mathrm{WRamS} 2)_{x} \Longrightarrow(\mathrm{WRamS} 1)_{x}$. Clear.

$(\text { RamG2 })_{x} \Longrightarrow(\text { RamG1 })_{x}$. Clear.

$(\mathrm{WRamS1})_{x} \Longrightarrow(\operatorname{RamG1})_{x}$. Suppose that (RamG1) $)_{x}$ does not hold. Then Lemma 2.1(i)(ii)(iii), together with the purity of the branched locus, implies that each $W^{\mathrm{s}} \rightarrow Z^{\prime}$ as in the statement of (WRamS1) $)_{x}$ must be at most tamely ramified. 


\section{$\S 5 . \quad$ Proof of the Main Theorem}

In this $\S$, we give a proof of Theorem 0.2 .

(i) This follows from Proposition 2.2(iii).

(ii) For the first assertion, we may easily reduce the problem to the case that $k$ is algebraically closed and $S$ is proper and smooth. Then this follows from Theorem 3.14, together with Proposition 4.1, (Sp1) $\Longrightarrow$ (Sing). For the second assertion, let $k_{0}$ be an algebraically closed field of characteristic $p, S_{0}$ a proper, smooth, connected curve over $k_{0}$, and $K_{0}$ the function field of $S_{0}$. Take any generically smooth, stable marked curve $\left(X_{0}^{*}, D_{0}\right)$ over $S_{0}$ that is not $k_{0}$-isotrivial. Next, let $k$ be another algebraically closed field that contains $k_{0}$ but does not coincide with $k_{0}$. Set $S \stackrel{\text { def }}{=} S_{0} \times_{k_{0}} k$ and $\left(X^{*}, D\right) \stackrel{\text { def }}{=}\left(X^{*} \times{ }_{S_{0}} S, D \times_{S_{0}} S\right)$. Then $\left(X^{*}, D\right)$ is a generically smooth, stable marked curve over $S$ that is not $k$-isotrivial. Thus, the assertion in (II) does not hold for any $s \in S(k)-S_{0}\left(k_{0}\right)$. More precisely, take any such point $s$. The image of $s$ in $S_{0}$ is the generic point of $S_{0}$. Then, by means of the fact that the natural map $\pi_{1}^{\mathrm{t}}\left(X_{s}^{*}, D_{s}\right) \rightarrow \pi_{1}^{\mathrm{t}}\left(\left(X_{0}\right)_{\overline{K_{0}}},\left(D_{0}\right)_{\overline{K_{0}}}\right)$ is an isomorphism, one verifies immediately that the assertion in (II) does not hold for $s$.

(iii) Just as in the case of (ii), this follows from Theorem 3.13, together with Proposition 4.1, (Sp1) $\Longrightarrow$ (Sing).

(iv) Replacing $\left(X^{*}, D\right)$ by a suitable covering, we may assume that $g>0$ holds. Then the first assertion follows from (2.5), together with Proposition $4.1,(\mathrm{Jac} 1) \Longrightarrow$ (Sing). For the second assertion, let $k_{0}$ be an algebraically closed field of characteristic $p>0$, set $S_{0} \stackrel{\text { def }}{=} \operatorname{Spec}\left(W\left(k_{0}\right)\right)$, and take any generically smooth, stable marked curve $\left(X_{0}^{*}, D_{0}\right)$ over $S_{0}$. Next, let $k$ be another algebraically closed field that contains $k_{0}$ but does not coincide with $k_{0}$. Set $S \stackrel{\text { def }}{=} \operatorname{Spec}(W(k))$ and $\left(X^{*}, D\right) \stackrel{\text { def }}{=}\left(X^{*} \times{ }_{S_{0}} S, D \times_{S_{0}} S\right)$. We set $X_{0} \stackrel{\text { def }}{=} X_{0}^{*}-D_{0}$ (resp. $\left.X \stackrel{\text { def }}{=} X^{*}-D\right)$, and denote by $K_{0}($ resp. $K)$ the function field of $S_{0}$ (resp. $S$ ). Then $\left(X^{*}, D\right)$ is a generically smooth, stable marked curve over $S$. Now, by means of the fact that $\pi_{1}\left((X)_{\bar{K}}\right) \stackrel{\sim}{\rightarrow} \pi_{1}\left(\left(X_{0}\right)_{\overline{K_{0}}}\right)$ holds, one verifies easily that the assertion of (III) does not hold for any $x \in X_{k}^{*}(k)-\left(X_{0}^{*}\right)_{k_{0}}\left(k_{0}\right)$.

Remark 5.1. Note that the above argument also shows that (RamG1) (or even $(\text { RamG2) })_{x}$ ) does not imply $(\mathrm{Sing})_{x}$ in Proposition 4.3. Indeed, let the notations and assumptions be as in the above proof of (iv), and take $x \in$ $X_{k}^{*}(k)-\left(X_{0}^{*}\right)_{k_{0}}\left(k_{0}\right)$. Then we have already seen that $(\mathrm{Sing})_{x}$ does not hold. On 
the other hand, assume, moreover, that $\left(X_{0}^{*}, D_{0}\right)$ is a smooth marked curve over $S_{0}$ (i.e., $X_{0}^{*}$ is smooth over $S_{0}$ ). In particular, $X_{\bar{s}}^{*}$ is irreducible. Now, by the first assertion of Theorem 0.2(iv) and Proposition 4.1(ii), (Sing) $\Longrightarrow$ (RamG), one verifies immediately that $(\text { RamG2) })_{x}$ (hence $(\text { RamG1) })_{x}$ ) holds.

(v) We can easily reduce the problem to the case where $S$ is the spectrum of a complete discrete valuation ring $R$ of mixed characteristic $(0, p)$ and with residue field algebraic over $\mathbb{F}_{p}$. Moreover, replacing $R$ by the completion of the strict henselization, we may assume that the residue field is an algebraic closure of $\mathbb{F}_{p}$. Let $K$ and $k$ denote the field of fractions and the residue field, respectively, of $R$.

We shall prove that condition $(\mathrm{WRamS2})_{x}$ holds. First, choose an admissible covering $\left(\left(X^{\prime}\right)_{S^{\prime}}^{*}, D_{S^{\prime}}^{\prime}\right)$ of $\left(X_{S^{\prime}}^{*}, D_{S^{\prime}}\right)$ over some finite tame extension $S^{\prime}$ of $S$, such that $\left(X^{\prime}\right)_{\frac{*}{s}}^{*}$ is "sturdy", i.e., for every irreducible component $\left(Z^{\prime}\right)^{*}$ of $\left(X^{\prime}\right) \frac{*}{s}$, the normalization $\left(\left(Z^{\prime}\right)^{*}\right)^{\sim}$ has genus $\geq 2$ (cf. [M2], Definition 1.1). This can be done by means of Remark 3.3. (See also [M2], Lemma 2.9.) For simplicity, replacing $\left(X^{*}, D\right)$ by $\left(\left(X^{\prime}\right)_{S^{\prime}}^{*}, D_{S^{\prime}}^{\prime}\right)$, we shall assume that $X_{s}^{*}$ is sturdy.

Now, let $Z=\{z\}^{\mathrm{cl}}$ be an irreducible component of $\operatorname{Spec}\left(\widehat{\mathcal{O}_{X_{s}^{*}, x}}\right)$. (We have two choices (resp. only one choice) of $Z$, if $x$ is (resp. is not) a node of $X_{s}^{*}$.) We have a natural morphism $Z \rightarrow X^{*}$, and we denote by $z_{\mathrm{gl}}$ the image of $z$ in $X^{*}$, which is the generic point of an irreducible component $Z^{*}$ of $X_{s}$. ("gl" stands for "global".)

One important point of our proof is the introduction of the family

$$
\pi: \mathcal{X} \stackrel{\text { def }}{=}\left(X^{*} \times_{S} X^{*},\left(D \times{ }_{S} X^{*}\right) \cup \Delta\left(X^{*}\right)\right) \rightarrow X^{*},
$$

where $\Delta$ is the diagonal and $\pi$ is the second projection. $\pi$ itself is not always a stable marked curve over $X^{*}$, but $\left.\pi\right|_{U}: \mathcal{X}_{U} \rightarrow U$ is a stable marked curve, where $X \stackrel{\text { def }}{=} X^{*}-D$ and $U \stackrel{\text { def }}{=} X-\left(X^{*}\right)^{\text {non-sm }}$. (As $X^{*}$ is generically smooth, we have $X_{K} \subset U$.) Let $A$ denote the completion of the local ring $\mathcal{O}_{X^{*}, z_{\mathrm{gl}}}$. This is a complete discrete valuation ring of mixed characteristic $(0, p)$. We set $T \stackrel{\text { def }}{=} \operatorname{Spec}(A)$. We denote the closed point of $T$ again by $z_{\mathrm{gl}}$, and the generic point of $T$ by $\xi$. We have a natural map $\iota: T \rightarrow X^{*}$. We denote by $\xi_{\mathrm{gl}}$ the image of $\xi$ in $X^{*}$. Namely, $\xi_{\mathrm{gl}}$ is the generic point of $X_{K}^{*}$ (or, of $X^{*}$ ).

Since $\iota$ factors through $U \subset X^{*}, \pi_{T}: \mathcal{X}_{T} \rightarrow T$ is a stable marked curve. More concretely, we have $\mathcal{X}_{T}=\left(X^{*} \times_{S} T,\left(D \times_{S} T\right) \cup \Gamma_{\iota}\right)$, where $\Gamma$ denotes the graph. $\pi_{\xi}: \mathcal{X}_{\xi} \rightarrow \xi$ is a smooth, stable marked curve over $\xi$, while $\pi_{z_{\mathrm{g} 1}}$ : $\mathcal{X}_{z_{\mathrm{g} 1}} \rightarrow z_{\mathrm{gl}}$ is a stable marked curve over $z_{\mathrm{gl}}$. More concretely, we have $\mathcal{X}_{z_{\mathrm{gl}}}=$ 
$\left(X_{s}^{*} \times_{s} z_{\mathrm{gl}},\left(D \times_{s} z_{\mathrm{gl}}\right) \cup \Gamma_{\iota_{z_{\mathrm{gl}}}}\right)$, where $\iota_{z_{\mathrm{gl}}}$ denotes the natural morphism $z_{\mathrm{gl}} \rightarrow X_{s}^{*}$. Note that $\mathcal{Z} \stackrel{\text { def }}{=} Z_{z_{\mathrm{g} 1}}^{*}-\left(D_{z_{\mathrm{gl}}} \cup \Gamma_{\iota_{z_{\mathrm{g} 1}}}\right)$ is a connected component of $\left(X_{z_{\mathrm{gl}}}^{*}\right)^{\mathrm{sm}}-$ $\left(D_{z_{\mathrm{g} 1}} \cup \Gamma_{\iota_{z_{\mathrm{g} 1}}}\right)$.

We have the following commutative diagram of profinite groups in which all seven rows are exact:

$$
\begin{aligned}
& 1 \rightarrow \pi_{1}\left(\mathcal{X}_{\overline{\xi_{\mathrm{g} 1}}}\right) \quad \rightarrow \pi_{1}\left(\mathcal{X}_{X_{K}}\right) \rightarrow \pi_{1}\left(X_{K}\right) \rightarrow 1
\end{aligned}
$$

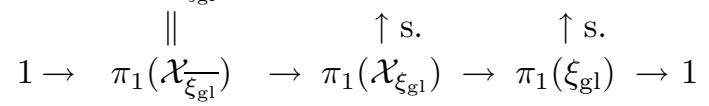

$$
\begin{aligned}
& \| \quad \uparrow \quad \uparrow \\
& 1 \rightarrow \pi_{1}\left(\mathcal{X}_{\bar{\xi}}\right) \quad \rightarrow \quad \pi_{1}\left(\mathcal{X}_{\xi}\right) \rightarrow \pi_{1}(\xi) \rightarrow 1 \\
& \downarrow \text { s. } \quad \downarrow \text { s. } \downarrow \text { s. } \\
& 1 \rightarrow \pi_{1}^{\log }\left(\mathcal{X}_{\left(z_{\mathrm{g} l}\right)^{\mathrm{t}}}\right) \rightarrow \pi_{1}^{\log }\left(\mathcal{X}_{z_{\mathrm{gl}}}\right) \rightarrow \pi_{1}^{\log }\left(z_{\mathrm{gl}}\right) \rightarrow 1 \\
& \cup \quad \cup \quad \| \\
& 1 \rightarrow \pi_{1}^{\mathrm{t}}\left(\mathcal{Z}_{\overline{z_{\mathrm{gl}}}}\right) \quad \rightarrow \quad \mathbf{D}_{\mathcal{Z}} \quad \rightarrow \pi_{1}^{\log }\left(z_{\mathrm{gl}}\right) \rightarrow 1 \\
& \| \quad \downarrow \text { s. } \quad \downarrow \mathrm{s} . \\
& \left.1 \rightarrow \pi_{1}^{\mathrm{t}}\left(\mathcal{Z}_{\overline{z_{\mathrm{gl}}}}\right)\right) \quad \rightarrow \quad \pi_{1}^{\mathrm{t}}(\mathcal{Z}) \quad \rightarrow \pi_{1}\left(z_{\mathrm{gl}}\right) \rightarrow 1
\end{aligned}
$$

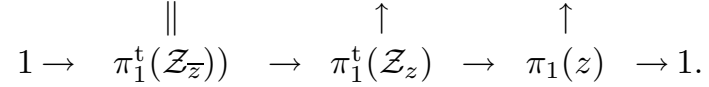

Here, "s." stands for "surjective", and "D" (resp. "I") denotes the decomposition (resp. inertia) group . Note that the exactness of the first row requires the fact that $K$ is of characteristic 0 .

For simplicity, for each $i=1, \ldots, 7$, write

$$
1 \rightarrow \Delta_{i} \rightarrow \Pi_{i} \rightarrow \Gamma_{i} \rightarrow 1
$$

for the $i$-th row of the above diagram,

$$
\rho_{i}: \Gamma_{i} \rightarrow \operatorname{Out}\left(\Delta_{i}\right)
$$

for the outer representation associated with this exact sequence, and $\operatorname{Im}_{i}$ for $\operatorname{Im}\left(\rho_{i}\right)$. Then, by Remark 2.3 and Lemma 2.4, we have

$$
\mathrm{Im}_{1}=\mathrm{Im}_{2} \hookleftarrow \operatorname{Im}_{3} \rightarrow \operatorname{Im}_{4} \rightarrow \operatorname{Im}_{5}=\operatorname{Im}_{6} \hookleftarrow \operatorname{Im}_{7} .
$$

Here, the only nontrivial part is $\operatorname{Im}_{4} \rightarrow \operatorname{Im}_{5}$, where we have used the following (cf. Remark 2.3(ii)):

Lemma 5.2. $\quad \Delta_{5}$ is normally terminal in $\Delta_{4}$ (in the sense of [M4], Definition 0.1 (iii)), i.e., the normalizer of $\Delta_{5}$ in $\Delta_{4}$ coincides with $\Delta_{5}$. 
Proof. (See also [M6].) First note that $\Delta_{5}=\pi_{1}^{\mathrm{t}}\left(\mathcal{Z}_{\overline{z_{g 1} 1}}\right)$ is the decomposition group of $Z_{\bar{z}_{\mathrm{g} 1}^{*}}^{*}$ in $\Delta_{4}=\pi_{1}^{\log }\left(\mathcal{X}_{\left(z_{\mathrm{g} 1}\right)^{\mathrm{t}}}\right)$. More precisely, $\Delta_{5}$ is the decomposition group in $\Delta_{4}$ of an irreducible component $\left(Z^{*}\right)^{\sim}$ above $Z_{z_{\mathrm{g} 1}}^{*}$ of the "log universal covering" $\left(\left(\mathcal{X}_{z_{\mathrm{gl}}}\right)^{\sim}\right)^{\log }$ of $\mathcal{X}_{z_{\mathrm{g} 1}}^{\log }$. Thus, $\Delta_{5}$ is normally terminal in $\Delta_{4}$ if and only if, for each irreducible component $\left(\left(Z^{*}\right)^{\sim}\right)^{\prime}$ above $Z_{z_{\mathrm{g} 1}}^{*}$ of $\left(\mathcal{X}_{z_{\mathrm{gl}}}\right)^{\sim}$ that is distinct from $\left(Z^{*}\right)^{\sim}$, the decomposition group $\Delta_{5}^{\prime}$ of $\left(\left(Z^{*}\right)^{\sim}\right)^{\prime}$ does not coincide with that of $\left(Z^{*}\right)^{\sim}$, i.e., $\Delta_{5}$.

Now, since $\left(Z^{*}\right)^{\sim} \neq\left(\left(Z^{*}\right)^{\sim}\right)^{\prime}$, there exists a finite $(\log )$ covering $\mathcal{X}_{1}$ of $\mathcal{X}_{z_{\mathrm{g} 1}}$, such that the image $Z_{1}^{*}$ of $\left(Z^{*}\right)^{\sim}$ in $\mathcal{X}_{1}$ is distinct from the image $\left(Z_{1}^{*}\right)^{\prime}$ of $\left(\left(Z^{*}\right)^{\sim}\right)^{\prime}$ in $\mathcal{X}_{1}$. Replacing $\mathcal{X}_{1}$ by a suitable covering if necessary, we may assume that the genera of the normalizations of $Z_{1}^{*}$ and $\left(Z_{1}^{*}\right)^{\prime}$ are positive.

We denote by $\left(\Delta_{5}\right)_{1}\left(\operatorname{resp} .\left(\Delta_{5}^{\prime}\right)_{1}\right)$ the intersection of $\Delta_{5}$ (resp. $\left.\Delta_{5}^{\prime}\right)$ and $\left(\Delta_{4}\right)_{1} \stackrel{\text { def }}{=} \pi_{1}^{\log }\left(\left(\mathcal{X}_{1}\right)_{z_{\mathrm{gl}}^{\mathrm{t}}}\right)$ in $\Delta_{4}=\pi_{1}^{\log }\left(\mathcal{X}_{z_{\mathrm{gl}}^{\mathrm{t}}}\right)$. We denote by $\left(\left(\Delta_{4}\right)_{1}\right)^{\text {ét }}$ the maximal quotient of $\left(\Delta_{4}\right)_{1}$ among those corresponding to étale coverings of $\left(\mathcal{X}_{1}\right)_{z_{\mathrm{g} 1}^{\mathrm{t}}}$. Now, one verifies easily that the images of $\left(\Delta_{5}\right)_{1}$ and $\left(\Delta_{5}^{\prime}\right)_{1}$ in $\left(\left(\left(\Delta_{4}\right)_{1}\right)^{\text {ét }}\right)^{\text {ab }}$ are nontrivial and distinct from each other. Thus, in particular, we have $\Delta_{5} \neq \Delta_{5}^{\prime}$, as desired.

In particular, we have

$$
\begin{aligned}
& \operatorname{Im}_{1} \hookleftarrow \operatorname{Im}_{3} \rightarrow \operatorname{Im}_{6} \supset \operatorname{Im}_{7} \\
& \uparrow \begin{array}{cccc}
\uparrow & \uparrow & \uparrow & \uparrow
\end{array} \\
& \pi_{1}\left(X_{K}\right) \leftarrow \pi_{1}(\xi) \rightarrow \pi_{1}\left(z_{\mathrm{gl}}\right) \leftarrow \pi_{1}(z),
\end{aligned}
$$

where all the vertical arrows are surjective. Now, let $\mathbf{I}_{z_{\mathrm{gl}}}$ be the inertia subgroup of $\pi_{1}(\xi)$, and denote by $\overline{\mathbf{D}}_{z_{\mathrm{g} 1}}$ and $\overline{\mathbf{I}}_{z_{\mathrm{g} 1}}$ the images of $\pi_{1}(\xi)$ and $\mathbf{I}_{z_{\mathrm{g} 1}}$, respectively, in $\pi_{1}\left(X_{K}\right)$. The injectivity of $\operatorname{Im}_{3} \rightarrow \operatorname{Im}_{1}$ implies that $\pi_{1}(\xi) \rightarrow \operatorname{Im}_{3}$ factors through $\overline{\mathbf{D}}_{z_{\mathrm{g} 1}}$. Thus, the surjectivity of $\operatorname{Im}_{3} \rightarrow \operatorname{Im}_{6}$ implies that $\pi_{1}(\xi) \rightarrow$ $\pi_{1}\left(z_{\mathrm{gl}}\right) \rightarrow \operatorname{Im}_{6}$ factors through $\overline{\mathbf{D}}_{z_{\mathrm{gl}} \mathrm{l}}$, and through $\overline{\mathbf{D}}_{z_{\mathrm{gl}}} / \overline{\mathbf{I}}_{z_{\mathrm{gl}}}$ since $\mathbf{I}_{z_{\mathrm{gl}}}$ is just the kernel of $\pi_{1}(\xi) \rightarrow \pi_{1}\left(z_{\mathrm{gl}}\right)$. In particular, we obtain:

$$
\begin{array}{cccc}
\pi_{1}\left(z_{\mathrm{gl}}\right) & \rightarrow \overline{\mathbf{D}}_{z_{\mathrm{gl}}} / \overline{\mathbf{I}}_{z_{\mathrm{gl}}} \rightarrow & \mathrm{Im}_{6} \\
\uparrow & & \cup \\
\pi_{1}(z) & \rightarrow & & \mathrm{Im}_{7} .
\end{array}
$$

Set $\mathbf{I}_{x} \stackrel{\text { def }}{=} \pi_{1}(z)$. (Recall that $z$ is the generic point of the spectrum $Z$ of a complete discrete valuation ring with algebraically closed residue field, and that $x$ can be regarded as the closed point of $Z$.) Let $\overline{\mathbf{I}}_{x}$ denote the image of $\mathbf{I}_{x}$ in $\overline{\mathbf{D}}_{z_{\mathrm{g} 1}} / \overline{\mathbf{I}}_{z_{\mathrm{g} 1}}$. Thus, we have

$$
\mathbf{I}_{x} \rightarrow \overline{\mathbf{I}}_{x} \rightarrow \operatorname{Im}_{7}
$$


Now, by Theorem 3.13 and Proposition 4.1, (Sp1) $\Longrightarrow$ (Mon3), the image of $\mathbf{I}_{x}^{\mathrm{w}}$ in $\operatorname{Im}_{7}$ is infinite. Hence, a fortiori, the image $\overline{\mathbf{I}}_{x}^{\mathrm{w}}$ of $\mathbf{I}_{x}^{\mathrm{w}}$ in $\overline{\mathbf{I}}_{x}$ is infinite, and, in particular, nontrivial. Since the choice of $Z=\{z\}^{\text {cl }}$ is arbitrary, this implies that (WRamS2) $)_{x}$ holds. Thus, by Proposition 4.3(ii), (Vert) $)_{x}$ and (Sing) $)_{x}$ hold, as desired.

\section{Acknowledgements}

The present paper is, at least logically speaking, a mere small corollary of [Tam3]. Thus, the author would like to thank Shinichi Mochizuki, Stefan Wewers and Florian Pop very much for having taken an interest in this work in an earlier stage, which has been encouraging to his writing. The author would also like to thank Ahmed Abbes very much for helpful discussions concerning Corollary 3.19. Indeed, the idea of using [D2] is due to him. Finally, the author would like to thank Shinichi Mochizuki (again), Kazuhiro Fujiwara and Makoto Matsumoto very much for helpful discussions and informations concerning admissible coverings, log structures, and graphs of groups.

\section{References}

[AO] Abramovich, D. and Oort, F., Stable maps and Hurwitz schemes in mixed characteristics, in Advances in algebraic geometry motivated by physics (Lowell, 2000), Contemp. Math., 276, Amer. Math. Soc., 2001, 89-100.

[D1] de Jong, A. J., Smoothness, semi-stability and alterations, Inst. Hautes Études Sci. Publ. Math., 83 (1996), 51-93.

[D2] Homomorphisms of Barsotti-Tate groups and crystals in positive characteristic, Invent. Math., 134 (1998), 301-333; Erratum, Invent. Math., 138 (1999), 225.

[F] Fujiwara, K., Etale topology and the philosophy of log, in Proceedings of Algebraic Geometry Symposium (Kinosaki), 1990, 116-123. (Japanese)

[FK] Fujiwara, K. and Kato, K., Logarithmic etale topology theory, Preprint.

[GM] Grothendieck, A. and Murre, J. P., The tame fundamental group of a formal neighbourhood of a divisor with normal crossings on a scheme, Lecture Notes in Math., 208, Springer-Verlag, 1971.

[I] Illusie, L., An overview of the work of K. Fujiwara, K. Kato, and C. Nakayama on logarithmic étale cohomology, in Cohomologies $p$-adiques et applications arithmétiques, II, Astérisque, 279 (2002), 271-322.

[Ka] Kato, K., Logarithmic structures of Fontaine-Illusie, in Algebraic analysis, geometry, and number theory (Baltimore, 1988), Johns Hopkins Univ. Press, 1989, 191-224.

[KM] Katz, N. M. and Mazur, B., Arithmetic moduli of elliptic curves, Ann. of Math. Stud., 108, Princeton University Press, 1985.

[Kn] Knudsen, F. F., The projectivity of the moduli space of stable curves, II, The stacks $M_{g, n}$, Math. Scand., 52 (1983), 161-199.

[LL] Liu, Q. and Lorenzini, D., Models of curves and finite covers, Compositio Math., 118 (1999), 61-102. 
[M1] Mochizuki, S., The geometry of the compactification of the Hurwitz scheme, Publ. RIMS, Kyoto Univ., 31 (1995), 355-441.

[M2] _ The profinite Grothendieck conjecture for closed hyperbolic curves over number fields, J. Math. Sci. Univ. Tokyo, 3 (1996), 571-627.

[M3] Extending families of curves over log regular schemes, J. Reine Angew. Math., 511 (1999), 43-71.

[M4] The absolute anabelian geometry of hyperbolic curves, in Galois theory and modular forms, Dev. Math., 11, Kluwer Acad. Publ., 2004, 77-122.

[M5] - The geometry of anabelioids, Publ. RIMS, Kyoto Univ., 40 (2004), $819-881$.

[M6] Semi-graphs of anabelioids, Manuscript.

[PS] Pop, F., and Saïdi, M., On the specialization homomorphism of fundamental groups of curves in positive characteristic, in Galois groups and fundamental groups, Math. Sci. Res. Inst. Publ., 41, Cambridge Univ. Press, 2003, 107-118.

[R] Raynaud, M., Sur le groupe fondamental d'une courbe complète en caractéristique $p>0$, in Arithmetic fundamental groups and noncommutative algebra (Berkeley, 1999), Proc. Sympos. Pure Math., 70, Amer. Math. Soc., 2002, 335-351.

[Sa] Saïdi, M., On complete families of curves with a given fundamental group in positive characteristic, Preprint, arXiv:math.AG/0305120.

[SGA1] Grothendieck, A. and Raynaud, Mme. M., Revêtements Étales et groupe fondamental, Séminaire de Géométrie Algébrique du Bois Marie 1960-1961 (SGA 1), Lecture Notes in Math., 224, Springer-Verlag, 1971.

[SGA7I] Grothendieck, A., et al., Groupes de monodromie en géométrie algébrique, I, Séminaire de Géométrie Algébrique du Bois-Marie 1967-1969 (SGA 7 I), Lecture Notes in Math., 288, Springer-Verlag, 1972.

[St] Stix, J., Projective anabelian curves in positive characteristic and descent theory for log-étale covers, Dissertation (Rheinische Friedrich-Wilhelms-Universität Bonn, 2002), Bonner Math. Schriften, 354, Universität Bonn, Mathematisches Institut, 2002 .

[Tam1] Tamagawa, A., The Grothendieck conjecture for affine curves, Compositio Math., 109 (1997), 135-194.

[Tam2] On the tame fundamental groups of curves over algebraically closed fields of characteristic $>0$, in Galois groups and fundamental groups, Math. Sci. Res. Inst. Publ., 41, Cambridge Univ. Press, 2003, 47-105.

[Tam3] _ Finiteness of isomorphism classes of curves in positive characteristic with prescribed fundamental groups, J. Algebraic Geom., 13 (2004), 675-724.

[Tat] Tate, J. T., p-divisible groups, in Proc. Conf. Local Fields (Driebergen, 1966) Springer, 1967, 158-183.

[V] Vidal, I., Contributions à la cohomologie étale des schémas et des log-schémas, Thesis (Université de Paris-Sud, 2001). 